\title{
Interference in Cellular Networks: Sum of Lognormals Modeling
}

\author{
by \\ Sebastian S. Szyszkowicz \\ A thesis submitted to \\ The Faculty of Graduate Studies and Research \\ in partial fulfillment of the requirements \\ for the degree of \\ Master of Applied Science (Electrical Engineering) \\ Ottawa-Carleton Institute for Electrical and Computer Engineering \\ Department of Systems and Computer Engineering \\ Carleton University \\ Ottawa, Ontario, Canada, January 2007.
}

(ㄷ) Sebastian S. Szyszkowicz, 2007 


$\begin{array}{ll}\begin{array}{l}\text { Library and } \\ \text { Archives Canada }\end{array} & \begin{array}{l}\text { Bibliothèque et } \\ \text { Archives Canada }\end{array} \\ \begin{array}{l}\text { Published Heritage } \\ \text { Branch }\end{array} & \begin{array}{l}\text { Direction du } \\ \text { Patrimoine de l'édition }\end{array} \\ \begin{array}{l}\text { 395 Wellington Street } \\ \text { Ottawa ON K1A ON4 }\end{array} & \begin{array}{l}\text { 395, rue Wellington } \\ \text { Ottawa ON K1A ON4 } \\ \text { Canada }\end{array}\end{array}$

Your file Votre référence ISBN: 978-0-494-23350-4 Our file Notre référence ISBN: 978-0-494-23350-4

NOTICE:

The author has granted a nonexclusive license allowing Library and Archives Canada to reproduce, publish, archive, preserve, conserve, communicate to the public by telecommunication or on the Internet, loan, distribute and sell theses worldwide, for commercial or noncommercial purposes, in microform, paper, electronic and/or any other formats.

The author retains copyright ownership and moral rights in this thesis. Neither the thesis nor substantial extracts from it may be printed or otherwise reproduced without the author's permission.
AVIS:

L'auteur a accordé une licence non exclusive permettant à la Bibliothèque et Archives Canada de reproduire, publier, archiver, sauvegarder, conserver, transmettre au public par télécommunication ou par l'Internet, prêter, distribuer et vendre des thèses partout dans le monde, à des fins commerciales ou autres, sur support microforme, papier, électronique et/ou autres formats.

L'auteur conserve la propriété du droit d'auteur et des droits moraux qui protège cette thèse. $\mathrm{Ni}$ la thèse ni des extraits substantiels de celle-ci ne doivent être imprimés ou autrement reproduits sans son autorisation.
In compliance with the Canadian

Privacy Act some supporting forms may have been removed from this thesis.

While these forms may be included in the document page count, their removal does not represent any loss of content from the thesis.
Conformément à la loi canadienne sur la protection de la vie privée, quelques formulaires secondaires ont été enlevés de cette thèse.

Bien que ces formulaires aient inclus dans la pagination, il n'y aura aucun contenu manquant.

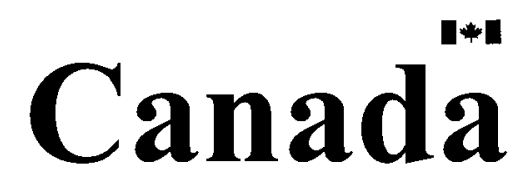




\begin{abstract}
We examine the existing methods for evaluating the distribution of the sum of lognormal random variables, focusing on closed-form results. We find that there are no results in literature that are both simple and accurate. We then derive a new closed-form expression for the lower tail of the distribution, and use it to construct a new method using a power-lognormal distribution. We apply both basic moment-matching and our new method the problem of the total interference power in a cellular system. For both methods, we derive equations that find the interference distribution essentially in closed form, using minimal numerical integration. We apply both methods to the uplink and downlink in systems with and without power control, for various cellular layouts, channel models and user activity probability. We compare distributions obtained by Monte-Carlo simulation directly with those obtained by our method, and find very good matches in many useful cases.
\end{abstract}




\section{Acknowledgements}

Çok teşekkür ederim and my warmest thanks to my supervisor, Dr. Halim Yanıkömeroğlu, for your academic and scientific guidance, and most of all for your humanity: your kind words of encouragement, your understanding, generosity, and patience. It has truly been a pleasure! My gratitude goes towards my examination committee, Dr. Mohamed El-Tanany, Chair, Dr. Florence Danilo-Lemoine, Dr. Fei Richard Yu, and my external examiner from the University of Ottawa, Dr. Abbas Yongaçoğlu. Many thanks also go to Dr. David D. Falconer, Dr. Eman Fituri and Dr. Shalini Periyalwar at Carleton University, and Dr. John Thompson at the University of Edinburg, UK, for their help and suggestions in the materials that went towards this thesis. I also want to thank all my colleagues, Ahmed Abdelsalam, Abdulkareem Adinoyi, Saad Al-Ahmadi, Muhammad Al-Juaid, Füruzan Atay, Akram Bin Sediq, Feroz Bokhari, Yijia Richard Fan, Youssouf Mouhamedou, Keivan Navaie, Mahmudur Rahman, and Mohamed Rashad for your help, kind words and fruitful discussions, for the soccer matches, and the good times at lunches and conferences. To good friends, Daniel, Jacek, Janek, Łukasz, Mark, Martin, Masoud, Michał and all the rest for the great times, the chess, the music, the tennis, the jokes, the deep discussions, the support, the fellowship, and for understanding that I cannot see you nearly as often as I would like. You guys are gold! Most importantly, I want to thank my parents Barbara and Mieczysław for the miracle of life and for the even greater miracle of raising me. Everything that is good is thanks to you. Everything that is bad is my fault alone and is left for me to fix on my own. I also want to thank you, who are both Ph.D.'s in Mathematics, for instilling in me the love of Mathematics for its own sake, which was the most practical thing you could do for my career. Finally, I want to thank you, my wonderful little sister Joanna Katarzyna Dobrochna for all the joy and laughter that you bring me, for cheering me up every time I was down and for understanding when your brother was working late in the lab. I love you so much!

iv 
This work was supported in part by Wireless Technology Labs, Nortel Networks, Ottawa, Canada, by a PGS M award from the Natural Sciences and Engineering Research Council (NSERC) of Canada, and by Carleton University, Canada. 
- Soli Deo Gloria - 


\section{Table of Contents}

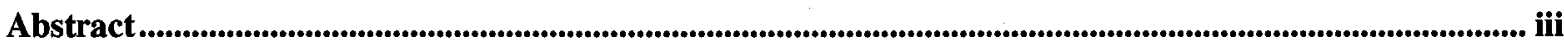

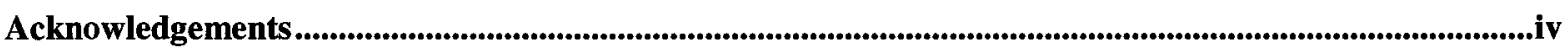

Table of Contents

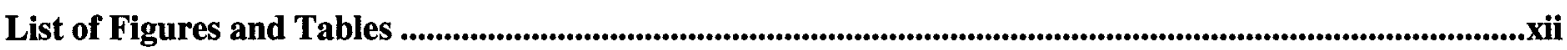

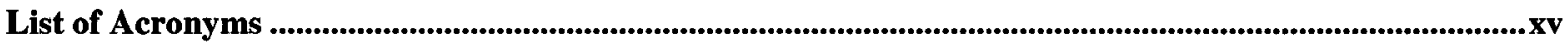

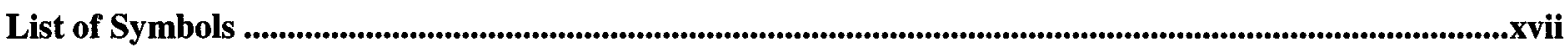

Chapter 1 Introduction: Total Interference in a Cellular Network.....................................................................1

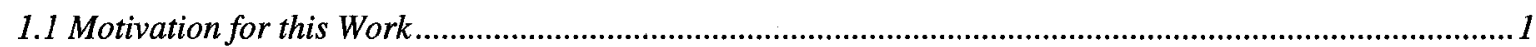

1.1.1 Some Previous Analytical Work on Interference ..................................................................2

1.1.2 Purpose and Motivation for a Closed-Form Solution ...............................................................

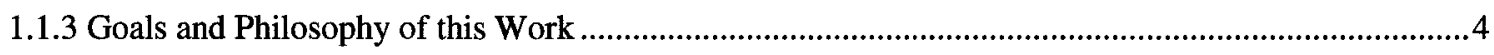

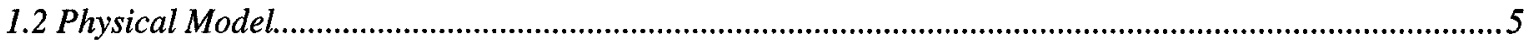

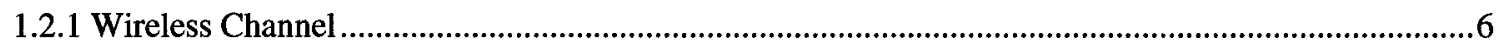

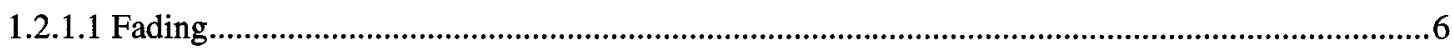

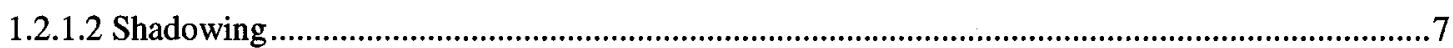

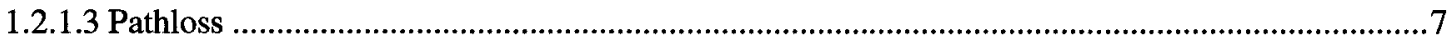

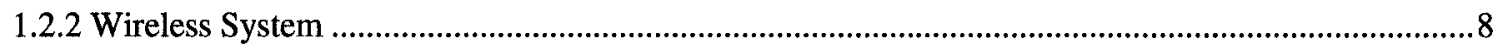

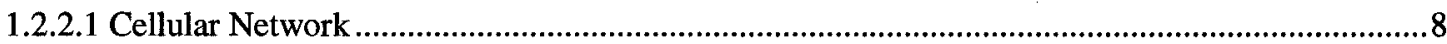

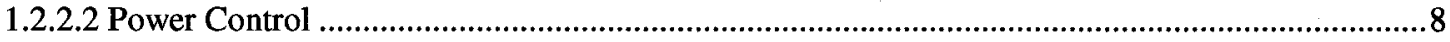

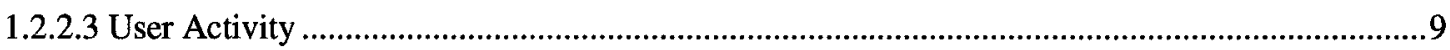

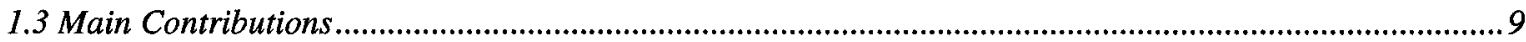

Chapter 2 Known Methods for Approximating the Distribution of the Sum of Lognormal Random

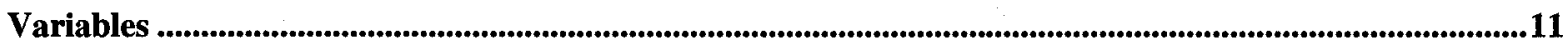

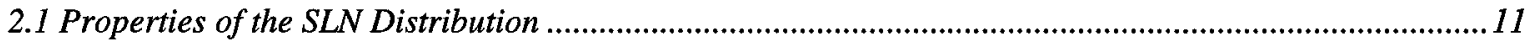

vii 
2.1.1 Definition

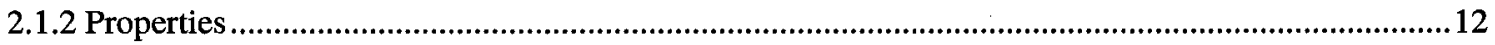

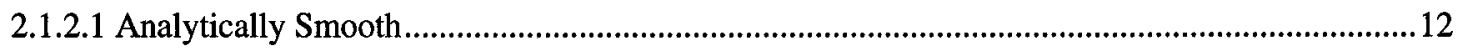

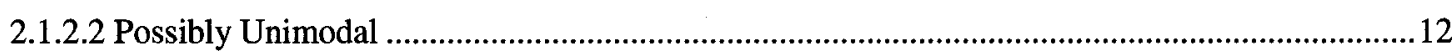

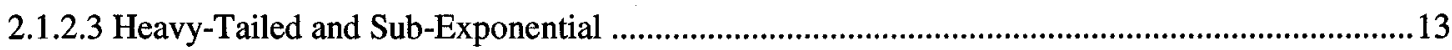

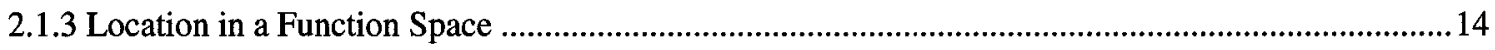

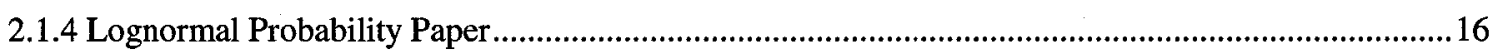

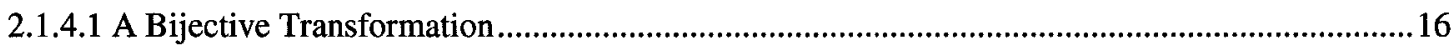

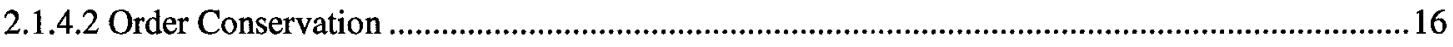

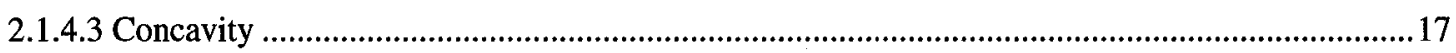

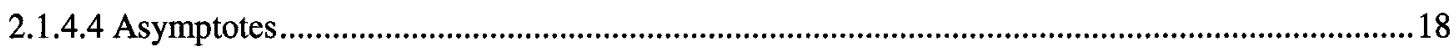

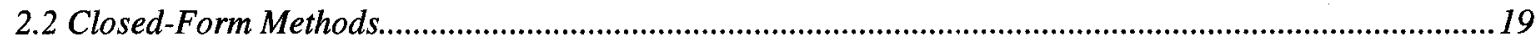

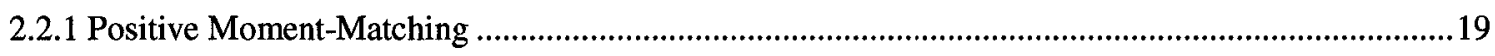

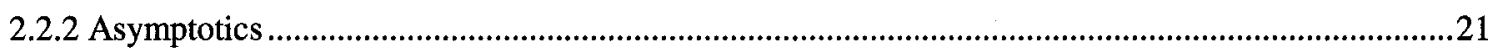

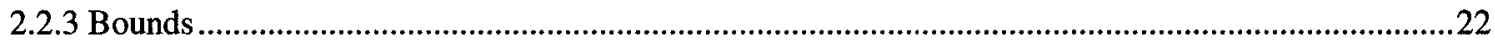

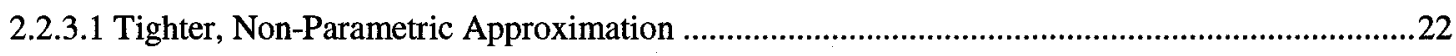

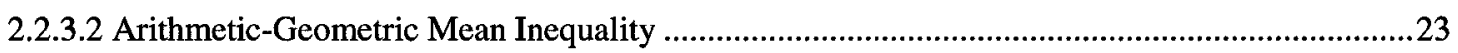

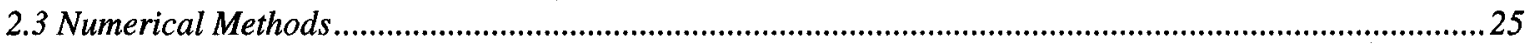

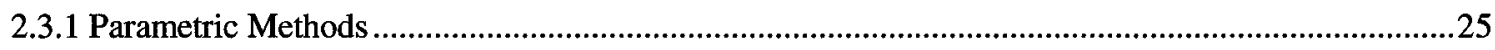

2.3.1.1 Logarithmic Moment-Matching: Schwartz - Yeh............................................................25

2.3.1.2 Log-Shifted Gamma Function: Le-Ngoc - Lam ........................................................26

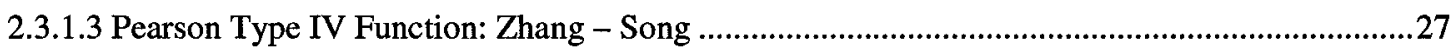

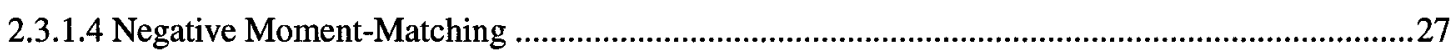

2.3.1.5 Flexible Lognormal Approximation: Wu - Mehta - Zhang..............................................22

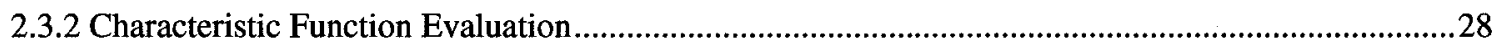

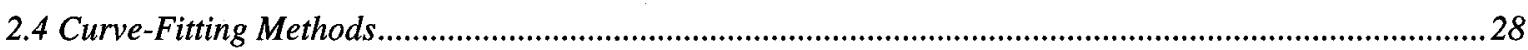

2.4.1 Minimax Lognormal Fitting: Beaulieu - Xie ......................................................................29

2.4.2 Three-Parameter Function: Beaulieu - Rajwani ..........................................................29

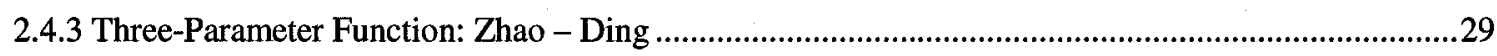

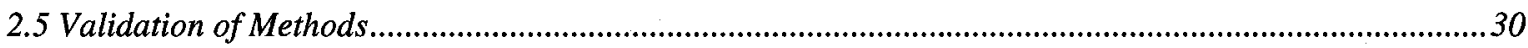

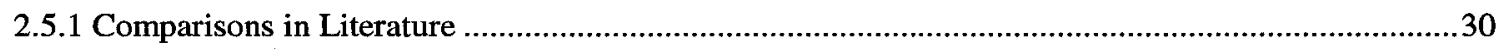

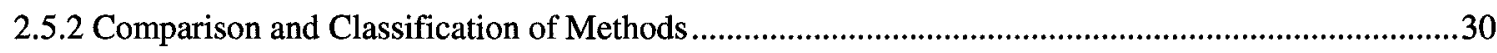

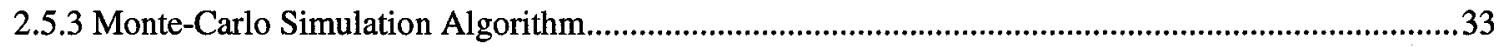

viii 
Chapter 3 Towards a Good, Simple, Closed-Form Approximation of the Distribution of the Sum of

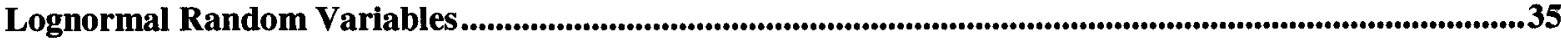

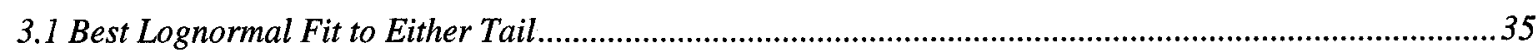

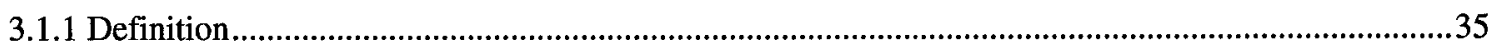

3.1.2 Best Lognormal Fit in the Linear Domain ........................................................................................36

3.1.3 Lower Tail (for Independent Summands) ......................................................................................38

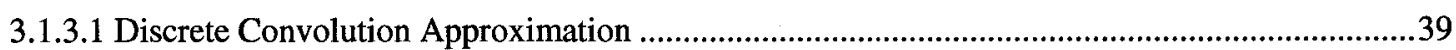

3.1.3.2 Scaled Lognormal Approximation ................................................................................................4

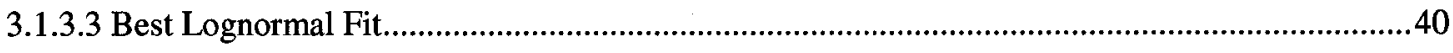

3.1.3.4 Difficulties in Approximating the Lower Tail.......................................................................... 41

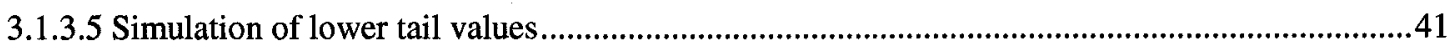

3.1.3.6 Comparison with Simulations and Recent Methods..................................................................4

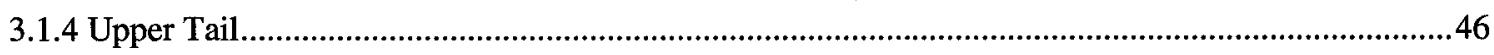

3.2 Potential Functions for Approximating the SLN Distribution ....................................................................46

3.2.1 Inadequacy of Existing Functions in the Tails.............................................................................46

3.2.1.1 Log-Shifted Gamma Function: Le-Ngoc - Lam (LL) ............................................................ 47

3.2.1.2 Three-Parameter Function: Beaulieu - Rajwani (BR)............................................................... 48

3.2.1.3 Three-Parameter Function: Zhao - Ding (ZD) ..........................................................................49

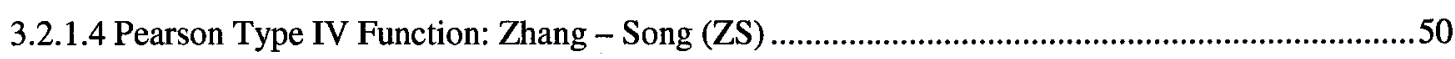

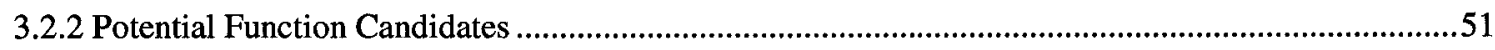

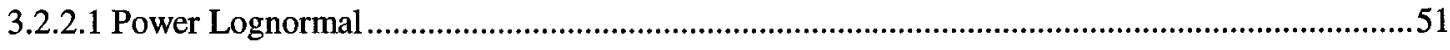

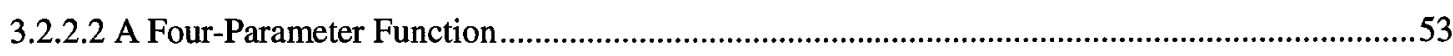

3.3 Obtaining the Parameters of the Approximating Functions ......................................................................54

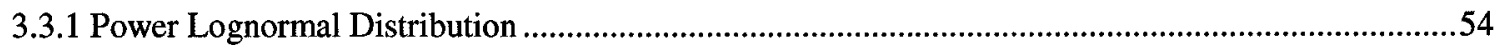

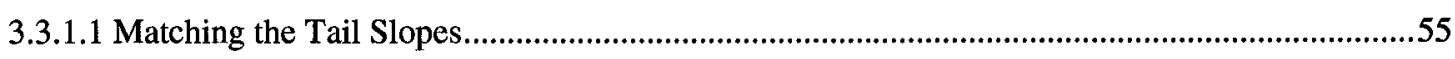

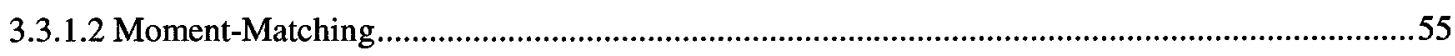

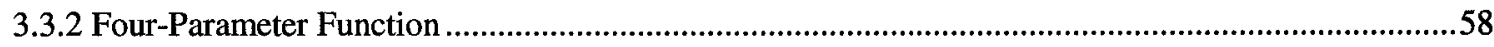

3.3.3 Evaluation of Closed-Form Methods against Simulations..............................................................5

Chapter 4 Statistical Analysis of Total Interference Power ...............................................................................................62

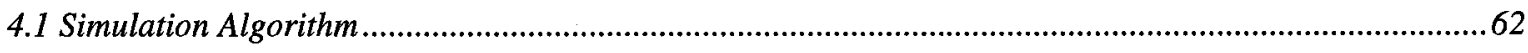

4.2 Distribution of the Interference Power from One Interferer ......................................................................66

4.2.1 Separating the Pathloss from all Other Factors....................................................................................6

4.2.2 Statistical Moments of the Inverse Pathloss ...............................................................................67

ix 
4.2.4 The Special Case of Uplink with Power Control ........................................................................72

4.2.5 Bernoulli Lognormal Approximation to Single Interferer Power Distribution..................................72

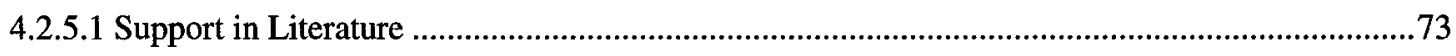

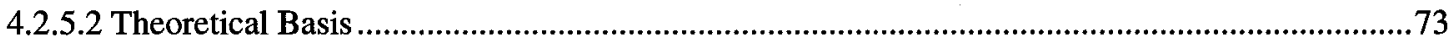

4.2.5.3 Fitting the Distribution by Moment-Matching ................................................................ 74

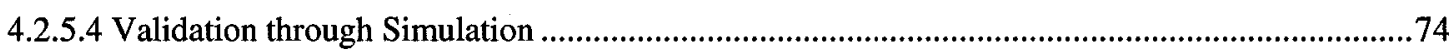

4.3 Statistical Moments of Total Interference Power ...........................................................................79

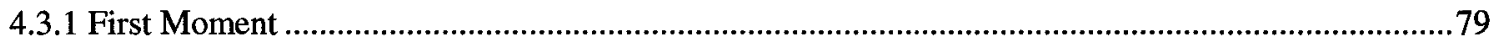

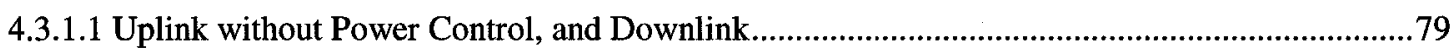

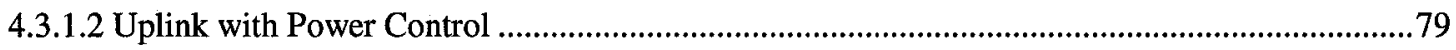

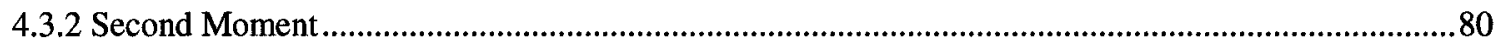

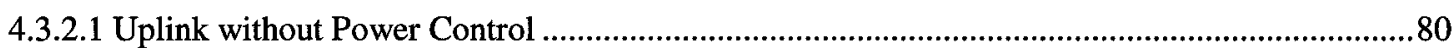

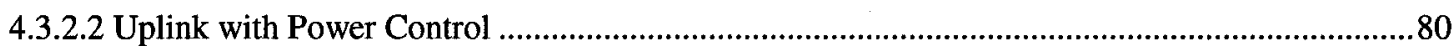

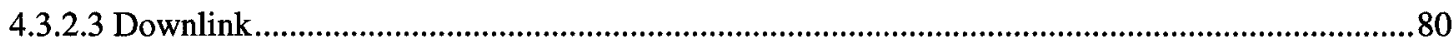

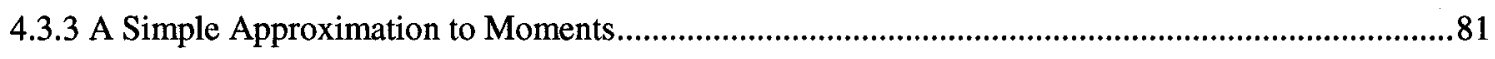

4.4 Modeling the Total Interference Power as Bernoulli Lognormal ........................................................82

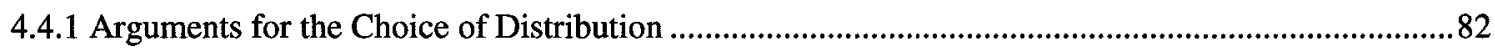

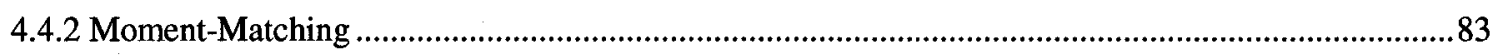

4.4.3 Simple Approximations to the Distribution Parameters ...........................................................83

4.5 Modeling the Total Interference Power as Bernoulli Power Lognormal..............................................84

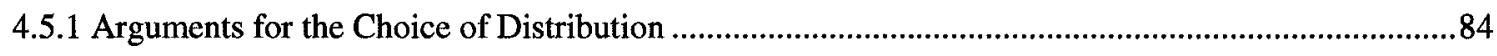

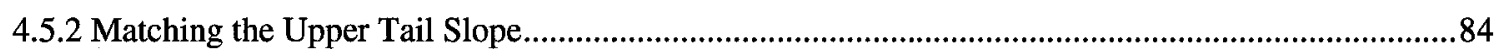

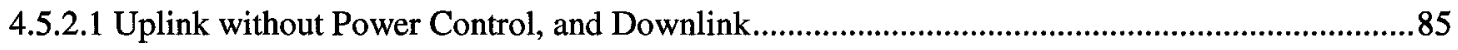

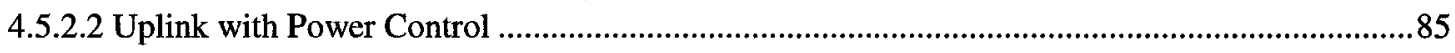

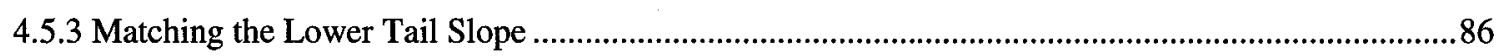

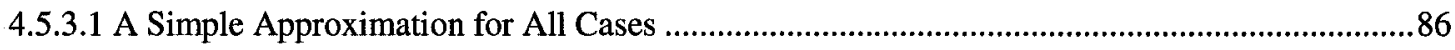

4.5.3.2 Uplink without Power Control, and Downlink............................................................86

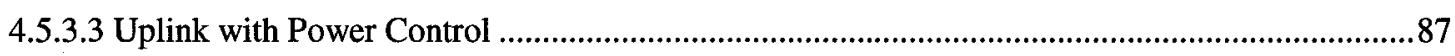

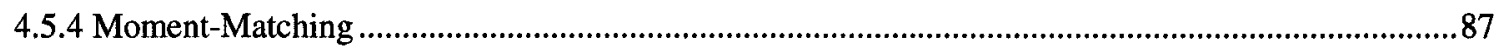

4.5.4.1 Uplink without Power Control, and Downlink............................................................8

4.5.4.2 Uplink with Power Control .................................................................................................88

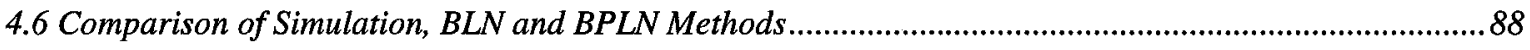

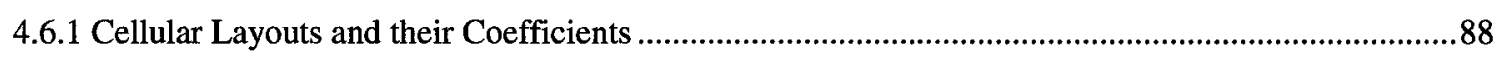

4.6.2 Simulated and Analytical Parameters and Graphs .................................................................91 


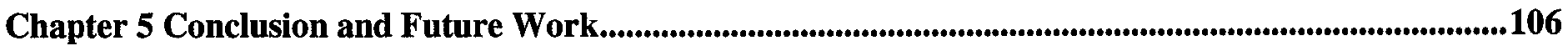

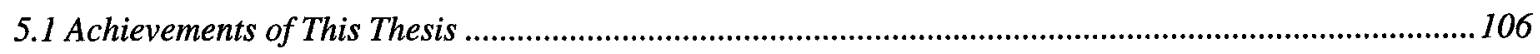

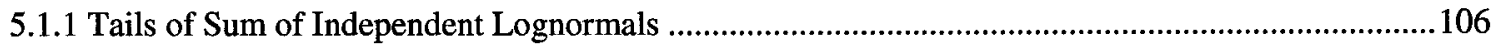

5.1.2 Simple Expressions for Moments of Total Interference Power ................................................. 106

5.1.3 Approximate Interference Distributions: Two Methods ...........................................................107

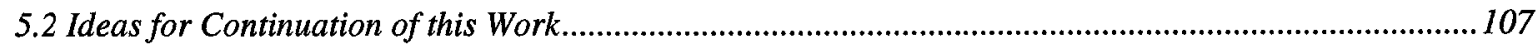

5.2.1 Improving the SLN and Interference Approximations .......................................................... 107

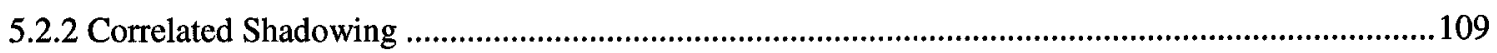

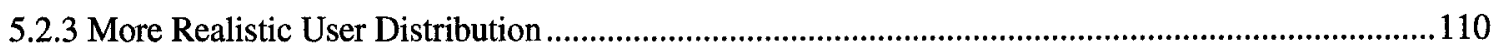

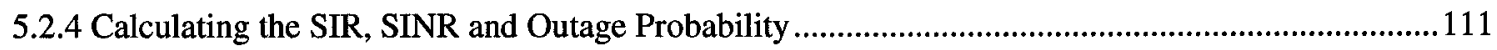

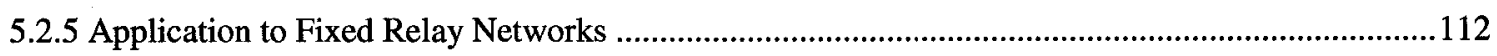

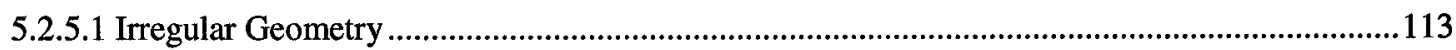

5.2.5.2 Lower Probability of Activity per Sub-cell ................................................................113

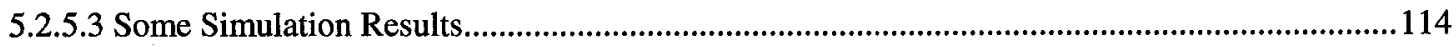

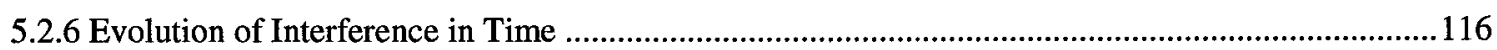

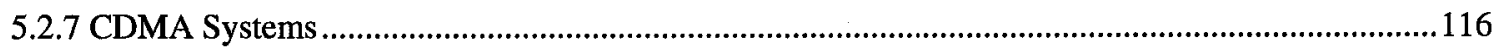

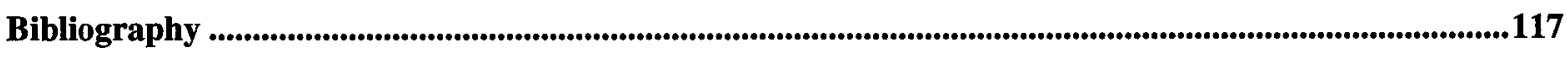


List of Figures and Tables

Figure 2-1 Locus of successive convolutions of LN pdf's in a function space. Taken from [18] 15

Figure 2-2 $c d f$ on LN paper of the i.i.d. SLN with $\sigma=12 \mathrm{~dB}$. Taken from [35] . .18

Table 2-1 Summary of properties of SLN methods .32

Figure 2-3 Comparison of SLN methods by complexity and precision. 33

Figure 2-4 Algorithm that simulates the SLN $c d f$. .34

Figure 3-1 Algorithm that efficiently simulates the far lower tail of the SLN $c d f$. .042

Table 3-1 Simulation parameters for SLN $c d f$ 's in the lower tail 44

Figure 3-2 The $c d f$ of the sum of two i.i.d. LN RV's $-\mathrm{A}: \sigma=6 \mathrm{~dB}$ and $\mathrm{B}: \sigma=12 \mathrm{~dB}$ . .44

Figure 3-3 The cdf of the sum of lognormals with different variances. .45

Figure 3-4 The $c d f$ of the sum of lognormals with different means .045

Table 3-2 Analytical and numerical calculations of moments of the PLN distribution when the power is $t=2$, with differences underlined. .57

Table 3-3 Simulation parameters for sums of lognormals. .59

Figure 3-5 The $c d f$ of the sum of 6 i.i.d. lognormals, and closed-form methods .66

Figure 3-6 The $c d f$ of the sum of 30 i.i.d. lognormals, and closed-form methods. 60

Figure 3-7 The $c d f$ of the sum of 12 lognormals with different means, and closed-form methods. .61

Figure 3-8 The cdf of the sum of 36 lognormals with different means and variances, and closed-form methods. 
Figure 4-1 Normalised MU and FS positions in a complex plane. .63

Figure 4-2 Algorithm for simulating interference in cellular systems. 64

Figure 4-3 Algorithm to calculate $I_{i}$ .65

Figure 4-4 Three tiers of cells with five different cell positions (classes A-E). .67

Table 4-1 $\kappa_{i}(\beta)$ function tabulated for all cell classes and pathloss exponents. 68

Table 4-2 $\kappa_{i}(2 \beta)$ function tabulated for all cell classes and pathloss exponents. .68

Table 4-3 $\tilde{\kappa}_{i}(\beta)$ function tabulated for all cell classes and pathloss exponents. .71

Table 4-4 $\tilde{\kappa}_{i}(2 \beta)$ function tabulated for all cell classes and pathloss exponents. .71

Table 4-5 Simulation parameters for single interferer power. .075

Figure 4-5 Single nearby interferer, Rayleigh fading, without power control. .76

Figure 4-6 Single nearby interferer, no fading, downlink with power control. .76

Figure 4-7 Single nearby interferer, Rician fading, uplink with power control. .77

Figure 4-8 Single distant interferer, no fading, without power control. .77

Figure 4-9 Single distant interferer, Rician fading, downlink with power control .078

Figure 4-10 Single distant interferer, Rayleigh fading, uplink with power control. $\mathbf{7 8}$

Table 4-6 Geometry parameters for various cellular layouts. .89

Table 4-7 Simulation and analytical parameters for BLN and BPLN methods. . .92

xiii 
Figure 4-11 Interference in three-tier cellular network with variable activity in the downlink without power control

Figure 4-12 Comparison of interference from one and three tiers in the downlink with power control....96

Figure 4-13 Effect of varying the shadowing parameter on interference from first tier in the uplink without power control.

Figure 4-14 Interference in a reuse 7 cellular network in the uplink without power control. . .98

Figure 4-15 Interference in reuse 3 cellular network with variable activity level in the downlink with power control.

Figure 4-16 Interference from first tier with Rayleigh, Rician and no fading in the downlink with power control.

Figure 4-17 Interference from first tier while varying propagation exponent, shadowing and fading in the uplink with power control.

Figure 4-18 Interference in reuse 3 cellular network with variable activity level in the uplink with power control 102

Figure 4-19 Interference from a random layout of cells in the uplink with power control .103

Figure 5-1 Interfering cells (in gray) in a two-hop fixed relay network. .112

Table 5-1 Geometry parameters for three tiers of sub-cells in a two-hop fixed relay network. .113

Table 5-2 Simulation and analytical parameters for a two-hop fixed relay network. .114

Figure 5-2 Interference from a fixed relay network in the downlink with power control. .115 


\section{List of Acronyms}

AG

blf

BLN

BPLN

BR

BS

BX

$c d f$

CDMA

$c f$

DL

DMU

FH-SS

FS

FW

h.t.

i.i.d.

i.n.i.d.

IFS

IMU

LL

LN

LSG

MU

OFDMA

PC

$p d f$

PLN

RS

RV

s.e.
Arithmetic-Geometric mean inequality method [46]

Best lognormal fit

Bernoulli (times) Lognormal

Bernoulli (times) Power Lognormal

Beaulieu - Rajwani (distribution function) [27]

Base Station

Beaulieu - Xie (method for analysing the SLN problem) [35]

Cumulative distribution function

Code-Division Multiple Access

Characteristic Function (of a RV)

Downlink

Desired Mobile User (MU under analysis)

Frequency Hopping (Spread Spectrum technique)

Fixed Station (i.e., a BS or RS)

Fenton - Wilkinson (method for analysing the SLN problem) [1]

Heavy-Tailed (property of distributions)

Independent, identically distributed (random variables)

Independent, but not necessarily identically distributed (random variables)

Interfering Fixed Station

Interfering Mobile User (MU whose link causes interference to the DMU)

Le-Ngoc - Lam (distribution function) [27]

Lognormal

Log-Shifted Gamma (Distribution function used in the LL method) [27]

Mobile User/Unit

Orthogonal Frequency-Division Multiple Access

Power Control

Probability density function

Power Lognormal

(fixed) Relay Station

Random Variable

Sub-Exponential (property of distributions)

$\mathrm{xv}$ 
SINR

SIR

SLN

SY

$\mathrm{UL}$

WMZ

$\mathrm{ZD}$

$\mathrm{ZS}$
Signal to Interference and Noise (Power) Ratio

Signal to Interference (Power) Ratio

Sum of Lognormal (Random Variables)

Schwartz and Yeh (method for analysing the SLN problem) [37],[47]

Uplink

Wu-Mehta-Zhang (method for analysing the SLN problem) [35]

Zhao - Ding (distribution function) [28]

Zhang-Song (distribution function) [28] 


\section{List of Symbols}

$\#\{\subseteq\} \quad$ Cardinality of set $\mathfrak{S}$

$a_{I}$

$\mu$-parameter of the BLN approximation to $F_{I}(x)$

$A_{n}, A_{D L} \tilde{A}_{n} \quad A$-coefficients describe the pathloss statistics for a given cellular layout.

$b_{I} \quad \sigma$-parameter of the BLN approximation to $F_{I}(x)$

$B_{n} \quad n^{\text {th }}$ statistical moment of any $Q_{i}$

$\tilde{B}_{n} \quad n^{\text {th }}$ statistical moment of any $Q_{i} / L_{i}$

$C_{0} \quad$ Cell of interest

$C_{l} \quad$ A nearest interfering cell

$C_{i} \quad$ Interfering cell

$E[$ Q] $\quad$ Expected value of RV $\mathscr{X}$

$F_{\mathscr{X}}(x) \quad c d f$ of $\mathrm{RV} \mathscr{X}$

$f_{\mathscr{X}}(x) \quad p d f$ of $\mathrm{RV} \mathscr{X}$

$\tilde{F}(x) \quad F(x)$ transformed under $T$

$\mathrm{RV}$ : Total received interference power

$i, j \quad$ Index of interfering cell or lognormal summand

$I_{i} \quad \mathrm{RV}:$ Received interference power coming from cell $C_{i}$

$K \quad$ Rician fading $K$-factor in linear scale: 0 - Rayleigh fading, $\infty$ - no fading

$k_{D L} \quad$ Downlink option indicator: 0- UL, $1-\mathrm{DL}$

$k_{P C} \quad$ Power control option indicator: $0-$ no PC, $1-$ PC

$l \quad+\infty$ or $-\infty$ : indicates the upper and lower tail respectively on lognormal paper

$L_{i} \quad \mathrm{RV}$ : Pathloss between $\mathrm{FS}_{i}$ and $\mathrm{MU}_{i}$

$m \quad \mu$-parameter of the PLN distribution

$m_{I} \quad \mu$-parameter of the BPLN approximation to $F_{I}(x)$

$N \quad$ Number of interfering cells or number of terms in a sum of lognormals

$n \quad n^{\text {th }}$ statistical moment

$p \quad$ Probability of activity of an interferer from any given cell

$P[$ G $\quad$ Probability of event $\mathcal{E}$

$P_{i} \quad \mathrm{RV}:$ Normalised transmit power of interferer from cell $C_{i}$ due to power control;

xvii 
without power control: constant $=1$

$\mu_{L}$

$\mu_{U}$

Probability of activity of at least one interferer

$\mathrm{RV}$ : Product of all factors affecting the received power from cell $C_{i}$, except pathloss

RV: Inverse pathloss suffered by interfering signal from cell $C_{i}$

RV: Physical (normalised) distance from interferer in cell $C_{i}$

$\sigma$-parameter of the PLN distribution

$\sigma$-parameter of the BPLN approximation to $F_{I}(x)$

Transformation (mapping) from linear to lognormal probability paper

Power parameter of the PLN distribution

Power parameter of the BPLN approximation to $F_{I}(x)$

$\mathrm{RV}$ : sum of lognormal RV's $Y_{i}$

Cartesian coordinates of $\mathrm{FS}_{i}$ (equivalently, the centre of cell $C_{i}$ )

$\mathrm{RV}$ : lognormal, with parameters $\mu_{i}, \sigma_{i}$

Auxiliary term in calculating the $B P L N$ parameters to approximate $F_{I}(x)$

RV: Instantaneous fading envelope amplitude in linear scale of signal from cell $C_{i}$

Pathloss exponent

Incomplete (lower) gamma function

Gamma function

Standard normal $c d f$

RV: Lognormal-distributed shadowing in linear scale suffered by interfering signal from cell $C_{i}$

Pathloss statistics for received power from cell $C_{i}$

Pathloss statistics for received power from cell $C_{i}$ in the case of uplink, power control Constant factor multiplying the blf to the lower tail of the SLN distribution Constant factor multiplying the blf to the upper tail of the SLN distribution Constant $=\ln 10 / 10 \approx 0.23$, used to convert between $\mathrm{dB}$ and natural units Integral used to find the moments of the PLN distribution $\mu$-parameter of lognormal RV $Y_{i}$ $\mu$-parameter of the blf to the lower tail of the SLN distribution $\mu$-parameter of blf to the upper tail of the SLN distribution Constant $=0.915$, radius of circle that approximates well a unit hexagon 
$\rho_{i j} \quad$ Correlation coefficient between two Gaussian RV's with indices $i, j$

$\sigma \quad$ Shadowing variance in natural units

$\sigma_{e} \quad$ Power control error lognormal variance in natural units

$\sigma_{i} \quad \sigma$-parameter of lognormal RV $Y_{i}$

$\sigma_{L} \quad \sigma$-parameter of the blf to the lower tail of the SLN distribution

$\sigma_{U} \quad \sigma$-parameter of blf to the upper tail of the SLN distribution

$\chi_{i} \quad \mathrm{RV}:$ Bernoulli-distributed indicator of activity of interferer from cell $C_{i}$

Many other symbols are used locally within sub-sections, sometimes overriding the above definitions. Hopefully, this will always be clear from context. 


\section{Chapter 1 \\ Introduction: Total Interference in a Cellular Network}

\subsection{Motivation for this Work}

While the problem of analysis of interference in cellular systems is at least as old as the first systems themselves, the topic still attracts much interest. This is essentially because the problem is not really solved yet, though there exist many methods that solve particular problems, with varying levels of complexity (numerical or closed-form), and various levels of accuracy. There does not seem to be a well-established systematic method to analyse this class of problems, and several papers [1]-[16] work on very similar problems without there being much incremental knowledge. Indeed, these papers have a substantial portion of overlap, particularly when it concerns two essential mathematical problems: calculating the effect of the mobile user's (MU) random distribution in space, and evaluating the sum of lognormal (SLN) random variables (RV). In this thesis we propose to make a synthesis of the common points of the work in these papers. We do this first by creating a mathematical and simulation model that is sufficiently detailed to encompass many particular problems, but simple enough for analytical tractability. We then evaluate the existing methods for calculating the SLN distribution, and develop our own method based on a synthesis of several known results. Finally we apply this method to the interference distribution problem, and obtain simple expressions to calculate an approximation to this distribution as a function of the parameters of a particular problem. In particular, we can separate the geometry of the cellular layout (equivalently, the user distribution) from the rest of the problem, which means that some geometrical parameters can be calculated once and for all and tabulated for future use. We believe that the method we propose is flexible enough to 
accommodate many classical problems while retaining its simplicity, and also can be modified to solve new problems in emerging wireless systems.

The main underlying mathematical problem is the SLN distribution, which appears not only in wireless communications, but also in electronics [17], physics [18], optics [19], economics [20], and is of interest to statistical mathematicians [21], [22]. This versatility makes the problem even more important to solve.

Knowing the distribution of the interference in a system is the next best thing to actually knowing the interference at any given moment. Indeed, unless one uses adaptive channel mechanisms [1], [2] or cognitive radio techniques, one has to allocate scarce radio resources (spectrum, power) based on statistical knowledge. A precise knowledge of the interference cumulative distribution function $(c d f)$ is necessary for optimal design: if the interference is overestimated, radio resources are wasted in trying to avoid outage situations that almost never happen, while if it is underestimated, the quality of service suffers from too frequent outages. In past years, not only cellular, but many other wireless technologies have been emerging (WiFi, WiMax, Bluetooth, UWB, ...), and much research is currently being done in ad-hoc, mesh and sensor networks. All these often use shared, unlicensed bands. As so many wireless technologies come to share the same spectrum, the systems become interference-limited. While it is not the purpose of this thesis to explore the interference between such systems, simply understanding the statistical behaviour of interference becomes more important than ever.

\subsubsection{Some Previous Analytical Work on Interference}

In this section we touch on two previous papers that analyse interference, we show why they are important and also what is lacking in them. It is these lacks that we want to fill in this thesis. We borrow the mathematical models in order to formulate the problem in Section 1.2. 
Reference [3] is the work that is closest to ours and has inspired many of the points in this thesis. First of all, it is one of the rare papers that introduce the concept of geometrical coefficients, integrals that can be calculated independently of other system considerations, and tabulated for reference. Thus a particular cellular layout may be described by a collection of numbers. These can be substituted into closed-form expressions in order to obtain the interference statistics in a particular cellular system. A weakness of this paper is that many of the stochastic quantities have been replaced by their expected value, which is an over-simplification.

Reference [1] uses a numerical method by Schwartz and Yeh (SY) (see Section 2.3.1.1). The disadvantage of this paper is its heavy use of numerical integration at all stages of the process. We intend to show in our work that much of it is unnecessary, and that a tractable solution exists.

Both papers study interference in the uplink (UL). In [3], the downlink (DL) is also examined, but there is little analysis done and the method becomes mostly numerical. We intend to show that we may find closed-form expressions not only for the UL, but for the DL as well. The DL, though more challenging analytically, must be well understood because of the heavy traffic asymmetry leaning towards the DL in future systems [23]. The greatest weakness of both papers is the lack of a direct comparison between simulation results and the theoretically derived distributions. In fact, it is rare to find a comparison between a simulated and calculated distribution curve of the interference statistics. We will always support our theoretical results with Monte-Carlo simulations in this thesis.

\subsubsection{Purpose and Motivation for a Closed-Form Solution}

Reference [24] suggests that knowing the statistics of the interference coming from just one interferer is not enough, and that knowledge of the sum interference power is necessary for accurate interference modeling. We consider a cellular system with known layout, a propagation 
model, user behaviour statistics, and potentially a power control (PC) mechanism. We can simulate interference to a particular desired mobile user (DMU) in such a system according to the model described in Section 1.2, using Monte-Carlo Simulation. This thesis has one main goal: to reproduce the Monte-Carlo interference $c d f$ as closely as possible using, as much as possible, only analytical, closed-form expressions, with minimal numerical integration. This is useful for the following reasons:

1. Monte-Carlo simulations can be very time-consuming, while numerical integrations, if well-behaved, are substantially faster. Also, analytical expressions programmed into a computer execute almost instantaneously.

2. A simple closed-form solution to a problem is always desirable: we try to aim for expressions that can be evaluated using a scientific calculator and that could be included in textbooks on basic wireless communications. Such a method is much more likely to gain popularity than one which takes a significant time to learn and implement.

3. A closed-form method to a simple problem forms a basis for further analysis of more complex ones, such as those in emerging next-generation wireless systems.

4. Closed-from expressions are useful in order to understand the exact effect of every system parameter on the final performance of the system. We can for example take derivatives with respect to the parameters and see how sensitive the system is to them.

\subsubsection{Goals and Philosophy of this Work}

Throughout our work, we have kept in mind the following criteria that our method should fulfill:

1. As much as possible, obtain closed-form expressions.

2. As much as possible, use only standard functions found on a scientific calculator. 
3. Obtain analytical $c d f$ curves that are close (within $1 \mathrm{~dB}$ ) to the simulated interference $c d f$ at all points of the curve.

4. As much as possible, simplify the expressions: if removing a term does not increase the error between the analytical and simulated curves by a significant percentage, then that term should be omitted.

5. As much as possible, avoid numerical methods.

6. When numerical integration is necessary, let the integral be separable as much as possible from the closed-form part.

7. When numerical integration is necessary, let it be easily converging using Riemann sums with a moderate number of steps.

8. As much as possible, let the numerical integrations be general enough that they can be tabulated and later plugged into closed-form expressions.

9. The final distribution should be an analytical function with a fixed form and described by a few parameters. This way, only a few real numbers are sufficient to entirely describe the curve. Piecewise, implicit, or recursive solutions are undesirable.

\subsection{Physical Model}

We would like to propose a system model that is as general as possible, while retaining enough simplicity for analytical tractability. Our model is based closely on those used in [1]-[3], which apply to flat-fading channels, and is thus well suited for frequency-hopping spread-spectrum (FHSS), as well as orthogonal frequency division multiple access (OFDMA). The wireless system is divided into cells, each served by a base station (BS). However, we may also study a wireless system with fixed relays [2], where each relay station (RS) defines a sub-cell around itself, and 
the MU connects to the nearest RS or BS. In order to encompass both conventional and relayed networks, we call a BS or RS by the common name of fixed station (FS), the exact nature of which is irrelevant to the analysis at this level. We study systems where there can be at most one other user per FS using the same frequency channel at the same time, thus one interferer per FS. The various interfering signals can be considered incoherent amongst themselves, thus the total interference power received can be written as:

$$
I=\sum_{i=1}^{N} I_{i}=\sum_{i=1}^{N} R_{i} \zeta_{i} \chi_{i} \alpha_{i}^{2} P_{i}
$$

Here there are $N$ potential interferers (equivalently, $N$ FS's), and $I_{i}$ is the interference due to FS $i$. In the UL, there are $N$ interfering mobile users (IMU), each transmitting to their own FS a signal that is also received as interference by the desired FS. In the DL, it is the interfering fixed stations (IFS) that transmit to their own MU's a signal that is also received as interference by the DMU. $R_{i}$ is the average path gain (inverse of pathloss) to the interferer. $\zeta_{i}$ and $\alpha_{i}^{2}$ represent shadowing and fading respectively between the interferer $i$ and the receiver. $\chi_{i}$ is the Bernoulli-distributed indicator function that is unity when there is an active user in interfering cell $C_{i}$ on the channel under consideration, otherwise zero. $P_{i}$ is the (normalised) transmit power coming from the interferer in cell $\mathrm{C}_{i}$.

\subsubsection{Wireless Channel}

\subsubsection{Fading}

We consider a system such as OFDMA or FH-SS where flat fading occurs over any single channel. There are several fading models in existence. We consider a Rician model with parameter $K$. When $K=0$, the model becomes Rayleigh, and the fading power factor is exponentially distributed with parameter $\lambda=1$ such that $E\left[\alpha_{i}^{2}\right]=1$. When $K=\infty$, there is no 
fading in the model. In general, for Rician fading, such that $E\left[\alpha_{i}^{2}\right]=1$, we have the following probability density function $(p d f)[25]$ :

$$
f_{\alpha_{i}}(x)=2 x(K+1) \exp \left(-x^{2}(K+1)-K\right) I_{0}\left(2 x \sqrt{K^{2}+K}\right), x>0,
$$

where $I_{0}$ is the modified Bessel function of the first kind. The fading power multiplied by $2(K+1)$ follows a non-central $\chi^{2}$ distribution with 2 degrees of freedom and non-centrality parameter $2 K$ :

$$
\begin{gathered}
\frac{\partial}{\partial x} P\left(\alpha_{i}^{2} 2(K+1)<x\right)=\frac{1}{2 \sqrt{2 x(K+1)}} f_{\alpha_{i}}\left(\sqrt{\frac{x}{2(K+1)}}\right) \\
=\frac{1}{2} \exp \left(-\frac{1}{2} x-K\right) I_{0}(\sqrt{2 x K}), x>0 .
\end{gathered}
$$

\subsubsection{Shadowing}

The interfering signal also suffers long-term shadowing, which follows a lognormal (LN) law, with $\sigma(\mathrm{dB})=6$ to $12 \mathrm{~dB}$ spread. All paths are assumed to be independently faded and shadowed. In this thesis, we use the natural base for LN RV's. Thus we convert from decibel units (typically used for shadowing) to natural units by multiplying by a factor of $\lambda=\ln (10) / 10 \approx 0.23$. In natural units, $\sigma=\lambda \sigma(\mathrm{dB})$.

\subsubsection{Pathloss}

The average distance-dependent pathloss model is [2]:

$$
\frac{1}{R_{i}}=r_{0}^{-\beta}\left(\frac{4 \pi r_{0} f}{c}\right)^{2} r_{i}^{\beta},
$$

where $r_{0}$ is the close-in reference distance, $f$ the carrier frequency, $c$ the speed of light, $\beta$ is the propagation exponent and $r_{i}$ is the distance between the transmitter and receiver. Without loss of generality, we can ignore the constant factor, in which case $R_{i}$ is equal to $r_{i}^{-\beta}$, where $r_{i}$ is the 
physical normalised distance between the interferer $i$ and the receiver. In general, $R_{i}$ 's have different distributions, and are not independent for different $i$ when considering the DL.

\subsubsection{Wireless System}

We use the indicator $k_{D L}$ to specify whether we are looking at the UL or the DL. In the UL $\left(k_{D L}=\right.$ $0)$, the desired FS can receive interference signals from MU's in other cells. In the DL $\left(k_{D L}=1\right)$, it is the MU under analysis, located in $C_{0}$, that receives interference from other FS's, which are transmitting to their own MU's. The marginal distribution of the interference power from a given cell $C_{i}$ is identical for UL and DL. However, only for the UL are the pathlosses independent for each interferer.

\subsubsection{Cellular Network}

We assume that all the cells are identical regular hexagons of unit side length, each with its FS in the center. A MU is assumed to be connected to its nearest FS, i.e., it lies in the corresponding hexagon. The position of the MU is assumed random, uniformly distributed over the cell. We call the cell under consideration $C_{0}$, and all potentially interfering cells $C_{i}$.

Although we assume all cells to be hexagons, there is nothing in the analysis that prevents us from considering, for example, square cells in a Manhattan-style grid. The integrals and distributions that involve the cell geometry would need to be modified.

\subsubsection{Power Control}

We use the indicator $k_{P C}$ to specify whether we are looking at a system with or without PC. Without loss of generality, we assume that, without $\mathrm{PC}\left(k_{P C}=0\right)$, all interferers are transmitting at unit power. Under PC $\left(k_{P C}=1\right)$, the power $P_{i}$ transmitted by the $i^{\text {th }}$ interferer has identical statistics for UL and DL. The PC mechanism is such that it attempts to compensate for shadowing 
and pathloss is such a way that a FS receives the same power from each of its MU's, within some error margin [1], [3]. The transmit power $P_{i}$ depends on the pathloss between the IMU and its IFS, the LN shadowing with spread $\sigma$ and the PC LN error with spread $\sigma_{e}(\mathrm{~dB})=0$ to $1 \mathrm{~dB}$. The PC mechanism is assumed slow: it does not compensate for signal fading.

\subsubsection{User Activity}

We assume that each MU within a cell uses an orthogonal channel. Consequently, there is only intercellular interference, and there can be at most one interferer in each cell $C_{i}$. Thus, the presence of interference from a particular cell $C_{i}$ can be modeled by a Bernoulli RV with parameter $p$ (collision probability), which is a function of loading, number of channels, channel reuse, DTX [1], etc. It is an appropriate model for any channel in a FH-SS or OFDMA system, where there may or may not be activity on each given carrier, and the frequencies are assigned orthogonally within each cell.

We assume that the Bernoulli RV that models the activity is independent of all other quantities. In reality, the activity is generally dependent on the interference at a given point in time, because a new user may be admitted to the channel based on the activity level of other users on that channel. However, we do not model this effect here.

\subsection{Main Contributions}

The main analytical contributions of this thesis are:

1. A closed-form expression for the lower tail asymptote to the SLN distribution. It is given by (3.9), (3.11), (3.12).

2. Building on ideas from [3], and based on the Fenton-Wilkinson (FW) [26] method for SLN analysis, a closed-form expression (4.39), (4.40), (4.34), (4.35), for the interference in the 
model described in Section 1.2. Our expression separates the cellular layout from other parameters, and we provide a lookup table from which the parameters describing the layout can be obtained. This method performs well in the body and tail, for lower probabilities of activity.

3. Using the two previous points, a novel method for solving the SLN problem using a powerlognormal (PLN) distribution. This method is described by (3.35), (3.48)-(3.51). We apply this method to obtain simple forms for the distribution parameters (4.41)-(4.46), (4.52), (4.54). Only one simple numerical integral is required (3.50). This method performs well over the entire range of values for high activity levels.

Furthermore, this thesis contains:

4. A survey of various properties of the SLN distribution.

5. A thorough survey of methods to solve the SLN problem, including the most recent findings (end of 2006).

6. A formal framework to study the tails of the SLN distribution, and a study of the tails of several recent SLN methods [27]-[30].

7. A four-parameter function that approximates the SLN $c d f$ with reasonable accuracy over the entire range of values, and whose parameters are found in closed-form.

8. Several ideas for the continuation of this work with suggested approaches. 


\section{Chapter 2 \\ Known Methods for Approximating the Distribution of the Sum of Lognormal Random Variables}

The fundamental mathematical problem that lies behind the interference analysis is calculating the distribution of the SLN. Although the SLN distribution is not known in closed form, there are nevertheless many things that we do know about it. We give an overview of the properties of the SLN distribution, and of the methods used in literature to approximate it.

\subsection{Properties of the SLN Distribution}

\subsubsection{Definition}

The problem can be mathematically stated as follows: Let

$$
X=\sum_{i=1}^{N} Y_{i}
$$

We assume in this thesis that the summands $Y_{i}$ are independent. The $p d f$ of each summand is LN:

$$
Y_{i} \sim f_{i}\left(x ; \mu_{i}, \sigma_{i}\right)=\frac{1}{\sqrt{2 \pi} x \sigma_{i}} e^{-\frac{1}{2}\left(\frac{\ln x-\mu_{i}}{\sigma_{i}}\right)^{2}}, x>0
$$

Its moments are:

$$
E Y_{i}^{n}=e^{n \mu+\frac{1}{2} n^{2} \sigma_{i i}^{2}}, n \in \Re
$$

The $c d f$ is: 


$$
F_{i}\left(x ; \mu_{i}, \sigma_{i}\right)=\Phi\left(\frac{\ln x-\mu_{i}}{\sigma_{i}}\right), x>0,
$$

where $\Phi(x)$ is the standard normal $c d f$. Then

$$
X \sim f(x)=\frac{\partial}{\partial x} F(x), x>0
$$

follows the SLN distribution, with no known closed form. When the summands $Y_{i}$ are independent, $f(x)$ is the convolution of all $f_{i}(x)$ :

$$
f(x)=f_{1}(x) \otimes f_{2}(x) \otimes \cdots \otimes f_{N}(x)
$$

Again, a closed-form method for performing even one of these convolutions is not known.

\subsubsection{Properties}

\subsubsection{Analytically Smooth}

By smooth we mean that all derivatives of the SLN $p d f$ exist everywhere. This can be proved. From (2.5):

$$
\left(\frac{\partial}{\partial x}\right)^{n} f(x)=\left[\left(\frac{\partial}{\partial x}\right)^{n} f_{1}(x)\right] \otimes f_{2}(x) \otimes \cdots \otimes f_{N}(x)
$$

Since the $\mathrm{LN}$ pdf $f_{1}(x)$ has all derivatives, so does the SLN $p d f$. We would like the function approximating the SLN to also have this property, if possible.

\subsubsection{Possibly Unimodal}

It is not evident that the SLN $p d f$ is always unimodal. In fact, a convolution of unimodal functions needs not be unimodal [31]. However, various researchers have worked on trying to estimate the 
mode of the SLN pdf [18], [32]; and simulation results [27], [28] seem to confirm that the SLN $p d f$ is indeed unimodal.

\subsubsection{Heavy-Tailed and Sub-Exponential}

Heavy-tailed (h.t.) and sub-exponential (s.e.) distributions are defined in [33]. The LN distribution is said to be h.t., which is formally defined as follows:

The distribution $F_{i}$ is h.t. if and only if:

$$
\lim _{x \rightarrow+\infty} \frac{1-F_{i}(x+y)}{1-F_{i}(x)}=1, \forall y>0 .
$$

Furthermore, a subclass of h.t. $c d f$ 's is the set of s.e. $c d f$ 's. A distribution is said to be s.e., if and only if:

$$
\lim _{x \rightarrow+\infty} \frac{1-F_{i}^{*_{n}}(x)}{1-F_{i}(x)}=n
$$

where $*_{n}$ indicates the $n$-fold auto-convolution. By L'Hopital's rule, we may write this in terms of $p d f$ :

$$
\lim _{x \rightarrow+\infty} \frac{f_{i}^{*_{n}}(x)}{f_{i}(x)}=n
$$

Now a set of s.e. distributions is not closed under convolution [33]. However, we can show that the SLN for independent terms, or equivalently, the convolution of $N \mathrm{LN} p d f$ 's is s.e., and thus h.t.

Proof: 
It is enough to show that the convolution of any two LN pdf's is s.e., since Theorem 3 in [34] says that if all pair-wise convolutions of a set of $p d f$ 's are s.e., then all manifold convolutions on this set are also s.e..

If two LN pdf's are identical, then their convolution is s.e., by Proposition 2.8 in [33].

If two LN $p d f$ 's are different, then let us call them $f_{1}\left(x ; \mu_{1}, \sigma_{1}\right)$ and $f_{2}\left(x ; \mu_{2}, \sigma_{2}\right)$, such that $\sigma_{1}>\sigma_{2} ;$ or, if $\sigma_{1}=\sigma_{2}$, such that $\mu_{1}>\mu_{2}$. Then:

$$
\begin{gathered}
\lim _{x \rightarrow+\infty} \frac{1-F_{2}(x)}{1-F_{1}(x)}=\lim _{x \rightarrow+\infty} \frac{f_{2}(x)}{f_{1}(x)} \\
=\lim _{x \rightarrow+\infty} \frac{\sigma_{1}}{\sigma_{2}} \exp \left[-\frac{1}{2}\left(\frac{1}{\sigma_{2}^{2}}-\frac{1}{\sigma_{1}^{2}}\right) \ln ^{2} x+\left(\frac{\mu_{2}}{\sigma_{2}^{2}}-\frac{\mu_{1}}{\sigma_{1}^{2}}\right) \ln x-\frac{1}{2}\left(\frac{\mu_{2}^{2}}{\sigma_{2}^{2}}-\frac{\mu_{1}^{2}}{\sigma_{1}^{2}}\right)\right] \\
=\left\{\begin{array}{l}
\lim _{x \rightarrow+\infty} x^{K}=0, K=\frac{\mu_{2}-\mu_{1}}{\sigma_{1}^{2}}<0, \quad \sigma_{1}=\sigma_{2} \\
\lim _{x \rightarrow+\infty} \frac{\sigma_{1}}{\sigma_{2}} \exp \left[K \ln ^{2} x\right]=0, K=-\frac{1}{2}\left(\frac{1}{\sigma_{2}^{2}}-\frac{1}{\sigma_{1}^{2}}\right)<0, \quad \sigma_{1}>\sigma_{2}
\end{array}\right\}=0 .
\end{gathered}
$$

From Proposition 2.7 in [33], we find that the convolution $f_{1} \otimes f_{2}$ is s.e..

This proves that the SLN is s.e. for independent summands. In [21], it was proved that the tail behaviour is the same for correlated summands. Thus, in general, the SLN distribution is subexponential, and thus heavy-tailed.

\subsubsection{Location in a Function Space}

The location of the SLN pdf in a function space is considered in [18]. The LN distribution has two parameters, $\sigma>0, \mu$, and as such can be considered to generate a half-plane in a function space, as shown in Figure 2-1. A LN pdf with small $\left(\sigma^{2} \ll 1\right)$ variance is nearly Gaussian, because the two distributions are related by exponential function, which can be seen as locally 
linear when small variances are involved. Also, since the LN distribution has finite moments (2.3), the Central Limit Theorem applies, and the successive convolutions of LN $p d f$ 's, properly normalised, converges to a Gaussian distribution. However, this convergence will be extremely slow for higher $\sigma^{2} \gg 1$, which is our case of interest in cellular communications: $\sigma \geq 6 d B=1.38$ in linear scale. For these higher variances, the moments (2.3) are very large $\left(E Y_{i}^{2} \gg E^{2} Y_{i}\right.$ ), which makes convergence slow. This can also be seen as a consequence of the SLN distribution being heavy-tailed (see Section 2.1.2.3): because the Gaussian distribution is not h.t., while the sum of any number of LN RV's is, the SLN will not converge to a Gaussian distribution for any finite number $N$ of summands. Several SLN methods, [26], [35]-[37] assume that the trajectory of successive convolutions lies near to the LN half-plane, effectively meaning that the SLN is approximately LN. However, this has proven not to be the case in many situations, and more recent work [27]-[30] suggests that new $c d f$ forms need to be developed for approximating the SLN.

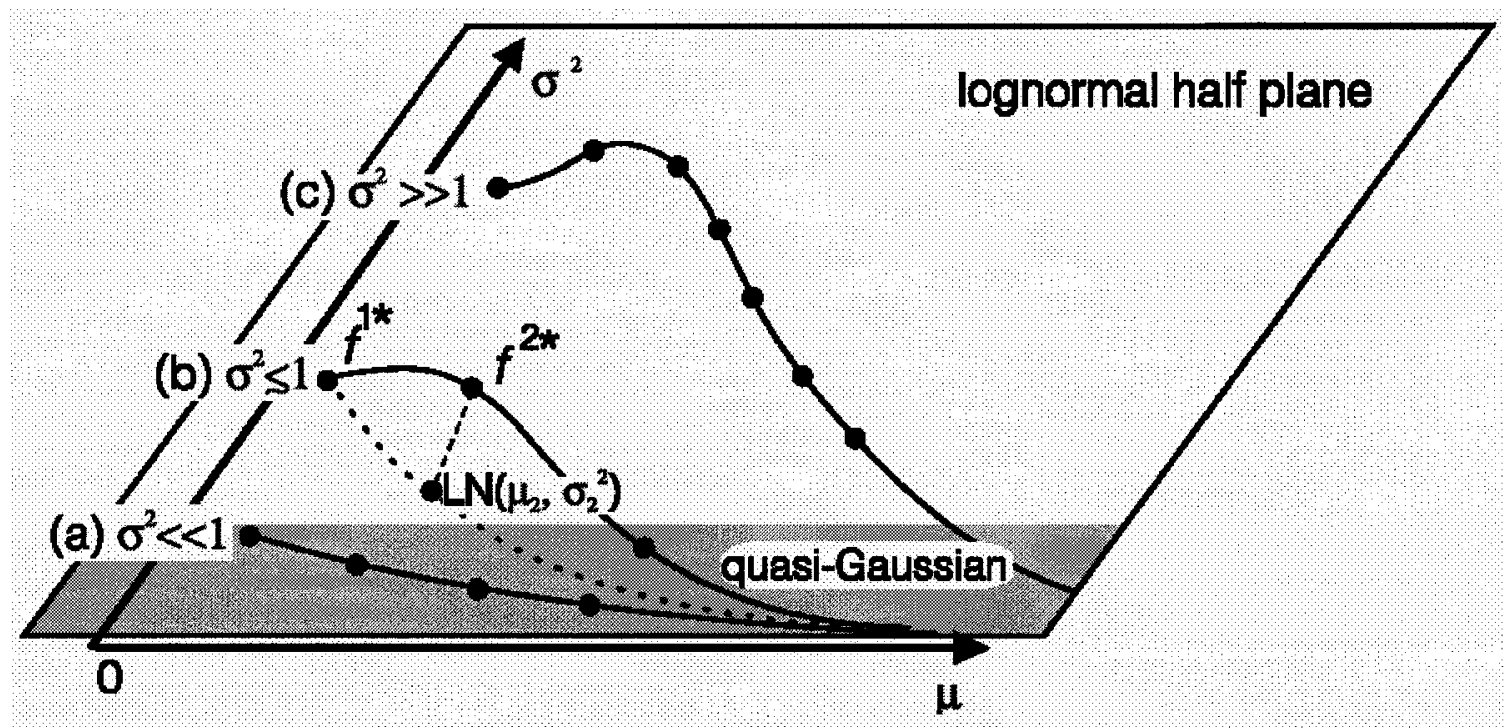

Figure 2-1 Locus of successive convolutions of LN pdfs in a function space. Taken from [18]. 


\subsubsection{Lognormal ProbabIIIty Paper}

\subsubsection{A Bijective Transformation}

It is convenient to look at the $c d f$ of the SLN on lognormal probability paper [35], where LN distributions are mapped onto straight lines by the following transformation $T$ :

$$
T: F(x) \mapsto \tilde{F}(x)=\Phi^{-1}\left(F\left(e^{x}\right)\right)
$$

Indeed this transformation maps:

$$
T: \Phi\left(\frac{\ln x-\mu_{i}}{\sigma_{i}}\right) \mapsto \frac{x-\mu_{i}}{\sigma_{i}}
$$

which is a straight line of strictly positive slope. Conversely, each line of strictly positive slope corresponds to a unique $\mathrm{LN}$ distribution.

\subsubsection{Order Conservation}

\section{Lemma 1:}

For any supports $I, J$, such that $x \in I \Leftrightarrow e^{x} \in J$ we have:

$$
\forall x \in I, \tilde{F}(x)>\frac{x-\mu}{\sigma} \Leftrightarrow \forall x \in J, F(x)>\Phi\left(\frac{\ln x-\mu}{\sigma}\right)
$$

and

$$
\forall x \in I, \tilde{F}(x)<\frac{x-\mu}{\sigma} \Leftrightarrow \forall x \in J, F(x)<\Phi\left(\frac{\ln x-\mu}{\sigma}\right)
$$

Proof: 
Let $g(x)=\tilde{F}(x)-\frac{x-\mu}{\sigma}>0$ and

$T: \Phi\left(\frac{\ln x-\mu}{\sigma}+g(\ln x)\right)=F(x) \mapsto \tilde{F}(x)=\frac{x-\mu}{\sigma}+g(x)$

Since

$\forall x \in I, g(x)>0 \Leftrightarrow \forall x \in J, g(\ln x)>0$ and $\Phi(x)$ is strictly increasing, we have:

$\forall x \in J, F(x)>\Phi\left(\frac{\ln x-\mu}{\sigma}\right)$, which proves (2.14). The proof is analogous for (2.15).

\subsubsection{Concavity}

Based on the all the simulation and numerical curves in literature, it would seem that the SLN $c d f$ is always concave down on $\mathrm{LN}$ paper. This assumption is made in [35]. However, we are not aware of any proof of this.

Because it is concave, the transformed $c d f$ is no longer linear, and in fact becomes less and less so as the number of summands $N$ increases, as seen in Figure 2-2 for independent, identically distributed (i.i.d.) RV's. This means that a LN distribution cannot, in general, be a good approximation to the SLN. 


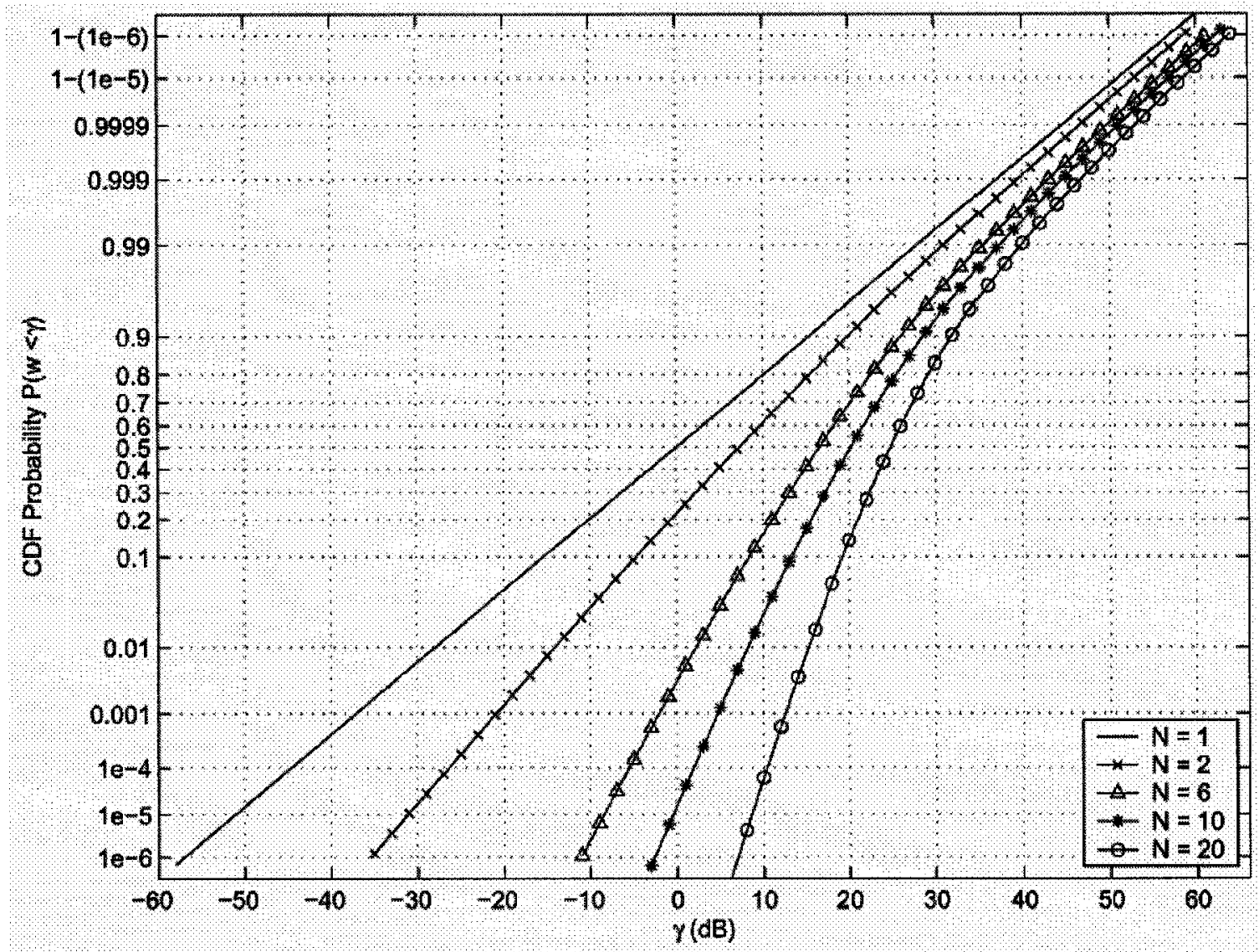

Figure 2-2 cdf on LN paper of the i.i.d. SLN with $\sigma=12 \mathrm{~dB}$. Taken from [35].

\subsubsection{Asymptotes}

Again, looking at all the simulation and numerical curves in literature, it would appear that, on LN paper, the $c d f$ has linear asymptotes at both limits. This would mean that the SLN distribution could be well-approximated by two (different) LN distributions at each tail. This fact is theoretically asserted for the upper tail [21], but there has been no mathematical evidence for there being an asymptote in the lower tail. Also, until now, most SLN methods have completely ignored the nature of the tails of the $c d f$. We explore these asymptotes formally in Chapter 3. 


\subsection{Closed-Form Methods}

We are interested primarily in developing a closed-form solution to our interference problem, which means we are particularly interested in closed-form results on the SLN.

\subsubsection{Positive Moment-Matching}

The simplest approach to approximating the SLN is to assume that the SLN distribution is LN, and match its first two positive moments. This is the first method for solving the SLN problem, and is known as the Fenton-Wilkinson (FW) method [26]. The moments of a LN distribution are known (2.3). Let us use the problem statement in Section 2.1.1, and let us approximate the sum of the SLN RV $X$ by a LN:

$$
X \sim F(x) \approx \Phi\left(\frac{\ln x-\mu_{F W}}{\sigma_{F W}}\right)
$$

The first two moments of the SLN can be calculated as follows [38]:

$$
\begin{gathered}
E[X]=E\left[\sum_{i=1}^{N} Y_{i}\right]=\sum_{i=1}^{N} E\left[Y_{i}\right]=\sum_{i=1}^{N} e^{\mu_{i}+\frac{1}{2} \sigma_{i}^{2}} \\
E\left[X^{2}\right]=E\left[\left(\sum_{i=1}^{N} Y_{i}\right)^{2}\right]=E\left[\sum_{i=1}^{N} Y_{i}^{2}+2 \sum_{i=1}^{N} \sum_{j>i}^{N} Y_{i} Y_{j}\right]=\sum_{i=1}^{N} E\left[Y_{i}^{2}\right]+2 \sum_{i=1}^{N} \sum_{j>i}^{N} E\left[Y_{i} Y_{j}\right] \\
=\sum_{i=1}^{N} e^{2 \mu_{i}+2 \sigma_{i}^{2}}+2 \sum_{i=1}^{N} \sum_{j>i}^{N} e^{\mu_{i}+\mu_{j}+\frac{1}{2}\left(\sigma_{i}^{2}+\sigma_{j}^{2}\right)+p_{i j} \sigma_{i} \sigma_{j}} .
\end{gathered}
$$

Here $\rho_{i j}$ is the correlation coefficient between the Gaussian RV's $\ln Y_{i}$ and $\ln Y_{j}$. These moments are then matched with those of (2.16): 


$$
\left\{\begin{array} { l } 
{ E [ X ] = e ^ { \mu _ { F W } + \frac { 1 } { 2 } \sigma _ { F W } ^ { 2 } } } \\
{ E [ X ^ { 2 } ] = e ^ { 2 \mu _ { F W } + 2 \sigma _ { F W } ^ { 2 } } }
\end{array} \Leftrightarrow \left\{\begin{array}{l}
\mu_{F W}=2 \ln E[X]-\frac{1}{2} \ln E\left[X^{2}\right] \\
\sigma_{F W}^{2}=-2 \ln E[X]+\ln E\left[X^{2}\right]
\end{array}\right.\right.
$$

Matching higher moments has also been proposed [9], [26], [39]. In general, we find the moments of order $n$ and $n+1$ in a manner similar to (2.17), (2.18), then match the moments according to:

$$
\left\{\begin{array} { l } 
{ E [ X ^ { n } ] = e ^ { n \mu _ { F W } + \frac { 1 } { 2 } n ^ { 2 } \sigma _ { F W } ^ { 2 } } } \\
{ E [ X ^ { n + 1 } ] = e ^ { ( n + 1 ) \mu _ { F W } + 2 ( n + 1 ) ^ { 2 } \sigma _ { F W } ^ { 2 } } }
\end{array} \Leftrightarrow \left\{\begin{array}{l}
\mu_{F W}=\frac{n+1}{n} \ln E\left[X^{n}\right]-\frac{n}{n+1} \ln E\left[X^{n+1}\right] \\
\sigma_{F W}^{2}=-\frac{2}{n} \ln E\left[X^{n}\right]+\frac{2}{n+1} \ln E\left[X^{n+1}\right]
\end{array} .\right.\right.
$$

Now matching higher and higher moments of positive RV's tends to give much more weight to the upper tail of the distribution. More specifically:

1. Moment-matching tends to approximate the upper tail much better than the body or the lower tail [26], [35], [40], since moments essentially determine the tail of a distribution $[43]$.

2. By matching higher moments as in (2.20), the overall curve match to the body gets worse and worse, and approximates the far upper tail more and more.

3. Based on the last point, it is possible to perform successive moment-matching for various $n$ and construct a piecewise-LN method [39], [40]. However, we dislike a piecewise solution because it is cumbersome to use.

4. As the moment order $n$ goes to infinity, the summand with the highest moments dominates, and the moment-matching method converges to a LN asymptote determined by the dominating term. This will be explored in more detail in Section 2.2.2. 
In conclusion, moment-matching is a simple-closed form solution to the SLN problem. There exist known expressions for bounds on the error of this method [41].

\subsubsection{Asymptotics}

The behaviour of the SLN distribution $F(x)$ as $x \rightarrow+\infty$ has been previously studied [21], [42]. It was shown in [42] that the moments $E\left[X^{n}\right]$ of a SLN are dominated by the heaviest summand(s) as $n$ increases. In [40], it is suggested that matching higher and higher moments of the sum to those of a LN distribution gives a better and better LN asymptote to the upper tail behaviour. In [43], it is argued that moments of positive RV's (as is our case) determine the upper tail. All this information suggests that looking at the heaviest summand(s) alone will give us a good approximation of the tail.

Reference [21] gives a simple procedure to find the upper tail asymptote:

Choose the summands with the highest logarithmic standard deviation:

$$
\sigma_{U}=\max _{i=1, \ldots, N} \sigma_{i}
$$

Among these summands, chose those with the highest logarithmic mean:

$$
\mu_{U}=\max _{i: \sigma_{i}=\sigma_{U}} \mu_{i}
$$

Count the number of these "heaviest" summands:

$$
\kappa_{U}=\#\left\{\left(\mu_{i}, \sigma_{i}\right)=\left(\mu_{U}, \sigma_{U}\right)\right\} \text {, }
$$

where $\#\{S\}$ is the cardinality of set $S$.

The tail distribution is then that of the heaviest summand(s), multiplied by the number of these heaviest summands: 


$$
\begin{aligned}
& f(x) \stackrel{x \rightarrow+\infty}{\sim} f_{U}(x)=\frac{\kappa_{U}}{\sqrt{2 \pi} x \sigma_{U}} e^{-\frac{1}{2}\left(\frac{\ln x-\mu_{U}}{\sigma_{U}}\right)^{2}} \\
& F(x)^{\stackrel{x \rightarrow+\infty}{\sim}} F_{U}(x)=\kappa_{U} \Phi\left(\frac{\ln x-\mu_{U}}{\sigma_{U}}\right)+\left(1-\kappa_{U}\right) .
\end{aligned}
$$

Note that [21] proves that this result is also valid when the summands with the same marginal distributions are correlated.

\subsubsection{Bounds}

\subsubsection{Tighter, Non-Parametric Approximation}

It is argued in [21] that if a summand is not heaviest, but nearly so, it also contributes significantly to the tail for large but bounded $x$. In [44] are given bounds that are tight in the upper tail. Thus, a better asymptote is the distribution of the maximum of the summands:

$$
\begin{gathered}
\sum_{i=1}^{N} Y_{i} \geq \max _{i} Y_{i} \\
P\left(\sum_{i=1}^{N} Y_{i}<x\right)=F(x) \stackrel{x \rightarrow+\infty}{\sim} F_{U B}(x)=\prod_{i=1}^{N} \Phi\left(\frac{\ln x-\mu_{i}}{\sigma_{i}}\right)=P\left(\max _{i} Y_{i}<x\right) \geq F(x) .
\end{gathered}
$$

Indeed, when we take the sum of positive terms that have high variance, in most outcomes there will be one term that is much larger than the others, and the maximum of the RV's is a good lower bound to their sum. In the i.i.d. case this bound becomes a PLN distribution, which is called Farley's method in literature [45]:

$$
F(x) \stackrel{x \rightarrow+\infty}{\sim} \Phi^{N}\left(\frac{\ln x-\mu}{\sigma}\right),\left[\mu_{i}, \sigma_{i}\right]=[\mu, \sigma] \forall i .
$$

We also have: 


$$
\begin{gathered}
\sum_{i=1}^{N} Y_{i} \leq N \max _{i} \\
P\left(\sum_{i=1}^{N} Y_{i}<x\right)=F(x) \stackrel{x \rightarrow+\infty}{\sim} F_{L B}(x)=\prod_{i=1}^{N} \Phi\left(\frac{\ln x-\ln N-\mu_{i}}{\sigma_{i}}\right)=P\left(N \max _{i} Y_{i}<x\right) \leq F(x) .
\end{gathered}
$$

Equations (2.26) and (2.28) are upper and lower bounds respectively to the SLN $c d f$, which are called lower and upper bounds respectively in [44], since they are used to refer either to the complementary $c d f$ or to the value of the RV itself.

\subsubsection{Arithmetic-Geometric Mean Inequality}

While the sum of LN RV's is difficult to find, the product of LN is very simple. Let

$$
Z=\prod_{i=1}^{N} Y_{i}
$$

Then $Z$ is simply a LN RV with parameters:

$$
\mu_{z}=\sum_{i=1}^{N} \mu_{i}, \quad \sigma_{z}^{2}=\sum_{i=1}^{N} \sigma_{i}^{2}
$$

assuming Yi are independent.

The method proposed in [46] uses the geometric-arithmetic (AG) mean inequality: for any positive quantities, their geometric mean is always upper-bounded by their arithmetic mean:

$$
\forall V \mid V=\left\{x_{i} \geq 0\right\}_{i=1}^{N}: \frac{1}{N} \sum_{i=1}^{N} x_{i} \geq\left(\prod_{i=1}^{N} x_{i}\right)^{\frac{1}{N}}
$$

In our SLN case, 


$$
X=\sum_{i=1}^{N} Y_{i} \geq N\left(\prod_{i=1}^{N} Y_{i}\right)^{\frac{1}{N}}=N Z^{\frac{1}{N}}
$$

from which we obtain a bound on the SLN distribution:

$$
F(x)=P(X<x) \leq P\left(N Z^{\frac{1}{N}}<x\right)=P\left(Z<\left(\frac{x}{N}\right)^{N}\right)=\Phi\left(\frac{N \ln x-\mu_{Z}-N \ln N}{\sigma_{Z}}\right) .
$$

Now let us examine the inequality (2.31) with some numerical examples. Take $V=\{4,5,6\}$, where all the terms are of the same order of magnitude. Then the arithmetic mean is 5 , and the geometric mean is $4.932 \ldots$ Not only is the bound correct, it is also very tight in this case. Let us take another example: $V=\{1,10,100\}$, with different orders of magnitude. Then the arithmetic mean is 37 , and the geometric mean is 10 , which is a very loose lower bound.

From this we come to the conclusion that if our LN summands are of different $\mu_{i}$ or of high $\sigma_{i}$, they will take values of very different orders of magnitude. This is indeed the case in our problem, where there are interferers located both near and far, and the LN shadowing is of at least $6 \mathrm{~dB}$. Hence the bound in (2.33) will be very loose, and essentially useless.

This bound is however very useful in solving another problem: calculating the total interference power from in-cell users in a power-controlled Code Division Multiple Access (CDMA) system. In this case, all receive powers are essentially the same, but vary slightly in a LN manner, say by $1 \mathrm{~dB}$, due to imperfect PC. In this case the method, which was only tested for up to $4 \mathrm{~dB}$, is good for $4 \mathrm{~dB}$ and excellent for $1 \mathrm{~dB}[46]$. 


\subsection{Numerical Methods}

The methods in this class consist in calculating numerical integrals, perhaps iteratively, and perhaps in several dimensions.

\subsubsection{Parametric Methods}

Here are methods that use numerical integration in order to find the parameters of some function that approximates the SLN $c d f$.

\subsubsection{Logarithmic Moment-Matching: Schwartz - Yeh}

This is one of the earliest and most important methods, originally published in [37], it was used in [1], [47] to analyse outage probability in cellular systems. The method is described in [38], so we only give a brief description here:

The SY method approximates the SLN by a LN distribution. Moment-matching is performed in the logarithmic, instead of the linear, domain. The sum of the first two LN RV's is approximated by a partial sum $X_{2}$, which is LN with parameters $\mu_{X_{2}}, \sigma_{X_{2}}$ which are found by solving:

$$
\begin{gathered}
E\left[\ln X_{2}\right]=\mu_{X_{2}}=E\left[\ln \left(Y_{1}+Y_{2}\right)\right] \\
E\left[\ln X_{2}^{2}\right]=\mu_{X_{2}}^{2}+\sigma_{X_{2}}^{2}=E\left[\ln ^{2}\left(Y_{1}+Y_{2}\right)\right]
\end{gathered}
$$

Equation (2.34) is reformulated as follows: 


$$
\begin{gathered}
\mu_{X_{2}}=E\left[\ln Y_{1}\right]+E\left[\ln \left(1+\frac{Y_{2}}{Y_{1}}\right)\right] \\
=\mu_{1}+\int^{+\infty} \ln (1+x) \frac{1}{\sqrt{2 \pi} x\left(\sigma_{1}^{2}+\sigma_{2}^{2}\right)} \exp \left[-\frac{1}{2}\left(\frac{\ln x-\left(\mu_{2}-\mu_{1}\right)}{\sqrt{\sigma_{1}^{2}+\sigma_{2}^{2}}}\right)^{2}\right] d x
\end{gathered}
$$

This formulation is due to the fact that $Y_{2} / Y_{1}$ is also $\mathrm{LN}$. The integral cannot be calculated in closed form and is thus left to numerical integration. A similar formulation is used to find $\sigma_{X_{2}}$.

Once the sum of two LN RV's has been approximated by another LN, we proceed iteratively by solving in the same manner:

$$
\begin{gathered}
E\left[\ln X_{n}\right]=\mu_{X_{n}}=E\left[\ln \left(X_{n-1}+Y_{n}\right)\right], \\
E\left[\ln X_{n}^{2}\right]=\mu_{X_{n}}^{2}+\sigma_{X_{n}}^{2}=E\left[\ln ^{2}\left(X_{n-1}+Y_{n}\right)\right] .
\end{gathered}
$$

This is a recursive method with respect to the number of summands. In total we perform $N-1$ iterations, each involving a numerical integral. In order to calculate this integral more efficiently and accurately, modified methods have been proposed [48], [49].

\subsubsection{Log-Shifted Gamma Function: Le-Ngoc - Lam}

The LL method was proposed in [27], and is inspired by the SY method. A three-parameter function: a log-shifted Gamma (LSG) function (see Section 3.2.1.1), is used as an approximating $c d f$. The sum of two LN RV's is approximated by a LSG, after which each successive partial sum is computed as the sum of a LN and a LSG RV. The three parameters of each successive LSG distribution are found through matching the first two logarithmic moments of the sum (as in SY), and also the first linear moment (as in FW). This method also requires $N-1$ iterations, and numerical integrations at every step. The method is expanded to include the sum of correlated LN RV's in [50]. 


\subsubsection{Pearson Type IV Function: Zhang - Song}

A four-parameter Pearson Type IV (see Section 3.2.1.4) $c d f$ is used by Zhang - Song (ZS) in [28] as an approximating distribution to the logarithm of a SLN. These parameters are found by matching for the first four moments of the logarithm of a sum of LN RV's, given by:

$$
E\left[\ln X^{n}\right]=E\left[\ln ^{n}\left(\sum_{i=1}^{N} Y_{i}\right)\right], n=1,2,3,4
$$

It is not specified how these moments are calculated, but it is probably through numerical integration.

\subsubsection{Negative Moment-Matching}

Rather than matching positive moments of the SLN as in Section 2.2.1, [21] proposed to match the first two negative moments (powers -2 and -1 ). The advantage of this method is that it gives less weight to the higher tails, and more to the lower portion of the $c d f$. The disadvantage is that there is no closed-form expression for the negative moments of a sum of RV's, and an $\mathrm{N}$ dimensional numerical integration is necessary.

This method was extended in [40] so as to use (2.20) for both positive and negative $n$ except 0 and -1 . This gives us a piecewise solution that is accurate in both tails, but again requires numerical integration for negative $n$, which give the lower tail. The method does not yield a very exact match in the body (centre) of the $c d f$ though, and produces a piecewise solution.

\subsubsection{Flexible Lognormal Approximation: Wu - Mehta - Zhang}

The WMZ method presented in [36] approximates the SLN $c d f$ by a LN. However, the LN $c d f$ can be matched to any part of the SLN curve, by selecting parameters correctly. The method uses the LN distribution's moment-generating function. Since its closed form is not known, a short 
Gauss-Hermite expansion of less than 20 terms is used. This method has the advantages of providing a piecewise $\mathrm{LN}$ solution of arbitrarily good accuracy given enough pieces, and this without recursion. However a non-linear equation still needs to be solved numerically.

The method is expanded in [51] to include correlated LN and LN-Rice RV's.

\subsubsection{Characteristic Function Evaluation}

Another numerical approach to the SLN problem for independent summands is to take (2.6) and consider it in the Fourier domain: the characteristic function ( $c f$ ) of a sum of independent RV's is the product of their individual $c f$ s. Now the $c f$ of a LN RV is not known in closed form, but can be found numerically. This results in an integral with a very long oscillating tail, and the integral converges very slowly. The first numerical study of the LN $c f$ seems to be [19]. The modified Clenshaw-Curtis method is used in [35], while [52] uses a new method where the integral is transformed so that in no longer oscillates. Reference [53] proposes yet another integral. The $c f$ method is also treated mathematically in [22], where it is shown that the SLN $c d f$ for independent terms can be expressed as a sum of products of LN terms.

\subsection{Curve-Fitting Methods}

The following methods could be called "empirical", because they consist in already knowing the SLN $c d f$ as a vector of values, and then fitting the best possible analytical curve to it. The SLN $c d f$ is obtained numerically by methods from Section 2.3 .2 . These methods essentially correspond to a tabulation of curve parameters against the input parameters of the summed LN RV's. The weakness of this approach is that there exists an almost infinite variety of cases for the set of input parameters $\left\{\mu_{i}, \sigma_{i}\right\}_{i=1}^{N}$. Thus these methods only examine i.i.d. cases, where there are only two parameters: $N$ and $\sigma$, while $\mu$ can be set to zero without loss of generality. 


\subsubsection{Minimax Lognormal Fitting: Beaulieu - Xie}

After using the modified Clenshaw-Curtis method to find the $c f$, the BX method [35] assumes that the SLN $c d f$ is concave on LN paper (see Section 2.1.4.3), and fits the best possible straight line on the LN paper to the SLN $c d f$ using the minimax criterion over the range $\left[10^{-6}, 1-10^{-6}\right]$ of probabilities. The result is a LN approximation to the SLN distribution that does not privilege any particular region of the curve.

\subsubsection{Three-Parameter Function: Beaulieu - Rajwani}

The previous method (or any other method approximating the SLN by a LN) cannot be accurate everywhere, because in most cases the SLN $c d f$ is not approximately LN (see Section 2.1.4.3). Another $c d f$ is proposed by Beaulieu - Rajwani [30] (BR), which will be analysed in detail in Section 3.2.1.2. Here we will only say that the distribution has three parameters, which are found by a non-linear least-squares fit to the $c d f$ obtained numerically. These parameters are tabulated for $N=2$ to 20 , and $\sigma=6 \mathrm{~dB}$ and $12 \mathrm{~dB}$.

\subsubsection{Three-Parameter Function: Zhao - Ding}

Another approach, similar to the previous one, uses also a three-parameter $c d f$ that is parabolic under the transformation $T$. This is the ZD method [29], whose $c d f$ will be examined in detail in Section 3.2.1.3. Beaulieu and Rajwani mention that they have also tried and discarded a parabolic function [30]. Our simulation results in Figure 3-2 show that the BR method greatly outperforms the ZD method. However, the ZD method has the advantage of being defined for a whole range of $N$ and $\sigma$. 


\subsection{Validation of Methods}

Given so many methods to choose from, we would like to analyse and compare them in order to choose the ones most suited to our purpose.

\subsubsection{Comparisons in Literature}

There are several papers in literature dealing with comparing FW and SY methods: [45] argues that FW is better in several cases, while [38] prefers SY. In fact which method is better depends on the choice of LN parameters [48]. Also, FW tends to approximate the upper tail better, while SY approximates the body, or middle portion of the $c d f$, as can be seen in [27], [35], [45]. Also Farley's method is always asymptotic to the SLN $c d f$ in the upper tail, but is not very good in the lower tail, sometimes better and sometimes worse than FW [27], [35], [45].

\subsubsection{Comparison and Classification of Methods}

There exist various approximate solutions to the SLN, which generally consist of two distinct parts: choosing a general form for the SLN distribution, and obtaining its parameters for particular cases. One approach has been to approximate the SLN distribution by a LN, and various methods were used to find the best-fitting parameters: FW [26], BX [35], WMZ [36], SY [37], negative moment-matching [40], [54], and LN bound [46]. However, it has become clear that as the number of summands increases, the SLN distribution appears less and less LN (see Figure 2-2). There also exist other methods, which use more complex distribution functions with three or more free parameters: LL [27], ZS [28], ZD [29], and BR [30]. Nonparametric solutions have also been proposed: piecewise-lognormal [26], [39], [40], or product-of-lognormal (Farley's method [45], other bounds [44]). Each of these methods has its drawbacks: the first class of methods, where a LN approximation is used, cannot possibly be accurate over the entire range of $x$. Many methods require numerical integration to find the required parameters: [27], [28], [37], 
[40], [54]. While the behaviour of the upper tail, i.e., as $x \rightarrow \infty$ is known [21], [42], that of the lower tail, i.e., as $x \rightarrow 0$ has no simple expression. Some methods have only been verified for the case of i.i.d. summands [29], [30]. Also, we will show that methods that match the body of the distribution well [27]-[30], are not necessarily accurate in the far tails. On the other hand, the piecewise-lognormal method proposed in [40] does give a good behaviour at the tails, but again requires numerical integration in $N$ dimensions and does not perform as well in the body. Hence, there is no single method that really gives a complete solution to the problem, and there is no closed form for the lower tail.

Figure 2-3 shows the various methods compared according to both accuracy (precision) of the approximation (which is not entirely an objective measure, as different parts of the curve may be better approximated by different methods, and for different parameter choices.) and also by complexity. Complexity is again a partly subjective measure. We tried to estimate the difficulty of understanding and implementing a specific method, while also considering the level of integration required (iterative, multi-variate?). We also included our two new methods, PLN and 4P, introduced in Chapter 3, for comparison. We have indicated by an arrow that the advantageous region for a SLN method is both high precision and low complexity. As can be seen from the Figure, there are no known methods in that region, which indicates that the SLN problem remains essentially open.

Table 2-1 shows a history of the principal developments in the field of the SLN problem in the electrical engineering community. Some methods only apply to i.i.d. or independent but not necessarily identically distributed (i.n.i.d.) RV's. It does not include work on $c f$ 's or comparative work. 


\begin{tabular}{|c|c|c|c|c|}
\hline Method & Form & Statistics Matched & Complexity & Scope \\
\hline FW ('60) [26] & LN & $E[X], E\left[X^{2}\right]$ & Closed-form & any \\
\hline Schleher ('76) [39] & Piece-LN & $\begin{array}{c}\text { Cumulants: } \\
E\left[(X-E[X])^{n}\right]\end{array}$ & Closed-form & i.i.d. \\
\hline SY ('82) [37], [38] & $\mathrm{LN}$ & $E[\ln X], E\left[\ln ^{2} X\right]$ & $\begin{array}{l}\text { Num. integrals, } \\
N-1 \text { recursion }\end{array}$ & any \\
\hline Farley (?) [45] & PLN & $\max _{i} Y_{i} \leq \sum_{i=1}^{N} Y_{i}$ & Closed-form & any \\
\hline Bounds ('01) [44] & $\begin{array}{l}\text { Product } \\
\text { of } \mathrm{LN}\end{array}$ & $\begin{array}{c}\max _{i} Y_{i} \leq \sum_{i=1}^{N} Y_{i} \\
\leq N \max _{i} Y_{i}\end{array}$ & Closed-form & i.n.i.d. \\
\hline BX ('04) [35] & $\mathrm{LN}$ & Minimax fit & $\begin{array}{l}\text { Numerically } \\
\text { computed } c d f\end{array}$ & any \\
\hline AG ('04) [46] & LN & $\frac{1}{N} \sum_{i=1}^{N} Y_{i} \geq\left(\prod_{i=1}^{N} Y_{i}\right)^{\frac{1}{N}}$ & Closed-form & any \\
\hline BR ('04) [30] & (3p.) & $\begin{array}{c}\text { Non-linear } \\
\text { least-squares fit }\end{array}$ & $\begin{array}{l}\text { Numerically } \\
\text { computed } c d f\end{array}$ & i.i.d. \\
\hline LL ('05) [27], [50] & LSG (3p.) & $\begin{array}{c}E[X], E[\ln X] \\
E\left[\ln ^{2} X\right]\end{array}$ & $\begin{array}{l}\text { Num. integrals, } \\
N-1 \text { recursion }\end{array}$ & any \\
\hline WMZ ('05) [36], [51] & $\mathrm{LN}$ & $\begin{array}{l}\text { Moment-generating } \\
\text { function }\end{array}$ & $\begin{array}{l}\text { Num. equations, } \\
\text { Non-recursive }\end{array}$ & any \\
\hline $\begin{array}{c}\text { Negative Moment } \\
\text { Match. ('05) [40], [54] }\end{array}$ & Piece-LN & $\begin{array}{c}E\left[X^{n}\right], E\left[X^{n+1}\right] \\
n=\ldots-3,-2,1,2,3, \ldots\end{array}$ & $\begin{array}{l}\text { Num. integrals } \\
\text { in } N \text { dimensions }\end{array}$ & i.n.i.d. \\
\hline ZD ('06) [29] & (3p.) & $\begin{array}{c}\text { Quadratic } \\
\text { least-squares fit }\end{array}$ & $\begin{array}{l}\text { Numerically } \\
\text { computed } c d f\end{array}$ & i.i.d. \\
\hline ZS ('06) [28] & $\begin{array}{l}\text { Pearson } \\
\text { IV (4p.) }\end{array}$ & $E\left[\ln ^{n} X\right], n=1, \ldots, 4$ & Closed-form ${ }^{*}$ & any \\
\hline
\end{tabular}

* It is not explained how the moments of the logarithm of the SLN are found. Once these are found, the method is closed-form.

Table 2-1 Summary of properties of SLN methods. 


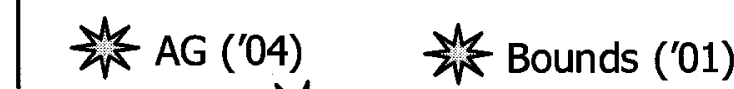
$i^{M} 4 P(' 06)$

Closed Form Positive Moment-Matching: FW ('60)
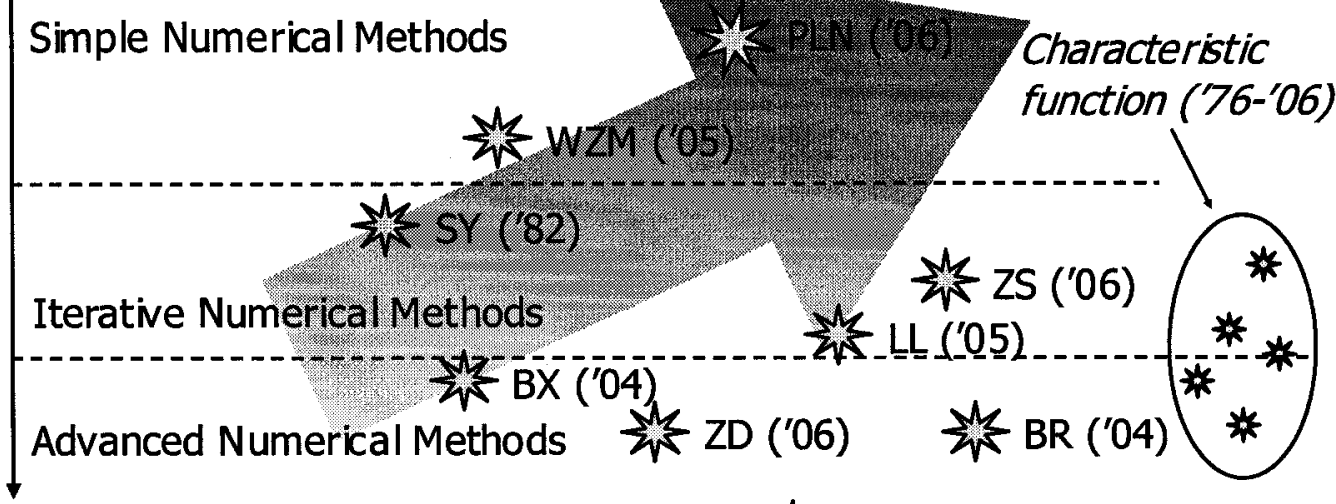

Complexity

许 Negative Moment-Matching ('05)

Figure 2-3 Comparison of SLN methods by complexity and precision.

\subsubsection{Monte-Carlo Simulation Algorithm}

Though various numerical methods exist to find the exact SLN $c d f$ (see Section 2.3.2), we preferred to use Monte-Carlo simulations due to its simplicity in concept and implementation. The algorithm flowchart is given in Figure 2-4. The strongest drawback of this method is the long computational time. We mitigate this problem by letting the simulations run overnight.

We use the simulated curve as a benchmark for evaluating existing SLN methods, or, in Chapter 3, methods of our own device. We will also give an algorithm that efficiently simulates the far lower tail of the SLN $c d f$ in Section 3.1.3.5. 


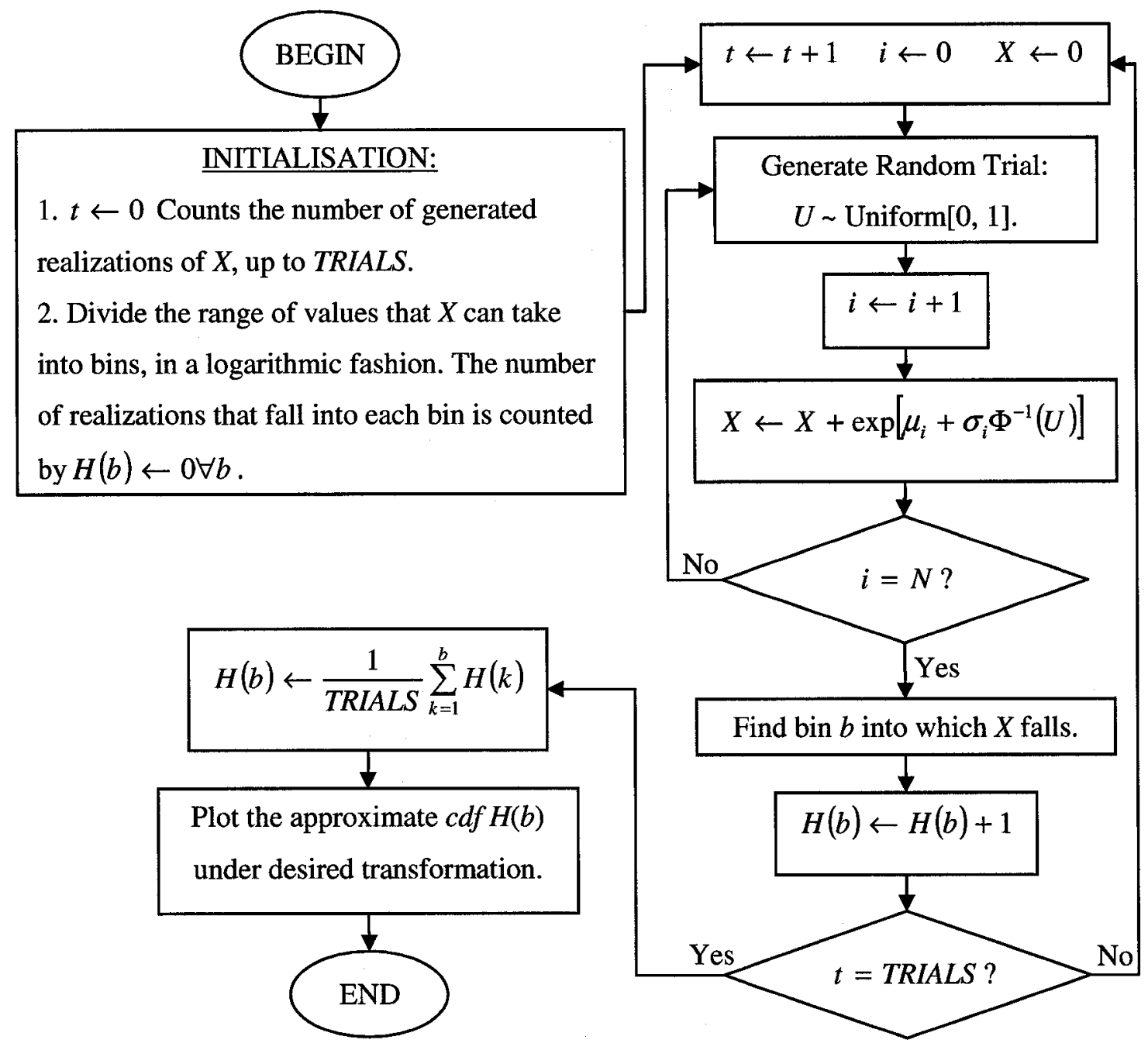

Figure 2-4 Algorithm that simulates the SLN cdf. 


\section{Chapter 3}

\section{Towards a Good, Simple, Closed-Form Approximation of the Distribution of the Sum of Lognormal Random Variables}

In Chapter 2, we have made a thorough search of existing SLN methods and found that there is still no closed-form method that approximates the SLN $c d f$ well. In particular, the closed-form methods gave us some good results in the upper tail, but failed in the lower tail. In this chapter, we develop a closed-form approximation to the lower tail, and combine it with other closed-form results to produce new, more exact closed-form or nearly-closed-form methods.

While it can be argued that knowledge of the tails of the distribution is less important than that of the body, we will show that theoretical results on the tails provide information on the body shape also, because the distribution is smooth (see section 2.1.2.1). Also, in order to apply moment matching on positive RV's, it is important to understand the upper tail [43], since a small error in the distribution at high values may translate into a large error in the moment.

\subsection{Best Lognormal Fit to Either Tail}

In Section 2.1.4.4 we have seen that, on lognormal paper, the tails of the SLN distribution seem to have linear asymptotes, which would indicate that the distribution is approximately LN in either tail. We will now examine this formally by defining a property of tails of distributions, the best lognormal fit (blf), and showing what it means in terms of convergence. We then examine the properties of both tail of the SLN distribution using this framework.

\subsubsection{Definition}

Let $T: F(x) \mapsto \tilde{F}(x)$. Then $\frac{x-\mu}{\sigma}$ is an asymptote to $\tilde{F}(x)$ at $l= \pm \infty$ if and only if: 


$$
\lim _{x \rightarrow l} \tilde{F}(x)-\frac{x-\mu}{\sigma}=0
$$

We say that $F(x)$ has a best lognormal fit $\Phi\left(\frac{\ln x-\mu}{\sigma}\right)$ at $e^{l}$. At any given tail, a cdf may have at most one blf.

\subsubsection{Best Lognormal Fit in the Linear Domain}

We show that if, on lognormal paper, a line $L$ is not the blf to a curve $C$ at the limit $l$, then the corresponding distributions have tails in the corresponding limit $e^{l}$ that are not comparable at any order of magnitude, i.e., their ratio will diverge. In other words, a necessary (but not sufficient) condition for approximating a distribution by a $\mathrm{LN}$ at a given tail is that the two distributions be asymptotes to each other on lognormal paper.

\section{Lemma 2:}

If $\lim _{x \rightarrow-\infty} \tilde{F}(x)-\frac{x-\mu}{\sigma} \neq 0$ or does not exist, then $\lim _{x \rightarrow 0} \frac{F(x)}{\Phi\left(\frac{\ln x-\mu}{\sigma}\right)}=0,+\infty$ or does not exist.

Likewise, if $\lim _{x \rightarrow+\infty} \tilde{F}(x)-\frac{x-\mu}{\sigma} \neq 0$ or does not exist, then $\lim _{x \rightarrow+\infty} \frac{1-F(x)}{1-\Phi\left(\frac{\ln x-\mu}{\sigma}\right)}=0,+\infty$ or does not exist.

Proof:

$$
\lim _{x \rightarrow-\infty} \tilde{F}(x)-\frac{x-\mu}{\sigma} \neq 0 \text { or does not exist }
$$




$$
\begin{gathered}
\Rightarrow \neg\left(\lim _{x \rightarrow-\infty} \tilde{F}(x)-\frac{x-\mu}{\sigma}=0\right) \Leftrightarrow \neg\left(\forall \varepsilon>0, \exists \delta, \forall x<\delta,\left|\tilde{F}(x)-\frac{x-\mu}{\sigma}\right|<\varepsilon\right) \\
\Leftrightarrow \exists \varepsilon>0, \forall \delta, \exists x<\delta,\left|\tilde{F}(x)-\frac{x-\mu}{\sigma}\right| \geq \varepsilon \\
\Leftrightarrow \exists \varepsilon>0, \forall \delta, \exists x<\delta, \tilde{F}(x) \geq \frac{x-(\mu-\sigma \varepsilon)}{\sigma} \vee \tilde{F}(x) \leq \frac{x-(\mu+\sigma \varepsilon)}{\sigma} .
\end{gathered}
$$

Applying Lemma 1:

$$
\begin{aligned}
& \exists \varepsilon>0, \forall \delta, \exists x<\delta, \tilde{F}(x) \geq \frac{x-(\mu-\sigma \varepsilon)}{\sigma} \\
& \Leftrightarrow \exists \varepsilon>0, \forall \delta, \exists x: 0<x<e^{\delta}, F(x) \geq \Phi\left(\frac{\ln x-(\mu-\sigma \varepsilon)}{\sigma}\right) \\
& \Leftrightarrow \exists \varepsilon>0, \forall \delta, \exists x: 0<x<e^{\delta}, \frac{F(x)}{\Phi\left(\frac{\ln x-\mu}{\sigma}\right)} \geq \frac{\Phi\left(\frac{\ln x-(\mu-\sigma \varepsilon)}{\sigma}\right)}{\Phi\left(\frac{\ln x-\mu}{\sigma}\right)} \\
& \left.\Leftrightarrow-\forall \varepsilon>0, \exists \delta, \forall x: 0<x<e^{\delta}, \frac{F(x)}{\Phi\left(\frac{\ln x-\mu}{\sigma}\right)}<\frac{\Phi\left(\frac{\ln x-(\mu-\sigma \varepsilon)}{\sigma}\right)}{\Phi\left(\frac{\ln x-\mu}{\sigma}\right)}\right) \\
& \Rightarrow-\left(\forall \varepsilon>0, \lim _{x \rightarrow 0} \frac{F(x)}{\Phi\left(\frac{\ln x-\mu}{\sigma}\right)}<\lim _{x \rightarrow 0} \frac{\Phi\left(\frac{\ln x-(\mu-\sigma \varepsilon)}{\sigma}\right)}{\Phi\left(\frac{\ln x-\mu}{\sigma}\right)}=\lim _{x \rightarrow 0} \frac{\partial \Phi\left(\frac{\ln x-(\mu-\sigma \varepsilon)}{\sigma}\right)}{\partial \Phi\left(\frac{\ln x-\mu}{\sigma}\right)}\right) \\
& \Leftrightarrow \forall \varepsilon>0, \lim _{x \rightarrow 0} \frac{F(x)}{\Phi\left(\frac{\ln x-\mu}{\sigma}\right)} \geq \lim _{x \rightarrow 0} \exp \left(-\frac{1}{2} \varepsilon^{2}-\varepsilon \frac{(\ln x-\mu)}{\sigma}\right)=+\infty \Rightarrow \lim _{x \rightarrow 0} \frac{F(x)}{\Phi\left(\frac{\ln x-\mu}{\sigma}\right)}=+\infty \text {. }
\end{aligned}
$$

Likewise: 


$$
\begin{gathered}
\exists \varepsilon>0, \forall \delta, \exists x<\delta, \tilde{F}(x) \leq \frac{x-(\mu+\sigma \varepsilon)}{\sigma} \\
\Leftrightarrow \exists \varepsilon>0, \forall \delta, \exists x<e^{\delta}, F(x) \leq \Phi\left(\frac{\ln x-(\mu+\sigma \varepsilon)}{\sigma}\right) \\
\Rightarrow \lim _{x \rightarrow 0} \frac{F(x)}{\Phi\left(\frac{\ln x-\mu}{\sigma}\right)} \leq \lim _{x \rightarrow 0} \frac{\Phi\left(\frac{\ln x-(\mu+\sigma \varepsilon)}{\sigma}\right)}{\Phi\left(\frac{\ln x-\mu}{\sigma}\right)}=\lim _{x \rightarrow 0} \exp \left(-\frac{1}{2} \varepsilon^{2}+\varepsilon \frac{(\ln x-\mu)}{\sigma}\right)=0 .
\end{gathered}
$$

But $\forall x, \frac{F(x)}{\Phi\left(\frac{\ln x-\mu}{\sigma}\right)} \geq 0$, thus:

$$
\exists \varepsilon>0, \forall \delta, \exists x<\delta, \tilde{F}(x)<\frac{x-(\mu+\sigma \varepsilon)}{\sigma} \Rightarrow \lim _{x \rightarrow 0} \frac{F(x)}{\Phi\left(\frac{\ln x-\mu}{\sigma}\right)}=0 \text { or does not exist. }
$$

Thus, if $\lim _{x \rightarrow-\infty} \tilde{F}(x)-\frac{x-\mu}{\sigma} \neq 0$ or does not exist, then $\lim _{x \rightarrow 0} \frac{F(x)}{\Phi\left(\frac{\ln x-\mu}{\sigma}\right)}$ is $0,+\infty$, or does not exist. The proof for $x \rightarrow+\infty$ is analogous. This proves Lemma 2.

Lemma 2 has several important implications:

1. If a distribution has no linear asymptote in the $\mathrm{LN}$ domain at limit $l$, then it cannot possibly be approximated by a $\mathrm{LN}$ function in the linear domain at the corresponding tail.

2. If the ratio of two $p d f$ 's converges to a non-zero constant at limit $l$, and one of the two distributions has a blf, then the other must have the same blf.

\subsubsection{Lower Tail (for Independent Summands)}

To our knowledge, there is no work dealing directly with the lower tail behaviour of the SLN. There is some literature on the lower tail of the distribution of the sum of positive RV's, but it 
does not readily apply to the SLN. The polynomial conditions required in [55] are not fulfilled by the LN distribution.

\subsubsection{Discrete Convolution Approximation}

In order to find the lower tail of the distribution of the sum of two independent LN RV's with $p d f^{\prime}$ s $f_{1}(x)$ and $f_{2}(x)$, we need to find the convolution near 0 of two LN $p d f$ 's. We proceed as follows: let us sample the $c d f$ 's at $0, x, 2 x, \ldots$, where $x$ is small. Then, we perform a discrete convolution and look at the first non-zero sample:

$$
f(2 x) \stackrel{x \rightarrow 0}{\approx} x f_{1}(x) f_{2}(x)
$$

Proceeding likewise for $N$ functions:

$$
\begin{gathered}
f(N x) \stackrel{x \rightarrow 0}{\approx} x^{N-1} \prod_{i=1}^{N} f_{i}(x) . \\
f(N x) \stackrel{x \rightarrow 0}{\approx} \frac{1}{x} \sqrt{2 \pi}^{-N} \prod_{i=1}^{N} \sigma_{i}^{-1} e^{-\frac{1}{2} \sum_{i=1}^{N} \frac{\left(\ln x-\mu_{i}\right)^{2}}{\sigma_{i}^{2}}}=\frac{1}{x} \sqrt{2 \pi}^{-N} \prod_{i=1}^{N} \sigma_{i}^{-1} e^{-\frac{1}{2} \ln ^{2} x \sum_{i=1}^{N} \frac{1}{\sigma_{i}^{2}}+\ln x \sum_{i=1}^{N} \frac{\mu_{i}}{\sigma_{i}^{2}}-\frac{1}{2} \sum_{i=1}^{N} \frac{\mu_{i}^{2}}{\sigma_{i}^{2}} .}
\end{gathered}
$$

Let

$$
\sigma_{L}=\left(\sum_{i=1}^{N} \frac{1}{\sigma_{i}^{2}}\right)^{-\frac{1}{2}}
$$

then: 


$$
\begin{aligned}
& f(N x) \stackrel{x \rightarrow 0}{\approx}=\frac{1}{x} \sqrt{2 \pi}^{-N} \prod_{i=1}^{N} \sigma_{i}^{-1} e^{-\frac{1}{2} \frac{1}{\sigma_{L}^{2}}\left(\ln ^{2} x-2 \ln x \sigma_{L}^{2} \sum_{i=1}^{N} \frac{\mu_{i}}{\sigma_{i}^{2}}+\sigma_{L}^{2} \sum_{i=1}^{N} \frac{\mu_{i}^{2}}{\sigma_{i}^{2}}\right)} \\
& =\frac{1}{x} \sqrt{2 \pi}^{-N} \prod_{i=1}^{N} \sigma_{i}^{-1} e^{-\frac{1}{2} \frac{1}{\sigma_{L}^{2}}\left(\left(\ln x-\sigma_{L}^{2} \sum_{i=1}^{N} \frac{\mu_{i}}{\sigma_{i}^{2}}\right)^{2}-\left(\sigma_{L}^{2} \sum_{i=1}^{N} \frac{\mu_{i}}{\sigma_{i}^{2}}\right)^{2}+\sigma_{L}^{2} \sum_{i=1}^{N} \frac{\mu_{i}^{2}}{\sigma_{i}^{2}}\right)} \\
& =\frac{1}{x} \sqrt{2 \pi}^{-N} \prod_{i=1}^{N} \sigma_{i}^{-1} e^{-\frac{1}{2}\left(\frac{\ln x-\sigma_{L}^{2} \sum_{i=1}^{N} \frac{\mu_{i}}{\sigma_{i}^{2}}}{\sigma_{L}}\right)^{2}+\frac{1}{2} \sigma_{L}^{2}\left(\sum_{i=1}^{N} \frac{\mu_{i}}{\sigma_{i}^{2}}\right)^{2}-\frac{1}{2} \sum_{i=1}^{N} \frac{\mu_{i}^{2}}{\sigma_{i}^{2}}} .
\end{aligned}
$$

\subsubsection{Scaled Lognormal Approximation}

From the above approximation, it would seem that we can express the lower tail of the SLN distribution as a $\mathrm{LN}$ times constant distribution:

$$
f(x) \stackrel{x \rightarrow 0}{\approx} \frac{\kappa_{L}}{\sqrt{2 \pi} \sigma_{L} x} e^{-\frac{1}{2}\left(\frac{\ln x-\mu_{L}}{\sigma_{L}}\right)^{2}} \quad F(x) \stackrel{x \rightarrow 0}{\approx} \kappa_{L} \Phi\left(\frac{\ln x-\mu_{L}}{\sigma_{L}}\right),
$$

where:

$$
\mu_{L}=\ln N+\sigma_{L}^{2} \sum_{i=1}^{N} \frac{\mu_{i}}{\sigma_{i}^{2}}, \quad \kappa_{L}=N \sigma_{L}\left(\prod_{i=1}^{N} \sigma_{i}\right)^{-1} \sqrt{2 \pi}^{1-N} e^{\frac{1}{2} \sigma_{L}^{2}\left(\sum_{i=1}^{N} \frac{\mu_{i}}{\sigma_{i}^{2}}\right)^{2}-\frac{1}{2} \sum_{i=1}^{N} \frac{\mu_{i}^{2}}{\sigma_{i}^{2}}}
$$

\subsubsection{Best Lognormal Fit}

Since the expression in (3.11) is a constant factor of a $\mathrm{LN} p d f$, and assuming the development in Section 3.1.3.1 is valid, we present the following important result:

\section{Hypothesis:}

The SLN has a $b l f \Phi\left(\frac{\ln x-\mu_{L}}{\sigma_{L}}\right)$ at 0 , where the parameters are given by (3.9), (3.12). 


\subsubsection{Difficulties in Approximating the Lower Tail}

It is important to note that, the $\mathrm{LN}$ distribution is very flat near zero; in fact, $\lim _{x \rightarrow 0} \frac{\partial^{n}}{\partial x^{n}} \Phi\left(\frac{\ln x-\mu}{\sigma}\right)=0 \forall n=1,2, \ldots$. The convolution is thus difficult to analyse, as it does not fit in the framework of [55], nor is there a guarantee that the discrete convolution approximation performed in Section 3.1.3.1 is very good. Indeed, we find by simulations that the value of $\kappa_{L}$ does not improve the approximation much, as will be seen in the next section.

\subsubsection{Simulation of lower tail values}

We find that in order to validate our asymptote, it is necessary to simulate the SLN $c d f$ at very low values, which correspond to probabilities as low as $10^{-20}$ or $10^{-30}$, which would require an inversely long simulation length, which is simply prohibitive. We can however make this simulation much more efficient by noticing that the sum of positive RV's is always larger than (or equal to) any of the individual RV's. In other words, we only need to generate low values of $Y_{i}$ in order to obtain low values of the sum $X$. More specifically, to generate the portion of the $c d f$ below a value SimLowVal, we need only generate values of $Y_{i}$ from the distribution $F_{i}$, truncated on the interval $[0$, SimLowVal $]$ and rescaled properly. We can thus obtain the sum distribution using conditional probabilities:

$$
F(x)=P\left(\sum_{i=1}^{N} Y_{i}<x\right)=P\left(\sum_{i=1}^{N} Y_{i}<x \cap\left\{Y_{i}<x \forall i\right\}\right)=P\left(\sum_{i=1}^{N}\left(Y_{i} \mid Y_{i}<x\right)<x\right) \prod_{i=1}^{N} P\left(Y_{i}<x\right)
$$

The algorithm for this simulation is presented as a flowchart in Figure 3-1. There is unfortunately no such simple algorithm for simulating high values of $X$.

In Section 3.1.3.6, we perform some such simulations. The simulations can be done in reasonable time if the number of summands $N$ is not too high, which is why we focused on cases 
of $N=2$, and cases where the summands have different distributions and thus one tends to dominate. In Figures 3-2 - 3-4 we see broken pieces of a curve. Each piece corresponds to one simulation with a different value of SimLowVal. Tail probabilities of the order of $10^{-20}$ can be obtained using only millions of TRIALS (compare with $10^{20}$ ). However, as $N$ increases (say for 6 i.i.d. terms), the algorithm is no longer very efficient.

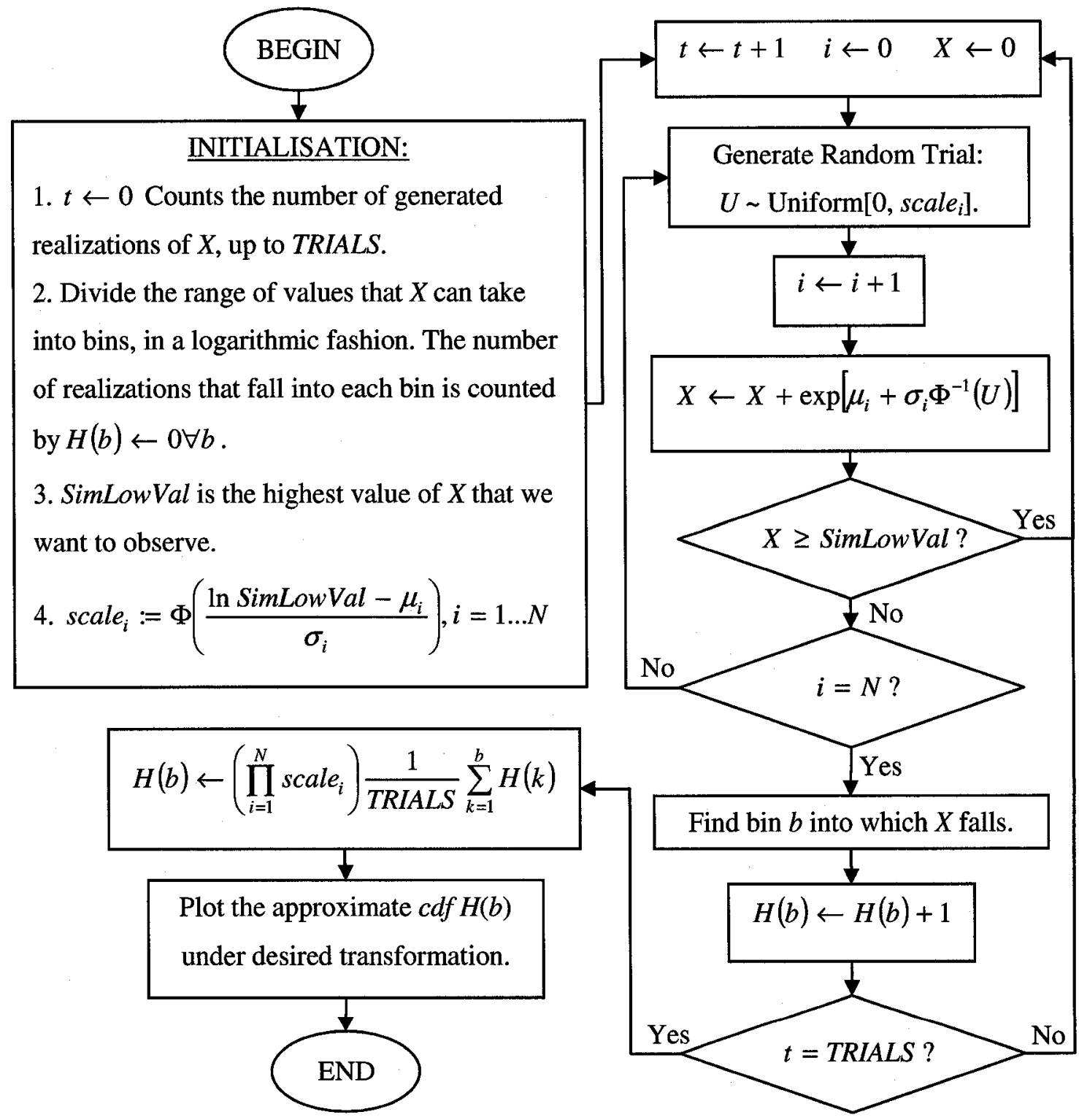

Figure 3-1 Algorithm that efficiently simulates the far lower tail of the SLN cdf. 


\subsubsection{Comparison with Simulations and Recent Methods}

Using the method described in Section 3.1.3.5, we perform Monte-Carlo simulations of the lower tail of the SLN $c d f$ curves, along with our theoretical asymptotes, and we also reproduce some curves from the LL, ZD and BR methods. We plot all these on LN probability paper. The simulations are done using values from Table 3-1 and are shown on Figures 3-2 - 3-4.

We use the values from Table $\mathrm{I}$ in [30] to obtain curves for the BR method for several i.i.d. cases. For the LL curves, we used the values from Table I in [27]. Note that in the fifth row of that Table, the log-variance should be $12 \mathrm{~dB}$, not $6 \mathrm{~dB}$. For the $\mathrm{ZD}$ curves, we used Equations (18)-(20) in [29], which are applicable to i.i.d. cases only, and where $\sigma$ is in dB. The ZS curves are difficult to reproduce because the $c d f$ is not known in closed form [28].

We also plot the theoretical asymptotes: one straight asymptote, the blf $\Phi\left(\frac{\ln x-\mu_{U}}{\sigma_{U}}\right)$, and one LN-times-constant asymptote for each tail (3.11).

We see that in all cases the BR and LL methods, and for most cases, the ZD method, give accurate results in the body of the distribution. However, it is evident from the Figures that each method gives a curve that tends to diverge from the blf of the SLN at either tail. We will show in Section 3.2.1 that this must always be the case. In particular, with the LL method, it could already be seen that there is this divergence in the tails, on LN paper [27].

The SLN converges instead to our proposed asymptotes. We see that the simulated curve tends to be sandwiched between these two curves. By virtue of Lemma 1, this would imply that the SLN $c d f$ is bounded by $\Phi\left(\frac{\ln x-\mu_{U}}{\sigma_{U}}\right)$ from above and by (3.11) from below. Since these two 
functions are constant multiples of each other, it would imply that there exists $\kappa_{L}^{\prime}, \kappa_{L} \leq \kappa_{L}^{\prime} \leq 1$ such that:

$$
\lim _{x \rightarrow 0} \frac{\kappa_{L}^{\prime}}{F(x)} \Phi\left(\frac{\ln x-\mu_{L}}{\sigma_{L}}\right)=1
$$

This would be a much stronger result than simply a blf. However, we cannot prove it.

\begin{tabular}{cccc}
\hline \hline Figure & $\boldsymbol{N}$ & $\boldsymbol{\mu}_{\boldsymbol{i}}(\mathbf{d B})$ & $\boldsymbol{\sigma}_{\boldsymbol{i}}(\mathbf{d B})$ \\
\hline $\mathbf{3 - 2}(\mathbf{A})$ & 2 & 0 & 6 \\
$\mathbf{3 - 2}(\mathbf{B})$ & 2 & 0 & 12 \\
$\mathbf{3 - 3}$ & 6 & 0 & $6,8,9,10,11,12$ \\
$\mathbf{3 - 4}$ & 6 & $-25,-15,-5,5,15,25$ & 12 \\
\hline
\end{tabular}

Table 3-1 Simulation parameters for SLN cdfs in the lower tail.

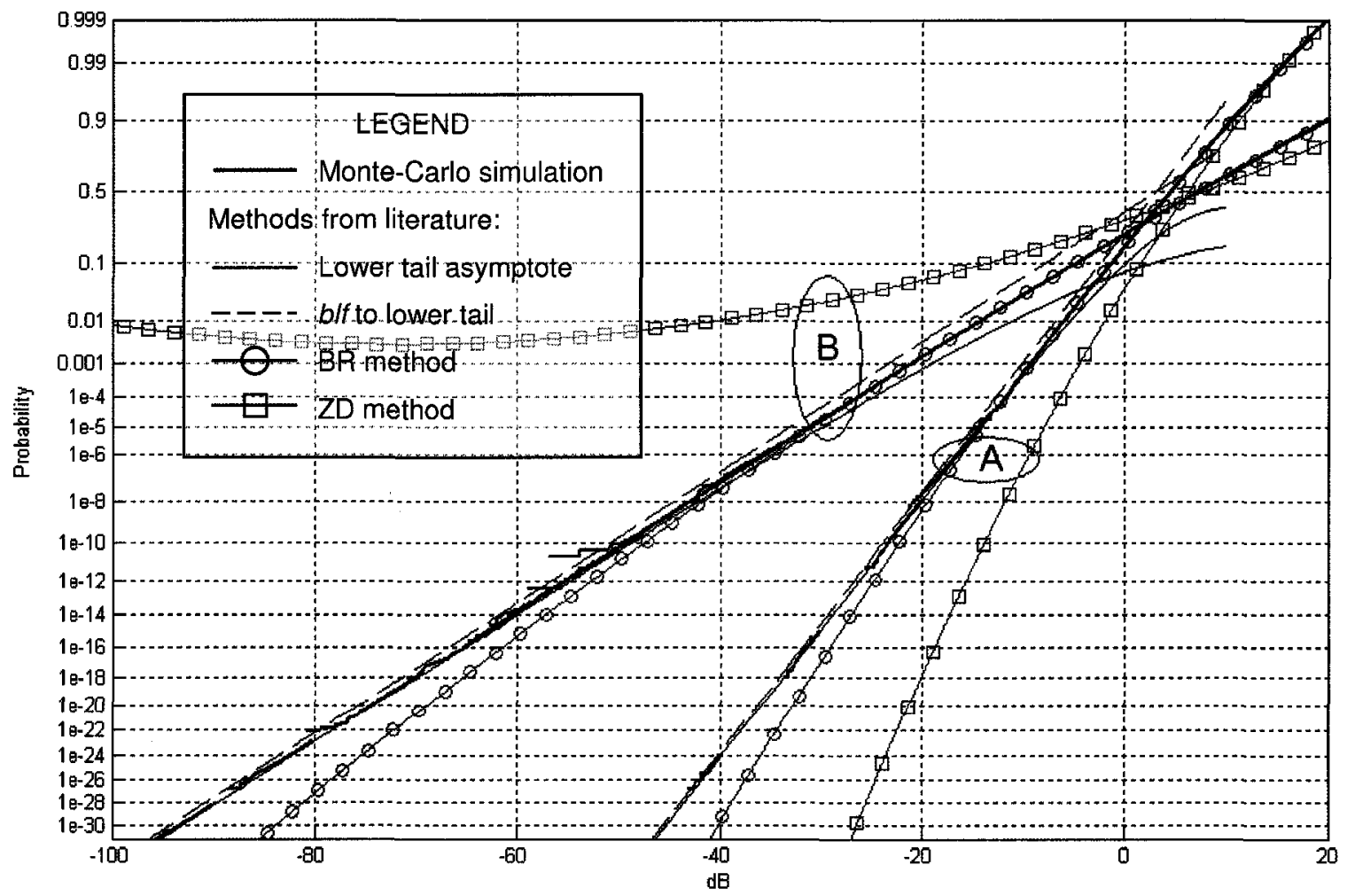

Figure 3-2 The cdf of the sum of two i.i.d. LN RV's $-A: \sigma=6 \mathrm{~dB}$ and $B: \sigma=12 \mathrm{~dB}$. 


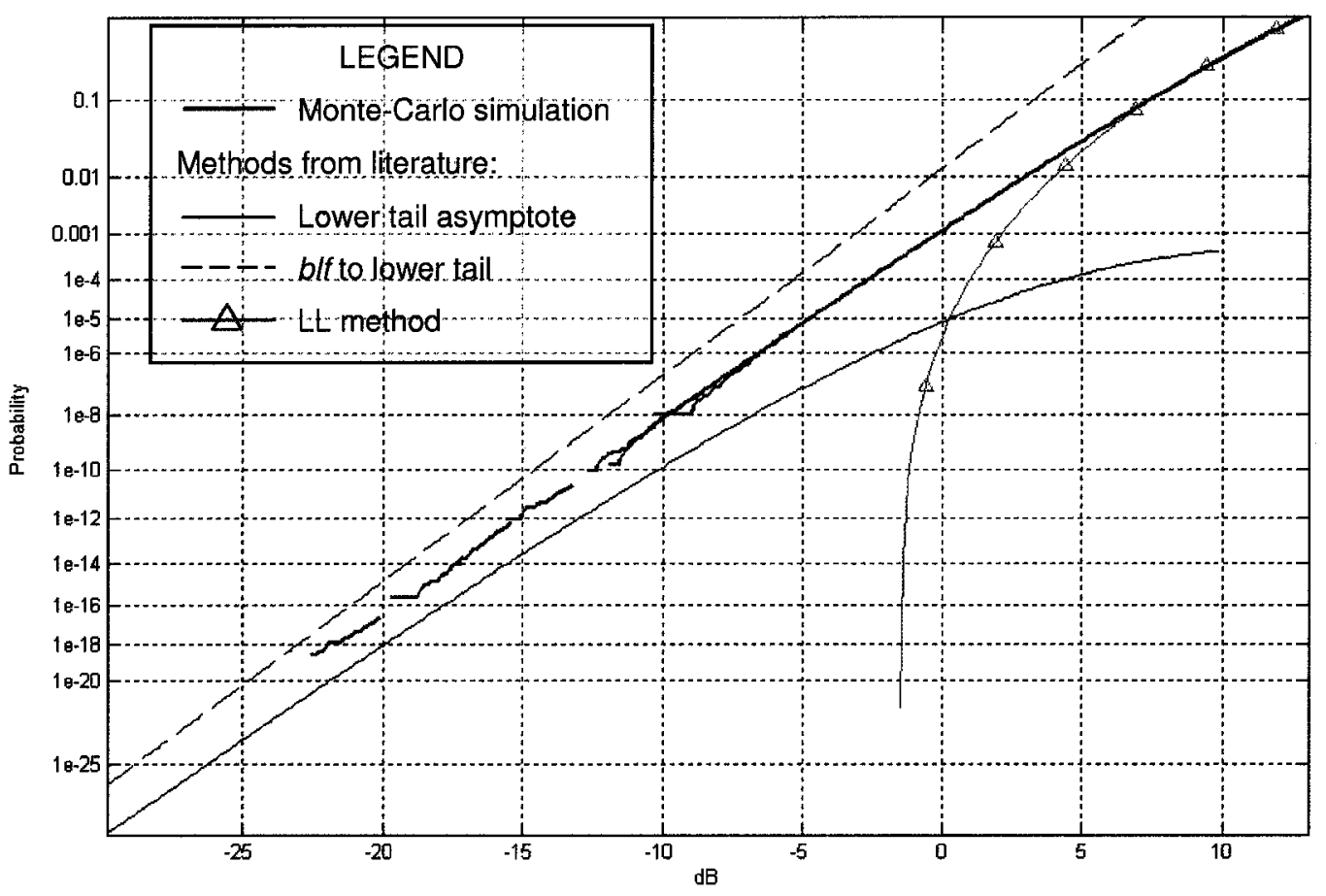

Figure 3-3 The cdf of the sum of lognormals with different variances.

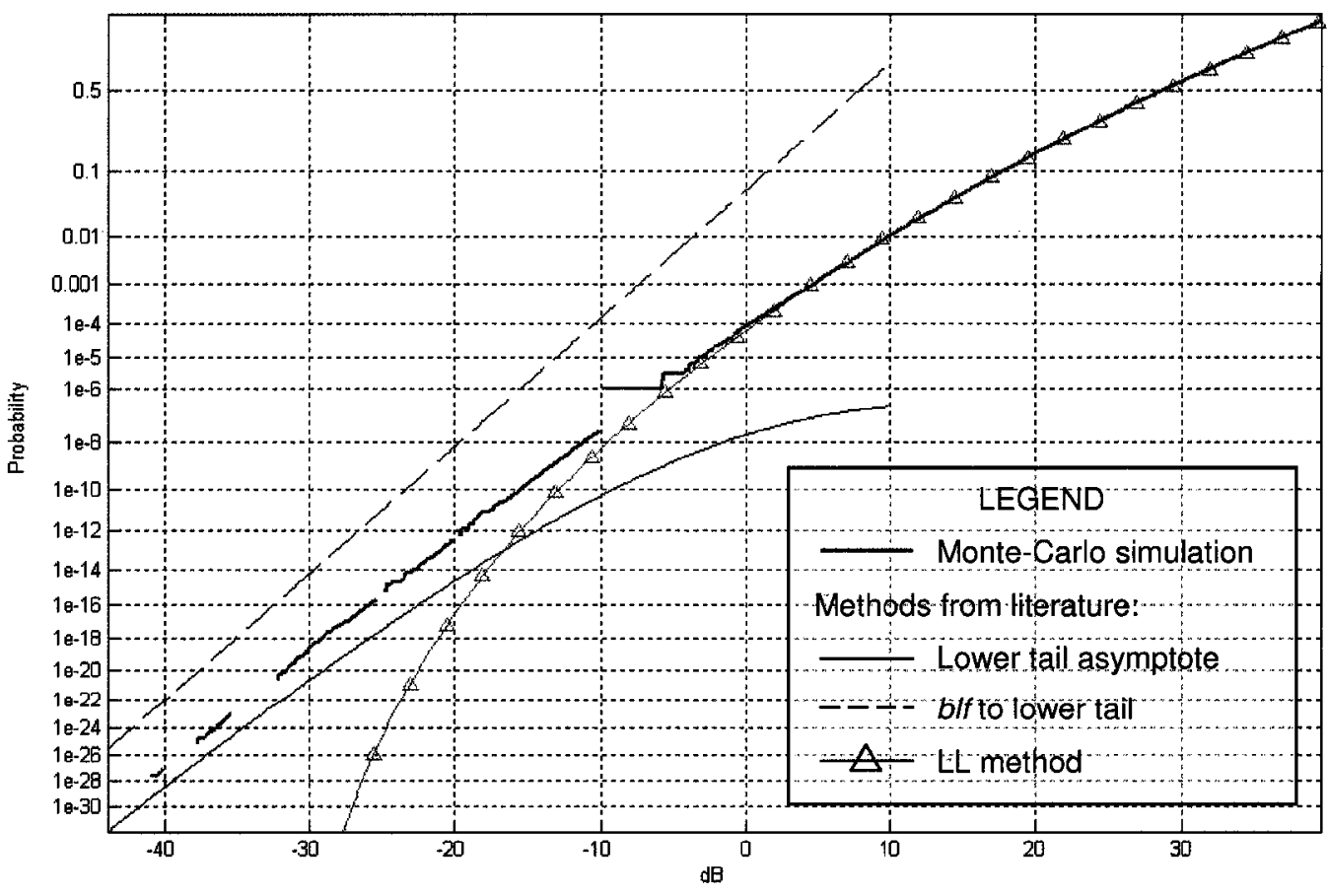

Figure 3-4 The cdf of the sum of lognormals with different means. 


\subsubsection{Upper Tail}

Since the distribution in (2.24), (2.25) is a finite constant multiple of a LN, and Lemma 2 says that if a function is not the blf at a given tail of another function, then the ratio at that tail of those two functions must diverge. Consequently, the SLN distribution has blf $\Phi\left(\frac{\ln x-\mu_{U}}{\sigma_{U}}\right)$ at $+\infty$.

\subsection{Potential Functions for Approximating the SLN Distribution}

Having examined the existing methods for estimating the SLN distribution in Chapter 2, and in light of the results on the tail behaviours of the SLN distribution from Section 3.1, we now show that all the known parametric methods are inadequate in the sense that they necessarily diverge from the true SLN distribution in the tails. We then show how we can construct functions that do respect the tail constraints presented in Sections 3.1.3 and 3.1.4.

\subsubsection{Inadequacy of Existing Functions in the Tails}

Our simulation results suggest that the existing methods for finding the SLN distribution are not accurate in the far tails. The SLN distribution is shown to have a blf in the upper tail, and probably in the lower tail also. In this section, we prove that recent available methods all use distribution functions that cannot have a blf at either tail (except perhaps in the trivial LN case). Consequently, all these methods must inevitably give tail values that have infinite relative error.

We examine four distributions that have recently been proposed as approximations to the SLN. For each of these we show that they cannot possibly give a good approximation at the tails. We do this formally by showing for all four methods that for the proposed $c d f F_{X X}(x), x \geq 0$, for any $\sigma>0, \mu$, the result of the transformation $T: F_{X X}(x) \mapsto \tilde{F}_{X X}(x)$ cannot have $\frac{x-\mu}{\sigma}$ as an asymptote at $l= \pm \infty$, except in some trivial cases when $F_{X X}(x)$ is LN. 
We also show that only some of the proposed functions have the LN distribution as a special case, which should be a characteristic of an SLN approximation.

We use the constant $\lambda=0.1 \ln 10$ to convert from decibel units.

\subsubsection{Log-Shifted Gamma Function: Le-Ngoc - Lam (LL)}

This approximating $c d f$ has three parameters $(\alpha, \beta, \delta)$ [27]:

$$
F_{L L}(x)=\frac{1}{\Gamma(\alpha)} \gamma\left(\frac{\ln x}{\lambda \beta}-\frac{\delta}{\beta}, \alpha\right), x>e^{\delta \lambda}, \beta>0, \alpha>0
$$

where $\gamma(x, \alpha)=\int_{0}^{x} e^{-t} t^{\alpha-1} d t$ is the (lower) incomplete gamma function, and $\Gamma(\alpha)$ is the gamma function.

$$
T: F_{L L}(x) \mapsto \tilde{F}_{L L}(x)=\Phi^{-1}\left(\frac{1}{\Gamma(\alpha)} \gamma\left(\frac{x-\delta \lambda}{\lambda \beta}, \alpha\right)\right), x>\delta \lambda
$$

The function is not even defined for arbitrarily low values.

In the upper tail, we use an approximation of the incomplete gamma function [56]:

$$
\Gamma(\alpha)-\gamma(x, \alpha) \stackrel{x \rightarrow \infty}{\sim} x^{\alpha-1} e^{-x}\left(1+O\left(x^{-1}\right)\right)
$$

and an approximation to the inverse standard normal $c d f$ [57]:

$$
\Phi^{-1}(x)^{x \rightarrow 0} \sim-\sqrt{-2 \ln (2 \sqrt{\pi} x \sqrt{-\ln (2 x)})} .
$$

Then: 


$$
\begin{gathered}
\lim _{x \rightarrow+\infty} \tilde{F}_{L L}(x)-\frac{x-\mu}{\sigma}=\frac{\mu-\lambda \delta}{\sigma}-\lim _{x \rightarrow+\infty} \Phi^{-1}\left(\frac{1}{\Gamma(\alpha)} x^{\alpha-1} e^{-x}\right)-\frac{\lambda \beta}{\sigma} x \\
=\lim _{x \rightarrow+\infty} \sqrt{2 x}-\frac{\lambda \beta}{\sigma} x+\frac{\mu-\lambda \delta}{\sigma}=-\infty
\end{gathered}
$$

Hence, the LL distribution does not have a blf at either tail.

\subsubsection{Three-Parameter Function: Beaulieu - Rajwani (BR)}

This approximating $c d f$ has three parameters $\left(a_{0}, a_{1}, a_{2}\right)$ [30]:

$$
\begin{gathered}
F_{B R}(x)=\Phi\left(a_{0}-a_{1} x^{-\frac{a_{2}}{\lambda}}\right), x \geq 0, a_{0}>0, a_{1}>0, a_{2}>0, \\
T: F_{B R}(x) \mapsto \tilde{F}_{B R}(x)=a_{0}-a_{1} e^{-\frac{a_{2}}{\lambda} x} .
\end{gathered}
$$

Then:

$$
\lim _{x \rightarrow l} \tilde{F}_{B R}(x)-\frac{x-\mu}{\sigma}=a_{0}+\frac{\mu}{\sigma}-\lim _{x \rightarrow l} a_{1} e^{-\frac{a_{2}}{\lambda} x}+\frac{x}{\sigma}= \pm \infty
$$

Hence, the BR distribution does not have a blf at either tail.

In [29] it is mentioned that this form does not include the $\mathrm{LN}$ distribution as a special case. We can, however, obtain convergence of $F_{B R}(x) \rightarrow \Phi\left(\frac{\ln x-\mu}{\sigma}\right) \forall x$ :

Let us consider $\mu, \sigma$ as constants, such that:

$$
\mu=\frac{a_{1}-a_{0}}{a_{1} a_{2}}, \sigma=\frac{1}{a_{1} a_{2}}
$$

Then: 


$$
F_{B R}(x)=\Phi\left(\frac{-\frac{a_{1}-a_{0}}{a_{1} a_{2}}-\left(\frac{x^{-a_{2}}-1}{a_{2}}\right)}{\frac{1}{a_{1} a_{2}}}\right)=\Phi\left(\frac{\left(\frac{1-x^{-a_{2}}}{a_{2}}\right)-\mu}{\sigma}\right)
$$

Using an alternative definition of the natural logarithm:

$$
\ln x=\lim _{a \rightarrow 0} \frac{1-x^{-a}}{a}, x \geq 0
$$

we obtain:

$$
F_{B R}(x) \stackrel{a_{2} \rightarrow 0}{\longrightarrow} \Phi\left(\frac{\ln x-\mu}{\sigma}\right), x \geq 0
$$

Thus, in the limiting case:

$$
a_{2} \rightarrow 0, a_{1}=\left(a_{2} \sigma\right)^{-1}, a_{0}=a_{1}-\mu / \sigma,
$$

the BR distribution is LN.

\subsubsection{Three-Parameter Function: Zhao - Ding (ZD)}

This approximating $c d f$ has three parameters $\left(c_{0}, c_{1}, c_{2}\right)$ [29]:

$$
\begin{gathered}
F_{Z D}(x)=\Phi\left(c_{0}+c_{1} \frac{\ln x}{\lambda}+c_{2}\left(\frac{\ln x}{\lambda}\right)^{2}\right), x \geq 0, \\
T: F_{Z D}(x) \mapsto \tilde{F}_{Z D}(x)=c_{0}+\frac{c_{1}}{\lambda} x+\frac{c_{2}}{\lambda^{2}} x^{2} .
\end{gathered}
$$

Then, if $c_{2} \neq 0$ :

$$
\lim _{x \rightarrow l} \tilde{F}_{Z D}(x)-\frac{x-\mu}{\sigma}=\lim _{x \rightarrow l} \frac{c_{2}}{\lambda^{2}} x^{2}+\left(\frac{c_{1}}{\lambda}-\frac{1}{\sigma}\right) x+c_{0}+\frac{\mu}{\sigma}= \pm \infty
$$

Hence, the ZD distribution does not have a $b l f$ at either tail, except in the LN case $c_{2}=0$. 


\subsubsection{Pearson Type IV Function: Zhang - Song (ZS)}

The proposed distribution for the SLN in $\mathrm{dB}$ is Pearson type IV, which has four parameters $(u, m, d, v)$ [28]. Consequently, the $p d f$ of the SLN in linear scale has distribution:

$$
\begin{gathered}
f_{Z S}(x)=\frac{k}{\lambda x}\left(1+y^{2}\right)^{-m} e^{-v \arctan y}, \\
y=\frac{\ln x}{\lambda d}+\frac{u}{d}, m>\frac{1}{2}, x \geq 0,
\end{gathered}
$$

and $k$ is the constant chosen so that the $p d f$ be valid and is found by numerical integration for each particular case. It would seem that the $c d f$ is not readily available in closed form. However, we can still study its tail behaviour:

$$
\begin{aligned}
f_{Z S}(x) \stackrel{x \rightarrow 0}{\sim} & k|d|^{2 m} \lambda^{2 m-1} x^{-1}|\ln x|^{-2 m} e^{v \frac{\pi}{2} \operatorname{sgn}(d)} \\
& =C_{1} \frac{|\ln x|^{-2 m}}{x}, C_{1}>0, \\
F_{Z S}(x) \stackrel{x \rightarrow 0}{\sim} & =\int_{\zeta}^{x} C_{1} \frac{|\ln y|^{-2 m}}{y} d y=C_{1} \frac{|\ln x|^{1-2 m}}{2 m-1} .
\end{aligned}
$$

Again, using the result (3.18):

$$
\begin{gathered}
T: F_{Z S}(x) \mapsto \tilde{F}_{Z S}(x) \stackrel{x \rightarrow-\infty}{\sim} \Phi^{-1}\left(\frac{C_{1}}{2 m-1}|x|^{1-2 m}\right) \\
\stackrel{x \rightarrow-\infty}{\sim}-\sqrt{(4 m-2)} \sqrt{\ln (-x)} \Rightarrow \lim _{x \rightarrow-\infty} \tilde{F}_{Z D}(x)-\frac{x-\mu}{\sigma} \\
\quad=-\lim _{x \rightarrow-\infty} \sqrt{(4 m-2)} \sqrt{\ln (-x)}+\frac{x-\mu}{\sigma}=+\infty .
\end{gathered}
$$

A similar analysis applies for the upper tail.

Hence, the ZS distribution does not have a blf at either tail. 


\subsubsection{Potential Function Candidates}

If the existing methods use $c d f$ 's that cannot behave properly in the tails, what then would functions that do look like? We give here a collection of three functions that verify at least some of the tail requirements given in Sections 3.1.3.2 and 2.2.2.

\subsubsection{Power Lognormal}

A PLN $c d f$ has general form:

$$
F_{P L N}(x)=\Phi^{t}\left(\frac{\ln x-m}{s}\right), x \geq 0 \quad t \geq 1, s>0
$$

It has $p d f$ :

$$
f_{P L N}(x)=\frac{t}{\sqrt{2 \pi} x s} \exp \left[-\frac{1}{2}\left(\frac{\ln x-m}{s}\right)^{2}\right] \Phi^{t-1}\left(\frac{\ln x-m}{s}\right), x \geq 0
$$

It has blf $\Phi\left(\frac{\ln x-m}{s}\right)$ at $+\infty$. Proof:

$$
\lim _{x \rightarrow+\infty} \frac{1-F_{P L N}(x)}{1-\Phi\left(\frac{\ln x-m}{s}\right)}=\lim _{x \rightarrow+\infty} \frac{f_{P L N}(x)}{\frac{1}{\sqrt{2 \pi} x s} \exp \left[-\frac{1}{2}\left(\frac{\ln x-m}{s}\right)^{2}\right]}=\lim _{x \rightarrow+\infty} t \Phi^{t-1}\left(\frac{\ln x-m}{s}\right)=t \text { (3.37) }
$$

By lemma 2, since the limit is finite (non-zero), the function is indeed the blf. This completes the proof.

The lower tail poses more of a problem, because it does not have a $b l f$ unless $t=1$.

Proof:

Consider 


$$
T: F_{P L N}(x) \mapsto \tilde{F}_{P L N}(x)=\Phi^{-1}\left(\Phi^{t}\left(\frac{x-m}{s}\right)\right)
$$

Using the following approximation:

$$
\Phi(x) \stackrel{x \rightarrow-\infty}{\sim} \frac{1}{-\sqrt{2 \pi} x} e^{-\frac{1}{2} x^{2}}
$$

we find:

$$
\tilde{F}_{P L N}(x) \stackrel{x \rightarrow-\infty}{\sim} \Phi^{-1}\left(\frac{s^{t} e^{-\frac{t}{2}\left(\frac{x-m}{s}\right)^{2}}}{\sqrt{2 \pi}^{t}(m-x)^{t}}\right)
$$

We use the tail approximation (3.18) from [57]:

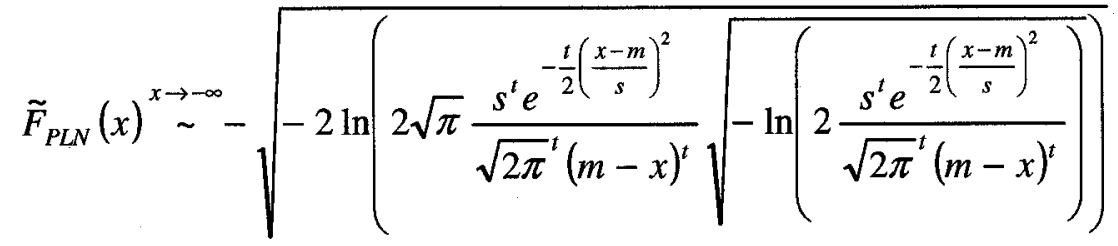

$$
\begin{aligned}
& =-\sqrt{-2 t \ln \left(2 \sqrt{\pi} \frac{s}{\sqrt{2 \pi}}\right)+t\left(\frac{x-m}{s}\right)^{2}+2 t \ln (m-x)}-\sqrt{-2 \ln \left(\sqrt{-t \ln \left(2 \frac{s}{\sqrt{2 \pi}}\right)+t \ln (m-x)+\frac{t}{2}\left(\frac{x-m}{s}\right)^{2}}\right)} \\
& =-\sqrt{-2 t \ln \left(2 \frac{s}{\sqrt{2 \pi}}\right)+t\left(\frac{x-m}{s}\right)^{2}-\ln \left(\frac{-t \ln \left(2 \frac{s}{\sqrt{2 \pi}}\right)+t \ln (m-x)}{(m-x)^{2 t}}+\frac{t(m-x)^{2(1-t)}}{2 s^{2}}\right)} \\
& \sim-\sqrt{t\left(\frac{x-m}{s}\right)^{2}-2 \ln \left(\frac{\sqrt{t}(m-x)^{(1-t)}}{\sqrt{2} s}\right)} \sim-\sqrt{t\left(\frac{x-m}{s}\right)^{2}+2(t-1) \ln (-x)} .
\end{aligned}
$$

Now if $\Phi\left(\frac{\ln x-\mu}{\sigma}\right)$ is the blf at 0 , then the following equation must hold: 


$$
\begin{gathered}
\exists \mu, \exists \sigma: \lim _{x \rightarrow-\infty} \frac{x-\mu}{\sigma}+\sqrt{t\left(\frac{x-m}{s}\right)^{2}+2(t-1) \ln (-x)}=0 \\
\Rightarrow \lim _{x \rightarrow-\infty}\left(\frac{x-\mu}{\sigma}\right)^{2}+t\left(\frac{x-m}{s}\right)^{2}+2(t-1) \ln (-x)+2\left(\frac{x-\mu}{\sigma}\right) \sqrt{t\left(\frac{x-m}{s}\right)^{2}+2(t-1) \ln (-x)}=0 \\
\Rightarrow \lim _{x \rightarrow-\infty}\left(\frac{x-\mu}{\sigma}\right)^{2}+t\left(\frac{x-m}{s}\right)^{2}+2(t-1) \ln (-x)-2 \sqrt{t}\left(\frac{x-\mu}{\sigma}\right)\left(\frac{x-m}{s}\right)=0 \\
\Rightarrow \lim _{x \rightarrow-\infty} A x^{2}+B x+C+2(t-1) \ln (-x)=0 .
\end{gathered}
$$

This has no solutions, for any constants $A, B, C$, unless $t=1$. This proves that the PLN $c d f$ does not have a blf at $-\infty$. However the lower tail does have a limiting slope:

$$
\begin{gathered}
\lim _{x \rightarrow-\infty} \frac{\partial}{\partial x} \tilde{F}_{P L N}(x)=-\lim _{x \rightarrow-\infty} \frac{\partial}{\partial x} \sqrt{t\left(\frac{x-m}{s}\right)^{2}+2(t-1) \ln (-x)} \\
=-\lim _{x \rightarrow-\infty} \frac{2 \frac{t}{s}\left(\frac{x-m}{s}\right)-\frac{2(t-1)}{x}}{2 \sqrt{t\left(\frac{x-m}{s}\right)^{2}+2(t-1) \ln (-x)}}=\lim _{x \rightarrow-\infty} \frac{2 \frac{t}{s}\left(\frac{x-m}{s}\right)-\frac{2(t-1)}{x}}{2 x \sqrt{t\left(\frac{1}{s}-\frac{m}{s x}\right)^{2}+2(t-1) \frac{\ln (-x)}{x^{2}}}} \\
=\lim _{x \rightarrow-\infty} \frac{2 \frac{t}{s}\left(\frac{x-m}{s}\right)}{2 x \sqrt{\left(\frac{x-m}{s x}\right)^{2}}}=\lim _{x \rightarrow-\infty} \frac{t\left(\frac{x-m}{s}\right)}{-s \sqrt{t}\left(\frac{m-x}{s}\right)}=\frac{\sqrt{t}}{s} .
\end{gathered}
$$

We are thus interested in the PLN distribution because it has a blf in the upper tail, and at least a limiting slope in the lower tail. Also, [22] suggested that the SLN distribution could be written as the sum of products of LN distributions. A PLN distribution is a particular case of this, which makes it an interesting candidate.

\subsubsection{A Four-Parameter Function}

Since we have seen that the SLN has $b l f^{\prime}$ s at both tails, it would be interesting to create a function that also has the same $b l f^{\prime} \mathrm{s}$ at both tails. To do this, we first construct a function $r(x)$, such that: 
1. $r(x)$ is analytically smooth.

2. $\lim _{x \rightarrow-\infty} r(x)=0$.

3. $\lim _{x \rightarrow+\infty} r(x)-x=k$.

One such function is:

$$
r(x, k)=\ln \left(1+e^{k} e^{x}\right)
$$

Now, in order to construct an approximation to the SLN $c d f$, we construct the following fourparameter (4P) function:

$$
\tilde{F}_{4 P}(x)=a x-r\left(c x, \frac{d}{b}\right)-\ln b=a x-\ln \left(b+d e^{c x}\right), a>c>0,
$$

which has tail behaviour:

$$
\tilde{F}_{4 P}(x) \stackrel{x \rightarrow+\infty}{\approx}(a-c) x-\ln d \quad \text { and } \quad \tilde{F}_{4 P}(x) \stackrel{x \rightarrow-\infty}{\approx} a x-\ln b .
$$

Consequently,

$$
T^{-1}: \tilde{F}_{4 P}(x) \mapsto F_{4 P}(x)=a x-r\left(c x, \frac{d}{b}\right)-\ln b=\Phi\left(\ln \left(\frac{x^{a}}{b+d x^{c}}\right)\right), a>c>0
$$

is our proposed function 4P for approximating SLN. It has blf $\Phi((a-c) \ln x-\ln d)$ at $+\infty$, and blf $\Phi(a \ln x-\ln b)$ at $-\infty$.

\subsection{Obtaining the Parameters of the Approximating Functions}

\subsubsection{Power Lognormal Distribution}

We wish to fit the PLN $c d f$ to that of the SLN as well as possible. We know the blf of both the upper and lower tail of the SLN, and we can also find its moments analytically, whereas the PLN 
distribution has three free parameters only. We choose to match the slopes of both tails first, because an error in the slope of the tail asymptote will become infinitely more significant than an error in the offset as we approach the limit. We also found that matching the first moment of the PLN $c d f$ with that of the SLN was a best-effort method to get a good curve match. It is fortunate that we do not have to solve for all three parameters simultaneously, but rather we first find $s$, then, using $s$, find $t$, finally we find $m$ using a numerical integral involving $s$ and $t$.

\subsubsection{Matching the Tail Slopes}

We first find the value of $s$. The upper tail slope is given by the blf at $+\infty$ (see Sections 3.1.4 and (2.21)) and is matched with the blf at $+\infty$ of the PLN, given in Section 3.2.2.1 :

$$
s=\sigma_{U}=\max _{i} \sigma_{i}
$$

From the value of $s$ we can find $t$. The lower tail slope is given by the blf at $-\infty$ (3.9) and is matched with (3.43):

$$
\frac{\sqrt{t}}{s}=\frac{\sqrt{t}}{\sigma_{U}}=\frac{1}{\sigma_{L}}=\left(\sum_{i=1}^{N} \frac{1}{\sigma_{i}^{2}}\right)^{\frac{1}{2}} \Rightarrow t=\sigma_{U}^{2} \sum_{i=1}^{N} \frac{1}{\sigma_{i}^{2}}=\sum_{i=1}^{N} \frac{\max _{i} \sigma_{i}^{2}}{\sigma_{i}^{2}} \geq N,
$$

and $t=N$ if and only if all variances are equal.

\subsubsection{Moment-Matching}

The moments of the of the PLN distribution with pdf (3.36) are: 


$$
\begin{gathered}
E\left[X^{n}\right]=\frac{t}{\sqrt{2 \pi} s} \int_{0}^{+\infty} x^{n} \exp \left[-\frac{1}{2}\left(\frac{\ln x-m}{s}\right)^{2}\right] \Phi^{t-1}\left(\frac{\ln x-m}{s}\right) \frac{d x}{x} \\
=\frac{t}{\sqrt{2 \pi} s} \int_{-\infty}^{+\infty} e^{x n} \exp \left[-\frac{1}{2}\left(\frac{x-m}{s}\right)^{2}\right] \Phi^{t-1}\left(\frac{x-m}{s}\right) d x \\
=\frac{t}{\sqrt{2 \pi} s} e^{m n} \int_{-\infty}^{+\infty} e^{x n} \exp \left[-\frac{1}{2}\left(\frac{x}{s}\right)^{2}\right] \Phi^{t-1}\left(\frac{x}{s}\right) d x \\
=e^{m n} \Lambda(s n, t), \quad \Lambda(s n, t)=\frac{t}{\sqrt{2 \pi}} \int_{-\infty}^{+\infty} e^{x s n-\frac{1}{2} x^{2}} \Phi^{t-1}(x) d x,
\end{gathered}
$$

where the integral $\Lambda$ needs to be calculated numerically as a function of $s$ and $t$ found in Section 3.3.1.1. This is essentially the same expression as found in [58], where the PLN distribution is defined slightly differently. Notice that the parameter $m$ comes out of the integral, which allows us to solve for $m$, using the first moment:

$$
m=\ln E[X]-\ln \Lambda(s, t)=\ln \sum_{i=1}^{N} e^{\mu_{i}+\frac{1}{2} \sigma_{i}^{2}}-\ln \Lambda(s, t)
$$

Although our method requires numerical integration at this point, it still remains much simpler than other numerical methods, for the following reasons:

1. The integration needs only be performed once, not iteratively for all summands as in SY [37] or LL [27].

2. The integral is very well-behaved and can be calculated directly using a Riemann sum, unlike some of the numerical methods used in other SLN methods [35].

We calculate the integral by summing it over the interval $[-25,25]$ with step 0.01 .

We can partially verify our method since we do have a closed-form expression for the moments of the PLN distribution when $t=2$ : 


$$
\begin{gathered}
\Lambda(s n, 2)=\frac{2}{\sqrt{2 \pi}} \int_{-\infty}^{+\infty} e^{x s n-\frac{1}{2} x^{2}} \Phi(x) d x \\
=\frac{2}{2 \pi} \int_{-\infty}^{+\infty} e^{x s n-\frac{1}{2} x^{2}} \int_{-\infty}^{x} e^{-\frac{1}{2} t^{2}} d t d x=\frac{1}{\pi} \int_{-\infty}^{+\infty} \int_{-\infty}^{x} e^{x s n-\frac{1}{2} x^{2}-\frac{1}{2} t^{2}} d t d x .
\end{gathered}
$$

Now make the change of variables:

$$
\begin{gathered}
\left\{\begin{array} { l } 
{ a = x + t } \\
{ b = x - t }
\end{array} \Rightarrow \left\{\begin{array}{l}
x=\frac{a+b}{2} \\
t=\frac{a-b}{2}
\end{array} \Rightarrow J=\frac{\partial(x, t)}{\partial(a, b)}=-\frac{1}{2} \Rightarrow|J|=\frac{1}{2} .\right.\right. \\
E\left[X^{n} \mid t=2\right]=e^{m n} \Lambda(s n, 2)=\frac{e^{m n}}{2 \pi} \int_{-\infty}^{+\infty} \int_{0}^{+\infty} e^{-\frac{1}{4}\left(a^{2}+b^{2}\right)+\frac{s n}{2}(a+b)} d b d a \\
=\frac{e^{m n}}{2 \pi} \int_{-\infty}^{+\infty} e^{-\frac{1}{4} x^{2}+\frac{s n}{2} x} d x \int_{0}^{+\infty} e^{-\frac{1}{4} x^{2}+\frac{s n}{2} x} d x=\frac{e^{m n}}{\sqrt{\pi}} \sqrt{2 \pi} \int_{0}^{+\infty} e^{-\frac{1}{2} x^{2}+\frac{s n}{\sqrt{2}} x} d x=2 \Phi\left(\frac{s n}{\sqrt{2}}\right) e^{m n+\frac{1}{2} s^{2} n^{2}} .
\end{gathered}
$$

We verify this by comparing with numerical values in Table 3-2, where we see that the Riemann sum and the theoretical values coincide perfectly. However, we were not able to do a separation of variables for $t$ higher than 2, so the general closed-form expression for the moments of a PLN distribution is to our knowledge unknown.

\begin{tabular}{ccc}
\hline \hline$s n$ & $E\left[X^{n} \mid t=2, m=0\right]=2 \Phi\left(\frac{s n}{\sqrt{2}}\right) e^{\frac{1}{2} s^{2} n^{2}}$ & Numerical $\Lambda(s n, 2)$ \\
\hline 1.38155 & $4.3405163407083 \underline{4}$ & $4.3405163407083 \underline{\underline{2}}$ \\
2.76310 & $88.661408234589 \underline{91}$ & $88.661408234589 \underline{83}$ \\
5.52620 & $8559626.6550080 \underline{20}$ & $8559626.6550080 \underline{11}$ \\
11.0524 & $6.7113558551748 \underline{30} \cdot 10^{26}$ & $6.7113558551748 \underline{11} \cdot 10^{26}$ \\
22.1048 & $2.53 \underline{6010695502967} \cdot 10^{106}$ & $2.53 \underline{1281918731208 \cdot 10^{106}}$ \\
\hline \hline
\end{tabular}

Table 3-2 Analytical and numerical calculations of moments of the PLN distribution when the power is $t=2$, with differences underlined. 


\subsubsection{Four-Parameter Function}

Based on the blf's given in Section 3.2.2.2, the system of equations for matching asymptotes is:

$$
\frac{1}{\sigma_{U}}=a-c, \quad \frac{\mu_{U}}{\sigma_{U}}=\ln d, \quad \frac{1}{\sigma_{L}}=a, \quad \frac{\mu_{L}}{\sigma_{L}}=\ln b .
$$

This is directly solvable:

$$
a=\frac{1}{\sigma_{L}}, \quad b=e^{\frac{\mu_{L}}{\sigma_{L}}}, \quad c=\frac{1}{\sigma_{L}}-\frac{1}{\sigma_{U}}, \quad d=e^{\frac{\mu_{U}}{\sigma_{U}}},
$$

where the parameters are given by equations (2.21), (2.22), (3.9), and (3.12). After substitutions, we obtain the following closed-form expression in terms of the parameters of the summands:

$$
F_{4 P}(x)=\Phi\left(\frac{\ln x-\mu_{U}}{\sigma_{U}}-\ln \left(1+e^{\frac{\ln x-\mu_{U}}{\sigma_{U}}-\frac{\ln x-\mu_{L}}{\sigma_{L}}}\right)\right)
$$

\subsubsection{Evaluation of Closed-Form Methods against Simulations}

In this section, we evaluate the performance of the two SLN methods we developed against all other known closed-form methods. The known closed-form methods are:

1. Moment-Matching by the FW method using (2.16)-(2.19). We only consider matching the first and second moment, as these give the best results overall [40].

2. The lower bound expression in (2.26), which is an upper bound to the $c d f$.

3. The upper bound expression in (2.28), which is a lower bound to the $c d f$.

4. The AG method given by (2.30), (2.33).

We also plot our two new methods: PLN and 4P. While our PLN method is not strictly closedform, the numerical integration required is minimal (and there is hope that the numerical integral in (3.50) can some day be found in closed form, at least for integer $t$ ). We run Monte-Carlo 
simulations to validate all these methods. We have chosen four cases, described in Table 3-3.

The results are plotted on LN paper in Figures 3-5 - 3-8.

\begin{tabular}{cccc}
\hline \hline Figure & $\boldsymbol{N}$ & $\boldsymbol{\mu}_{\boldsymbol{i}}$ (natural units) & $\boldsymbol{\sigma}_{\boldsymbol{i}}(\mathbf{d B})$ \\
\hline $\mathbf{3 - 5}$ & 6 & 0 & 12 \\
$\mathbf{3 - 6}$ & 30 & 0 & 6 \\
& & $0,1,2,3,4,5$ & \\
$\mathbf{3 - 7}$ & 12 & $6,7,8,9,10,11$ & \\
& & $5,5,5,5,5,5$, & $12,12,12,12,12,12$, \\
& & $4,4,4,4,4,4$, & $11,11,11,11,11,11$, \\
& & $3,3,3,3,3,3$, & $10,10,10,10,10,10$, \\
$3-8$ & 36 & $2,2,2,2,2,2$, & $9,9,9,9,9,9$, \\
& & $1,1,1,1,1,1$, & $8,8,8,8,8,8$, \\
& & $0,0,0,0,0,0$ & $7,7,7,7,7,7$ \\
\hline
\end{tabular}

Table 3-3 Simulation parameters for sums of lognormals.

From the simulation results, we make the following observations:

1. The FW method performs well in approximating the upper tail.

2. The true SLN $c d f$ is sandwiched by the two bounds (2.26) and (2.28).

3. The AG method gives a rather loose bound, as predicted in Section 2.2.3.2.

4. Our PLN method tends to perform better than all other methods, particularly in the complicated case of Figure 3-8. In particular, it performs better than the upper and lower bounds, which are also of the form of a product of $N \mathrm{LN} c d f$ s.

5. Our 4P method does not give a very close match to the real SLN $c d f$, particularly in the body. However, with additional degrees of freedom, we believe that we can make the curve move in the body so as to fit the entire curve well. Also, we conjecture that the $4 \mathrm{P}$ $c d f$ is an upper bound to the SLN $c d f$.

In light of these observations, we choose both the FW method and our PLN method as candidates to approximate the interference distribution. The FW method is commonly used in similar interference analysis work [9], [13], [16], and is thus a good benchmark for comparison. Both methods are simple to implement, as will be seen in Chapter 4 . 


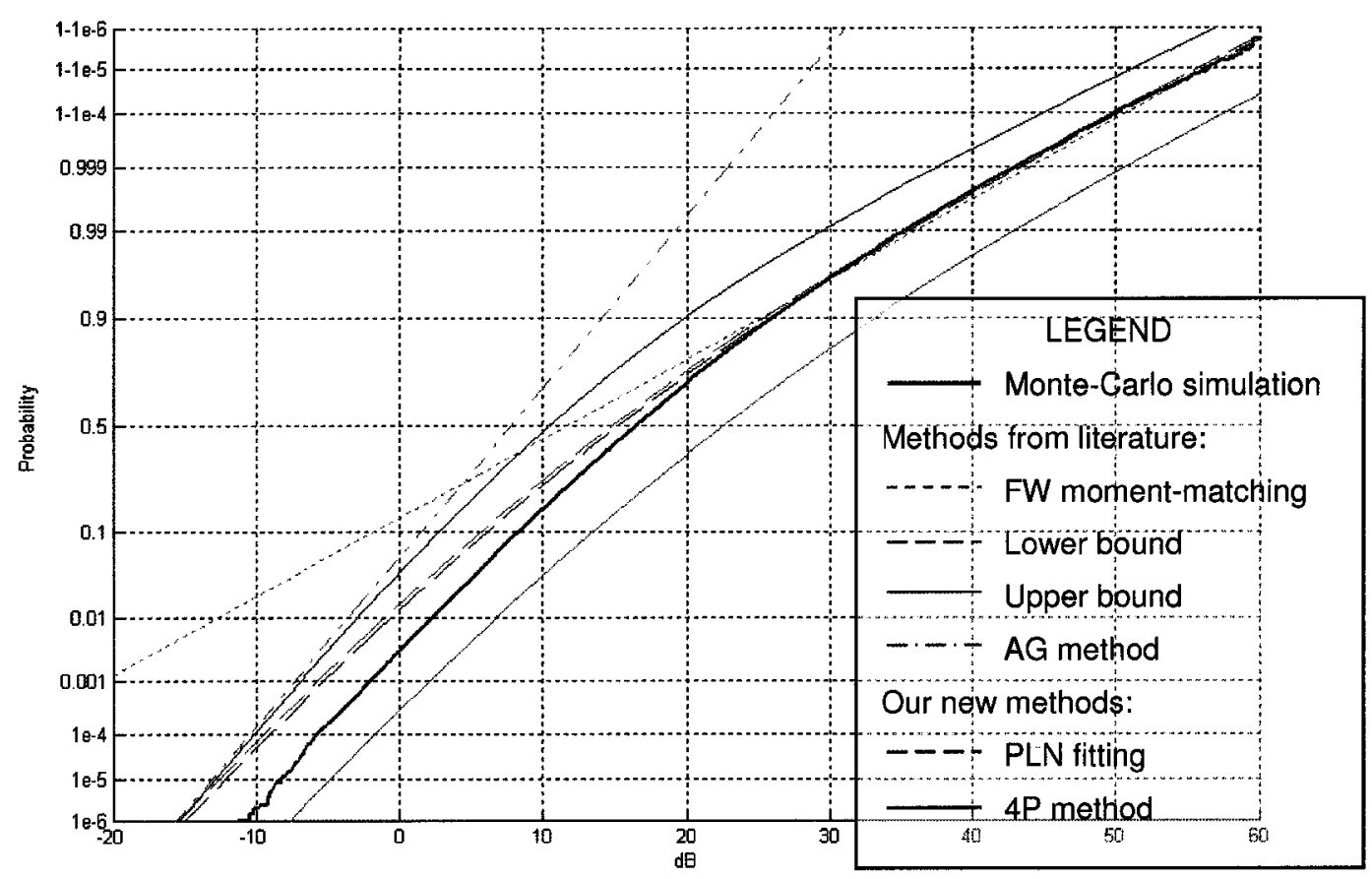

Figure 3-5 The cdf of the sum of 6 i.i.d. lognormals, and closed-form methods.

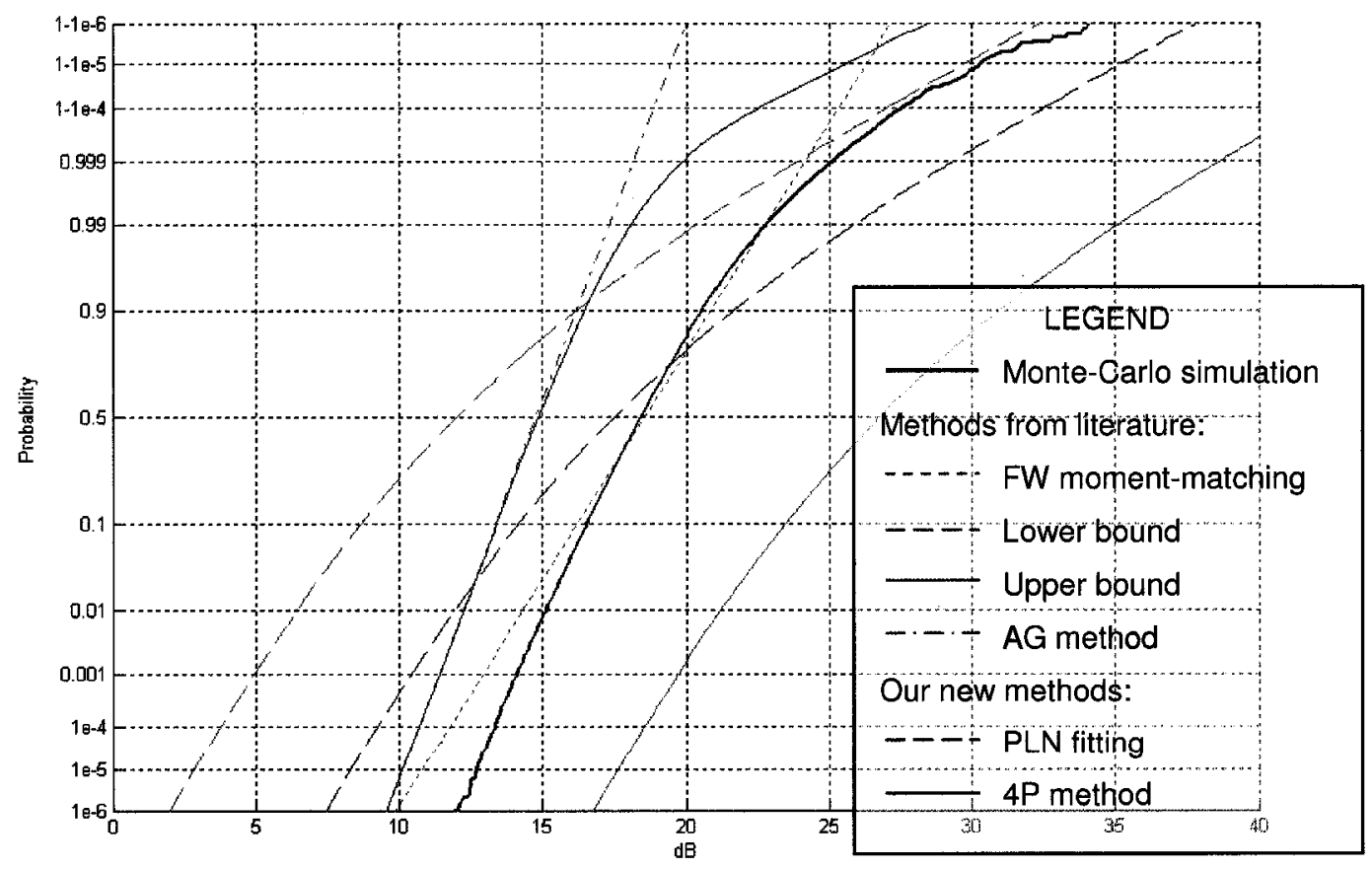

Figure 3-6 The cdf of the sum of 30 i.i.d. lognormals, and closed-form methods. 


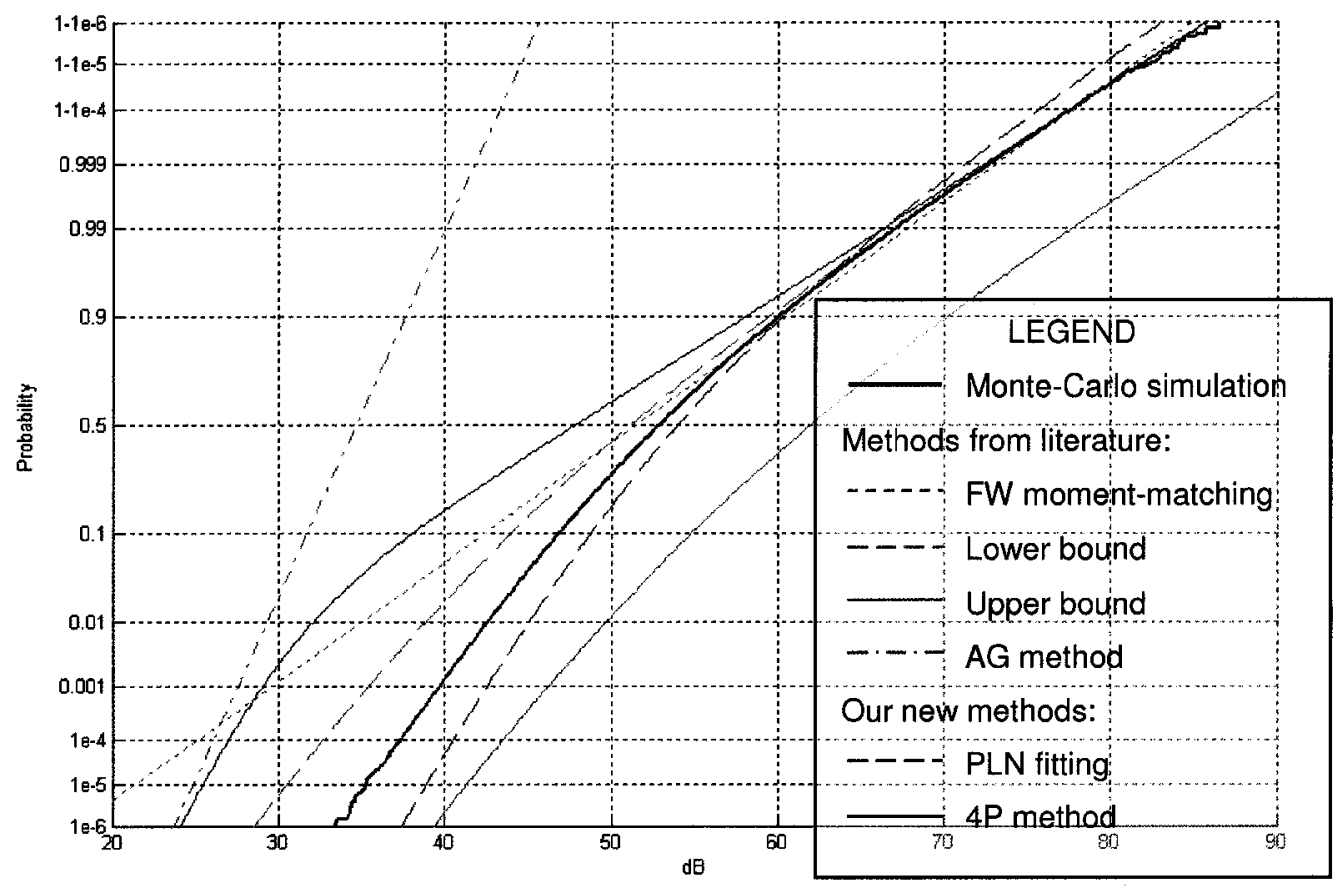

Figure 3-7 The cdf of the sum of 12 lognormals with different means, and closedform methods.

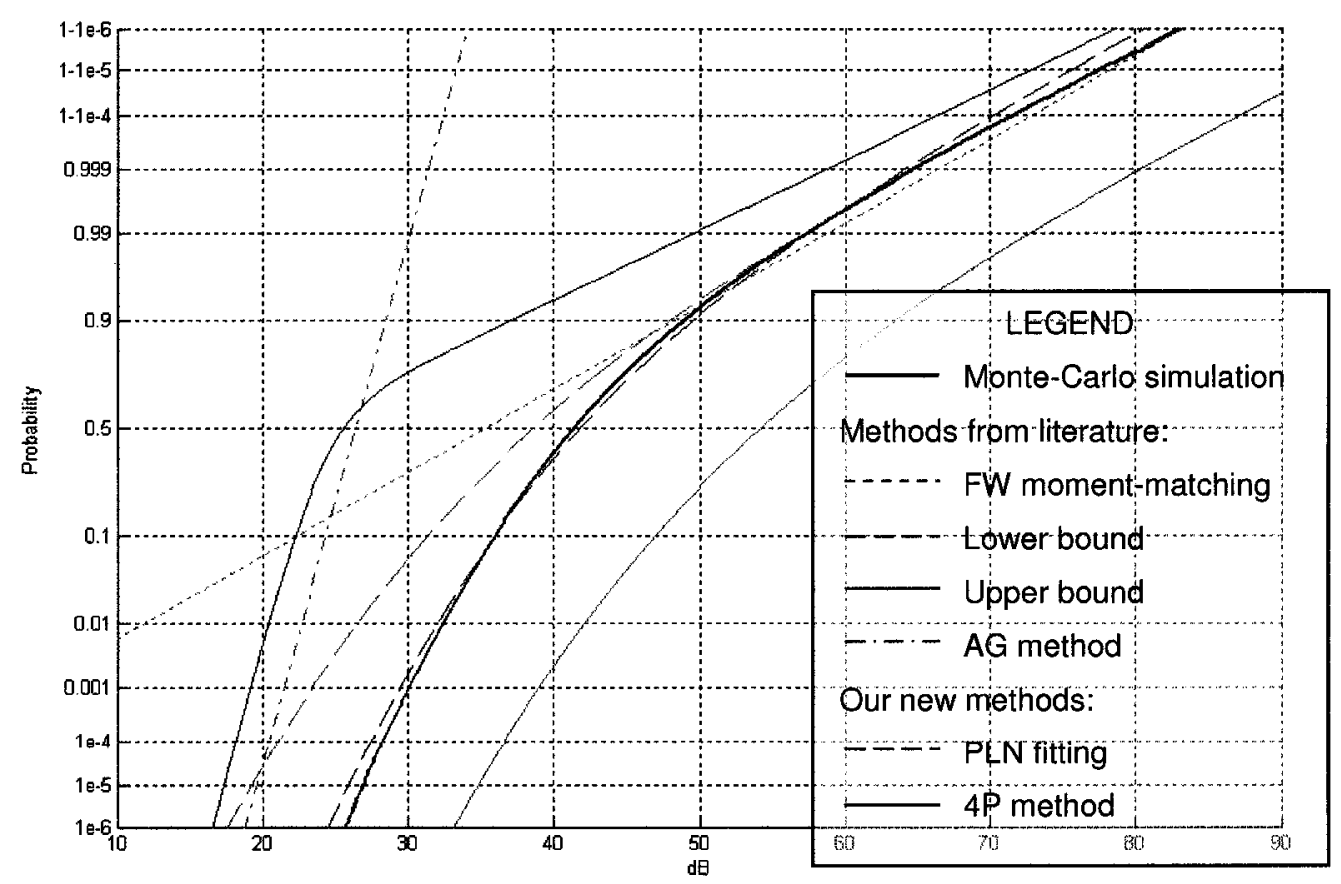

Figure 3-8 The cdf of the sum of 36 lognormals with different means and variances, and closed-form methods. 


\section{Chapter 4 Statistical Analysis of Total Interference Power}

In cellular communications, the purpose of the mathematical problem of the SLN is to find the distribution of the total interference power $I$ experienced by the DMU. We would like to do this without simulations, preferably with as little numerical methods as possible, ideally in simple closed form. We will use two different methods to find this distribution: one is the well-known FW moment-matching method [26], which is the simplest one and is entirely closed form. We found that no other closed-from method really existed that we could use with good results, which is the reason for our work in chapter 3. The second method we use is the PLN approximation, where we apply the method developed in Section 3.3.1 to the cellular interference problem.

In this chapter, we first show that the interference $I_{j}$ coming from one cell can be well-modelled by a Bernoulli Lognormal (BLN) RV. The sum of BLN is a more difficult problem, so we need to modify our SLN methods slightly. For both methods, we devise an exact method that is a bit long, as well as handy approximations that turn out to be good and can be essentially calculated with a scientific calculator and the Tables provided in this chapter. In order to give credibility to our results we verify our solutions by Monte-Carlo simulations, which is sometimes lacking in literature [1], [3]. We find that our methods tend to predict the distribution well in most cases, but not always, clearly indicating that more work needs to be done in the SLN problem. We believe that a better method can be found by pursuing the direction of Sections 3.2.2 and 3.3, which looks promising in that both tails could be fitted using closed-form equations.

\subsection{Simulation Algorithm}

Figures 4-2 and 4-3 give a flowchart of the algorithm that simulates interference in a cellular system according to the model given above. We used this algorithm to create our simulated 
curves. It is necessary to generate MU positions (both DMU and IMU) according to a spatially uniform distribution within a hexagonal cell of unit side. Also the positions of all the IFS's are needed. We can represent these as complex numbers, and then distances can be calculated as magnitudes of differences of positions. Figure 4-1 gives an example of a layout with normalised distances and mapped onto a complex plane.

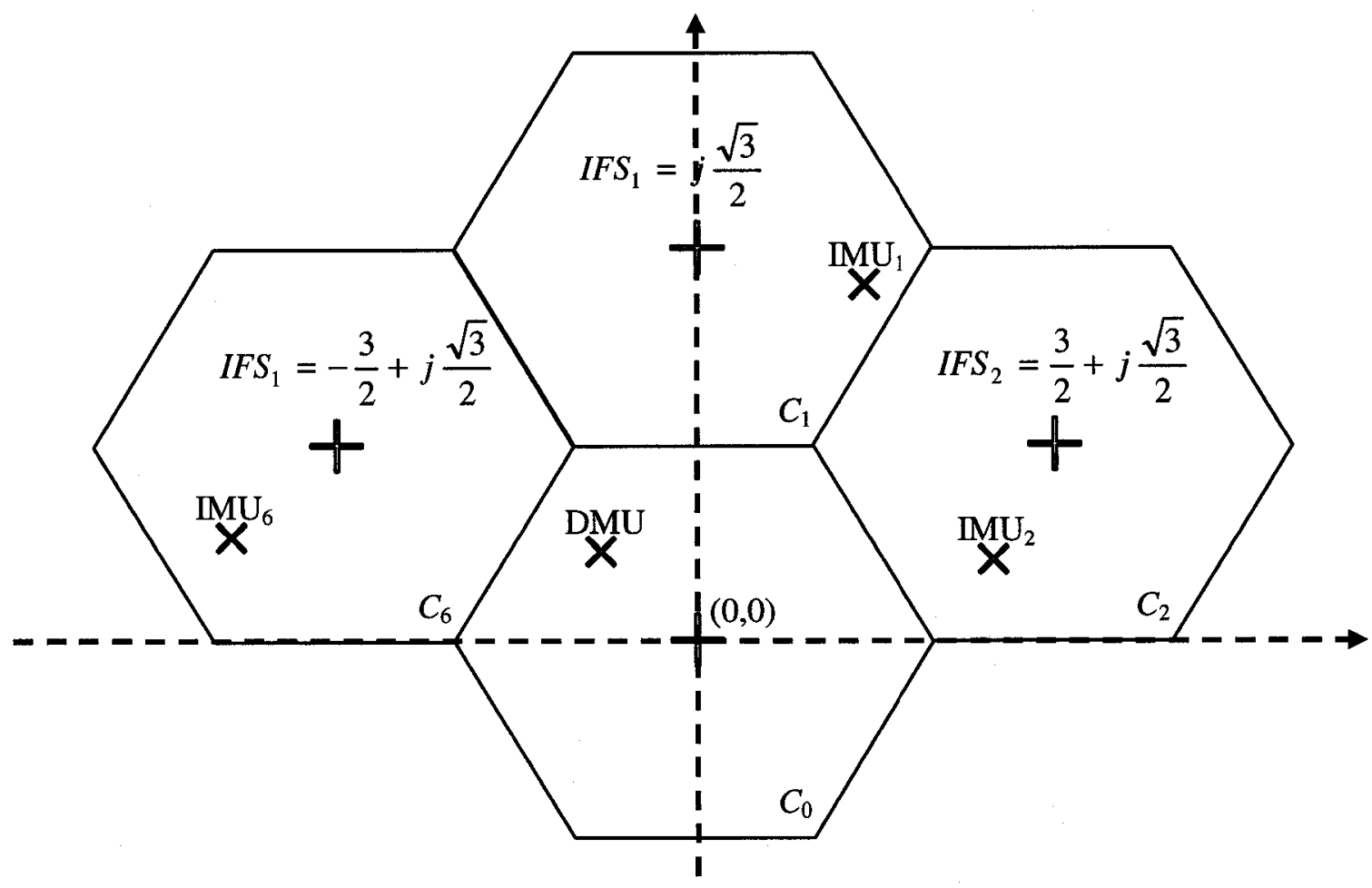

Figure 4-1 Normalised MU and FS positions in a complex plane. 


INITIALISATION:
1. $t \leftarrow 0$ Counts the number of generated
realizations of $I$, up to TRIALS.
2. Divide the range of values that $I$ can take into
bins, in a logarithmic fashion. The number of
realizations that fall into each bin is counted
by $H(b) \leftarrow 0 \forall b$.
3. Simulation parameters: $\beta, K, p, \sigma, \sigma_{e}, k_{P C}, k_{D L}$,
$N,\left\{I F S_{i}\right\}_{i=1}^{N}$ positions of the interfering FS's.

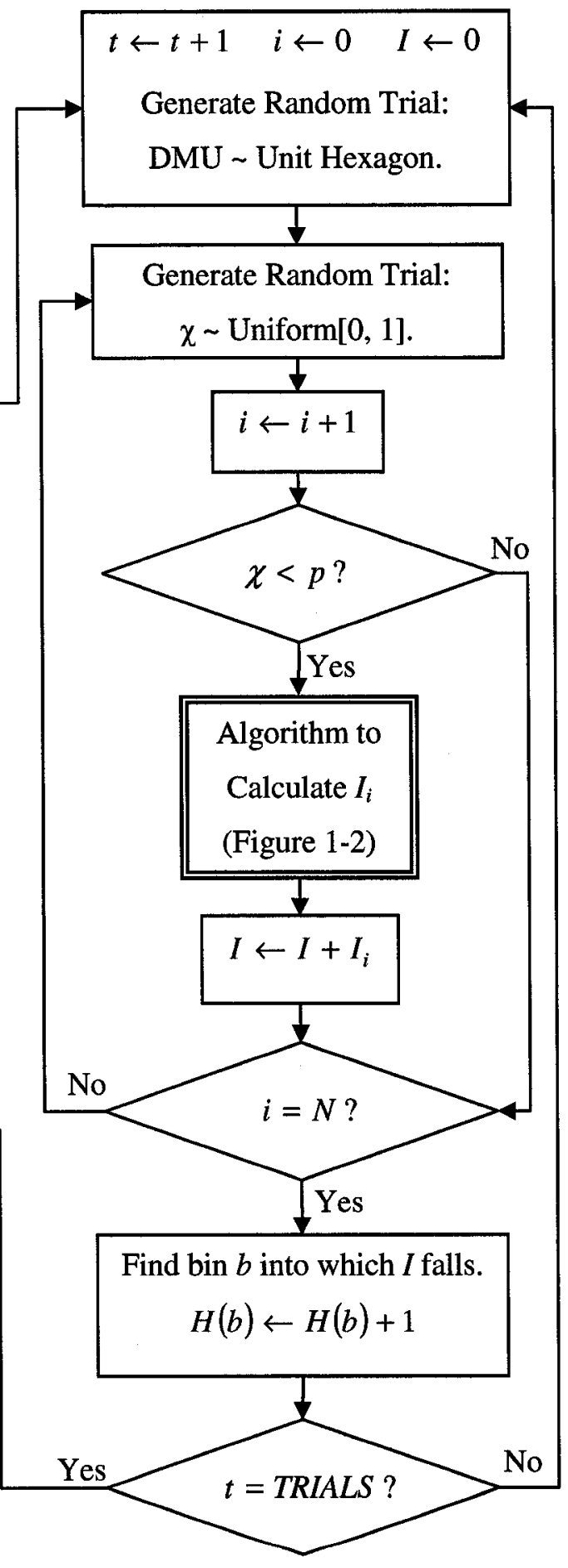

Figure 4-2 Algorithm for simulating interference in cellular systems. 


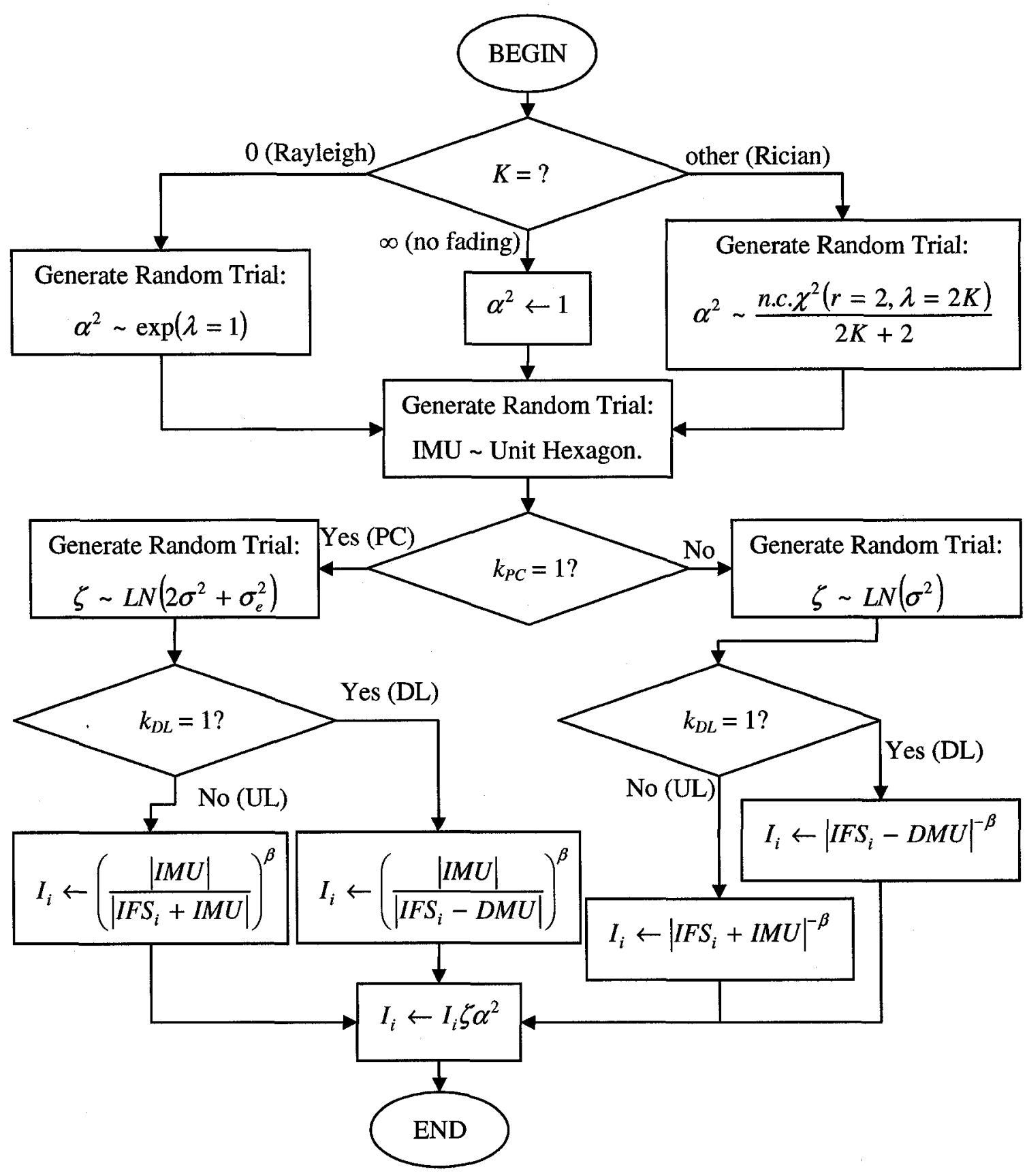

Figure 4-3 Algorithm to calculate $I_{\text {: }}$. 


\subsection{Distribution of the Interference Power from One Interferer}

We first want to see what is the (approximate) distribution of the individual terms $I_{j}$.

\subsubsection{Separating the Pathloss from all Other Factors}

An important insight is that the pathloss is independent of shadowing, fading, activity, and in the $\mathrm{DL}$, of transmit power due to $\mathrm{PC}$. We can rewrite (1.1) as:

$$
Q_{i}=\zeta_{i} \chi_{i} \alpha_{i}^{2} P_{i} \Rightarrow I=\sum_{i=1}^{N} R_{i} Q_{i}
$$

The case of UL - PC requires a slight modification, as the transmit power and pathloss both depend simultaneously on the IMU position. Hence we can essentially separate the geometry of the problem from all other considerations, and can tabulate coefficients that are a function only of the location of the cell and the pathloss exponent. The coefficients are found by numerical integration, and here is the beauty of the method, need not be recalculated ever again, and do not change with other system parameters. This is the approach proposed in [3]. We found in literature [1], [6], [7] that what is often attempted is to integrate the pathloss distribution together with shadowing, fading, etc., but we feel this is "reinventing the wheel" at each time, if we only want the moments of the resulting RV. It is much more convenient to be able to treat the geometry of the problem separately, tabulate it, and then work on the problem at hand without going into any layout considerations. This is a core idea of this chapter, and leads naturally to our goal of obtaining simple, closed-form solutions to the interference distribution.

This separation of geometry is also important because all other factors: shadowing, fading, transmit power and activity are i.i.d. across the cells, whereas the pathlosses are both statistically different, and also, in DL, correlated. For all these reasons, we believe that the separation is a judicious one. 


\subsubsection{Statistical Moments of the Inverse Pathloss}

The moments of the individual inverse pathlosses need to be calculated by numerical integration, though analytical approximations exist [3], [5], [10]:

$$
\kappa_{i}(n \beta)=E\left[R_{i}^{n}\right]=\frac{2}{3 \sqrt{3}} \iiint_{(x, y) \in C_{0}}{\sqrt{\left(x-x_{i}\right)^{2}+\left(y-y_{i}\right)^{2}}}^{-n \beta} d x d y
$$

Here $C_{0}$ is the cell under consideration, enclosed by a unit hexagon centered at the origin, and ( $x_{i}$, $y_{i}$ ) are the coordinates of IFS $i$. Figure 4-1 illustrates a possible layout. We tabulate these values for all cells in a traditional three-tier cellular layout (Figure 4-4). We see in the Figure that there are five different classes of cells, where cells in the same class are located in the same manner with respect to the cell under consideration $C_{0}$. Thus we need to calculate $\kappa$ coefficients for only five different cells (Tables 4-1 and 4-2).

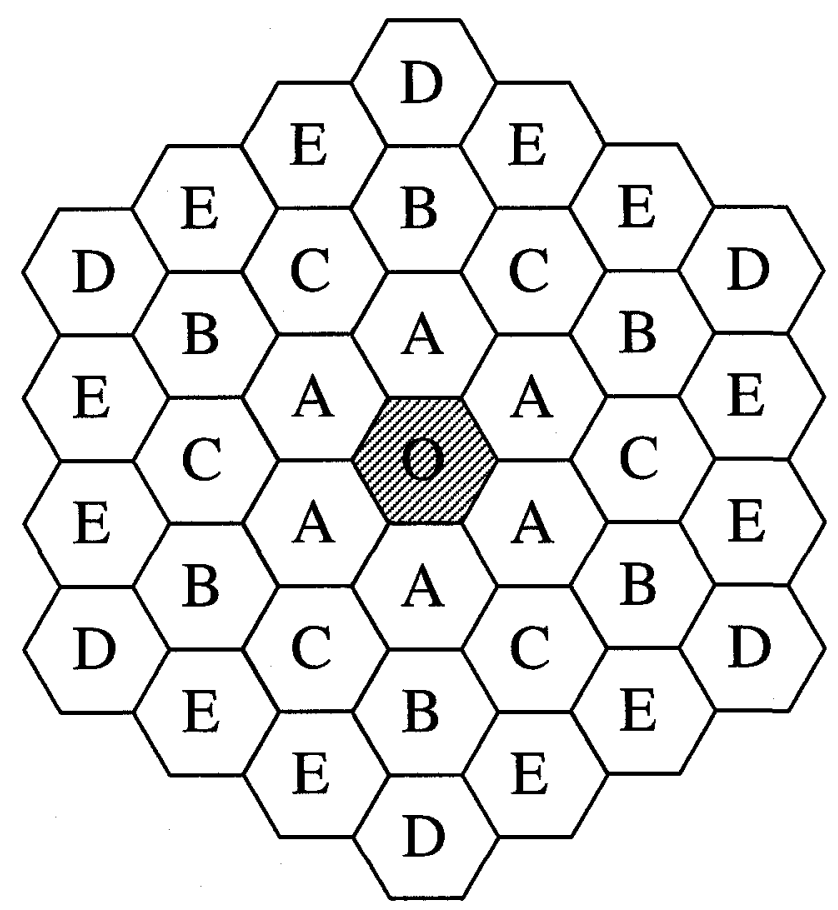

Figure 4-4 Three tiers of cells with five different cell positions (classes A-E). 


\begin{tabular}{cccccc}
\hline \hline $\boldsymbol{\beta}$ & Class A & Class B & Class C & Class D & Class E \\
\hline $\mathbf{3}$ & 0.2798 & 0.02609 & 0.04130 & 0.007384 & 0.01088 \\
$\mathbf{3 . 2}$ & 0.2645 & 0.02058 & 0.03365 & 0.005336 & 0.008072 \\
$\mathbf{3 . 4}$ & 0.2508 & 0.01624 & 0.02745 & 0.003858 & 0.005993 \\
$\mathbf{3 . 5}$ & 0.2446 & 0.01443 & 0.02480 & 0.003281 & 0.005165 \\
$\mathbf{3 . 6}$ & 0.2387 & 0.01283 & 0.02242 & 0.002790 & 0.004452 \\
$\mathbf{3 . 8}$ & 0.2279 & 0.01014 & 0.01832 & 0.002018 & 0.003308 \\
$\mathbf{4}$ & 0.2184 & 0.608023 & 0.01499 & $0.001461 /$ & 0.002459 \\
$\mathbf{4 . 2}$ & 0.2099 & 0.006351 & 0.01227 & 0.001057 & 0.001829 \\
$\mathbf{4 . 4}$ & 0.2024 & 0.005031 & 0.01006 & 0.0067655 & 0.001361 \\
$\mathbf{4 . 5}$ & 0.1990 & 0.004479 & 0.009108 & 0.0006515 & 0.001174 \\
$\mathbf{4 . 6}$ & 0.1958 & 0.003988 & 0.008250 & 0.0005545 & 0.001013 \\
$\mathbf{4 . 8}$ & 0.1900 & 0.003164 & 0.006774 & 0.0004018 & 0.0007540 \\
$\mathbf{5}$ & 0.1849 & 0.002512 & 0.005567 & 0.0002912 & 0.0005617 \\
\hline \hline
\end{tabular}

Table 4-1 $\kappa_{i}(\beta)$ function tabulated for all cell classes and pathloss exponents.

\begin{tabular}{cccccc}
\hline \hline $\boldsymbol{\beta}$ & Class A & Class B & Class C & Class D & Class E \\
\hline $\mathbf{3}$ & 0.1680 & 0.0007999 & 0.002113 & 0.00005849 & 0.0001296 \\
$\mathbf{3 . 2}$ & 0.1645 & 0.0005084 & 0.001443 & 0.00003085 & 0.00007224 \\
$\mathbf{3 . 4}$ & 0.1624 & 0.0003240 & 0.0009882 & 0.00001629 & 0.00004035 \\
$\mathbf{3 . 5}$ & 0.1618 & 0.0002590 & 0.0008187 & 0.00001184 & 0.00003017 \\
$\mathbf{3 . 6}$ & 0.1616 & 0.0002070 & 0.0006788 & 0.000008608 & 0.00002257 \\
$\mathbf{3 . 8}$ & 0.1620 & $0.0001(325$ & 0.0004676 & 0.000004555 & 0.00001264 \\
$\mathbf{4}$ & 0.1634 & 0.00008598 & 0.0003230 & 0.000002413 & 0.000007090 \\
$\mathbf{4 . 2}$ & 0.1659 & 0.00005473 & 0.0002237 & 0.000001280 & 0.000003982 \\
$\mathbf{4 . 4}$ & 0.1693 & 0.00003529 & 0.0001553 & 0.0000006796 & 0.000002240 \\
$\mathbf{4 . 5}$ & 0.1714 & 0.00002836 & 0.0001295 & 0.0000004954 & 0.000001681 \\
$\mathbf{4 . 6}$ & 0.1737 & 0.00002280 & 0.0001081 & 0.0000003612 & 0.000001262 \\
$\mathbf{4 . 8}$ & 0.1790 & 0.00001476 & 0.00007536 & 0.0000001922 & 0.0000007116 \\
$\mathbf{5}$ & 0.1853 & 0.000009576 & 0.00005267 & 0.0000001024 & 0.0000004019 \\
\hline \hline
\end{tabular}

Table 4-2 $\kappa_{i}(2 \beta)$ function tabulated for all cell classes and pathloss exponents. 


\subsubsection{Statistical Moments of all Other Factors}

Since the components of $Q_{i}$ are independent, and all $Q_{i}$ are i.i.d., we may define the $B$-coefficients as follows:

$$
B_{n}=E\left[Q_{i}^{n}\right]=E\left[\zeta_{i}^{n}\right] E\left[\alpha_{i}^{2 n}\right] E\left[\chi_{i}^{n}\right] E\left[P_{i}^{n}\right], \forall i
$$

If there is no PC: $P_{i}=1, \forall i$.

With PC, the power transmitted will be proportional to the pathloss between the IMU $i$ and its FS. The position of the IMU has uniform distribution over its cell area $C_{i}$. Now the cell area is a regular hexagon of unit side, which it is conventional to approximate by a circle of radius $\rho \leq 1$. We define the mean pathloss between an IMU $i$ and its own IFS to be $L_{i}$ :

$$
\begin{gathered}
L_{i} \sim f_{L}(x)=\frac{2}{\beta \rho^{2}} x^{\frac{2}{\beta}-1}, x \in\left[0, \rho^{\beta}\right], \\
E\left[L_{i}^{n}\right]=\frac{2}{2+n \beta} \rho^{n \beta} .
\end{gathered}
$$

In [1], [3], $\rho$ is assumed 1. We found, through numerical integration, that $\rho=0.915$ gives a good approximation for the first two moments and use this value in our analysis. A value of $3^{\frac{3}{4}} / \sqrt{2 \pi} \cong 0.9094$ is used in [5] and [10].

The transmit power will also be affected by LN shadowing, for which the PC mechanism will attempt to compensate. We model this by a $\mathrm{LN}$ variable with statistics $\left(0, \sigma^{2}+\sigma_{e}^{2}\right)$, as done in [1]. Finally, with PC, the moments of the transmit power are:

$$
E\left[P_{i}^{n}\right]=\frac{2}{2+n \beta} \rho^{n \beta} e^{\frac{n^{2}}{2}\left(\sigma^{2}+\sigma_{e}^{2}\right)}
$$

Consequently: 


$$
B_{n}=p E\left[\alpha_{i}^{2 n}\right] e^{\frac{n^{2}}{2} \sigma^{2}}\left[\frac{2}{2+n \beta} \rho^{n \beta} e^{\frac{n^{2}}{2}\left(\sigma^{2}+\sigma_{e}^{2}\right)}\right]^{k_{P C}}
$$

We normalise the fading power such that the power mean $E\left[\alpha_{i}^{2}\right]=1$. Then the second moment of the power is known [59]:

$$
E\left[\alpha_{i}^{4}\right]=2-\left(1+K^{-1}\right)^{-2}
$$

Note that when there is no fading: $K=\infty, E\left[\alpha_{i}^{4}\right]=1$. For Rayleigh fading, $K=0 \Rightarrow E\left[\alpha_{i}^{4}\right]=2$. We then obtain:

$$
\begin{gathered}
B_{1}=p e^{\frac{1}{2} \sigma^{2}}\left[\frac{2}{2+\beta} \rho^{\beta} e^{\frac{1}{2}\left(\sigma^{2}+\sigma_{e}^{2}\right)}\right]^{k_{P C}}, \\
B_{2}=p\left(2-\left(1+K^{-1}\right)^{-2}\right) e^{2 \sigma^{2}}\left[\frac{1}{1+\beta} \rho^{2 \beta} e^{2 \sigma^{2}+2 \sigma_{e}^{2}}\right]^{k_{P C}}
\end{gathered}
$$

We can write, for all cases except UL - PC, that the pathloss is independent of all other factors, which leads to:

$$
E\left[I_{i}^{n}\right]=E\left[R_{i}^{n} Q_{i}^{n}\right]=E\left[R_{i}^{n}\right] E\left[Q_{i}^{n}\right]=\kappa_{i}(n \beta) B_{n} \quad\left[k_{D L}, k_{P C}\right] \neq[0,1]
$$

However in the case of UL-PC, $R_{i}$ and $Q_{i}$ are not independent, and a modified method needs to be developed. 


\begin{tabular}{clcccc}
\hline \hline $\boldsymbol{\beta}$ & Class A & Class B & Class C & Class D & Class E \\
\hline $\mathbf{3}$ & 0.2799 & 0.02609 & 0.04130 & 0.007384 & 0.01088 \\
$\mathbf{3 . 2}$ & 0.2645 & 0.02058 & 0.03365 & 0.005336 & 0.008072 \\
$\mathbf{3 . 4}$ & 0.2508 & 0.01624 & 0.02745 & 0.003858 & 0.005993 \\
$\mathbf{3 . 5}$ & 0.2446 & 0.01443 & 0.02480 & 0.003281 & 0.005165 \\
$\mathbf{3 . 6}$ & 0.2387 & 0.01283 & 0.02242 & 0.002790 & 0.004452 \\
$\mathbf{3 . 8}$ & 0.2279 & 0.01014 & 0.01832 & 0.002018 & 0.003308 \\
$\mathbf{4}$ & 0.2184 & 0.008023 & 0.01499 & $0.001461 /$ & 0.002459 \\
4.2 & 0.2099 & 0.006351 & 0.01227 & 0.001057 & 0.001829 \\
$\mathbf{4 . 4}$ & 0.2024 & 0.005031 & 0.01006 & 0.0007655 & 0.001361 \\
4.5 & 0.1990 & 0.004479 & 0.009108 & 0.0006515 & 0.001174 \\
4.6 & 0.1958 & 0.003988 & 0.008250 & 0.0005545 & 0.001013 \\
4.8 & 0.1900 & 0.003164 & 0.006774 & 0.0004018 & 0.000754 \\
$\mathbf{5}$ & 0.1849 & 0.002512 & 0.005567 & 0.0002912 & 0.0005617 \\
\hline \hline
\end{tabular}

Table 4-3 $\widetilde{\kappa}_{i}(\beta)$ function tabulated for all cell classes and pathloss exponents.

\begin{tabular}{cccccc}
\hline \hline $\boldsymbol{\beta}$ & Class A & Class B & Class C & Class D & Class E \\
\hline $\mathbf{3}$ & 0.1680 & 0.0007999 & 0.002113 & 0.00005849 & 0.0001296 \\
$\mathbf{3 . 2}$ & 0.1645 & 0.0005084 & 0.001443 & 0.00003085 & 0.00007224 \\
$\mathbf{3 . 4}$ & 0.1624 & 0.0003240 & 0.0009882 & 0.00001629 & 0.00004035 \\
$\mathbf{3 . 5}$ & 0.1618 & 0.0002589 & 0.0008187 & 0.00001184 & 0.00003017 \\
$\mathbf{3 . 6}$ & 0.1616 & 0.0002070 & 0.0006788 & 0.000008608 & 0.00002257 \\
$\mathbf{3 . 8}$ & 0.1620 & 0.0001325 & 0.0004676 & 0.000004555 & 0.00001264 \\
$\mathbf{4}$ & 0.1634 & 0.00008508 & 0.0003230 & 0.000002413 & 0.00000709 \\
$\mathbf{4 . 2}$ & 0.1659 & 0.00005473 & 0.0002237 & $0.00000 / 28$ & 0.000003982 \\
$\mathbf{4 . 4}$ & 0.1693 & 0.00003529 & 0.0001553 & 0.0000006796 & 0.00000224 \\
$\mathbf{4 . 5}$ & 0.1714 & 0.00002836 & 0.0001295 & 0.0000004954 & 0.000001681 \\
$\mathbf{4 . 6}$ & 0.1737 & 0.00002280 & 0.0001081 & 0.0000003612 & 0.000001262 \\
$\mathbf{4 . 8}$ & 0.1790 & 0.00001476 & 0.00007536 & 0.0000001922 & 0.0000007116 \\
$\mathbf{5}$ & 0.1853 & 0.000009576 & 0.00005267 & 0.0000001024 & 0.0000004019 \\
\hline \hline
\end{tabular}

Table 4-4 $\widetilde{\kappa}_{i}(2 \beta)$ function tabulated for all cell classes and pathloss exponents. 


\subsubsection{The Special Case of Uplink with Power Control}

In the UL - PC case, the pathloss between the IMU and the FS of cell $C_{0}$ and the pathloss between the IMU and its own FS are two correlated RV's. We modify our method as follows:

$$
\tilde{\kappa}_{i}(n \beta)=E\left[R_{i}^{n} L_{i}^{n}\right]=\frac{2}{3 \sqrt{3}} \iiint_{(x, y) \in C_{0}} \sqrt{\left(\left(x-x_{i}\right)^{2}+\left(y-y_{i}\right)^{2}\right)\left(x_{i}^{2}+y_{i}^{2}\right)^{-n \beta}} d x d y .
$$

Here the integral is performed in the same manner as (4.2). These values are tabulated in Tables 4-3 and 4-4. We also define:

$$
\begin{gathered}
\tilde{B}_{n}=E\left[\frac{Q_{i}^{n}}{L_{i}^{n}}\right]=E\left[\zeta_{i}^{n}\right] E\left[\alpha_{i}^{2 n}\right] E\left[\chi_{i}^{n}\right] E\left[\frac{P_{i}^{n}}{L_{i}^{n}}\right], \forall i \\
\tilde{B}_{n}=p E\left[\alpha_{i}^{2 n}\right] e^{\frac{n^{2}}{2}\left(2 \sigma^{2}+\sigma_{e}^{2}\right)} . \\
\tilde{B}_{1}=p e^{\sigma^{2}+\frac{1}{2} \sigma_{e}^{2}} \\
\tilde{B}_{2}=p\left(2-\left(1+K^{-1}\right)^{-2}\right) e^{4 \sigma^{2}+2 \sigma_{e}^{2}}
\end{gathered}
$$

Then the moments can be expressed as:

$$
E\left[I_{i}^{n}\right]=E\left[R_{i}^{n} Q_{i}^{n}\right]=E\left[R_{i}^{n} L_{i}^{n}\right] E\left[\frac{Q_{i}^{n}}{L_{i}^{n}}\right]=\widetilde{\kappa}_{i}(n \beta) \tilde{B}_{n} \quad\left[k_{D L}, k_{P C}\right]=[0,1]
$$

\subsubsection{Bernoulli Lognormal Approximation to Single Interferer Power Distribution}

The signal power coming from a single interferer, $I_{j}$, can be modeled approximately by a BLN $\mathrm{RV}$, with $c d f$ : 


$$
F_{i}(x) \cong \Phi\left(\frac{\ln x-a_{i}}{b_{i}}\right) p+(1-p), x>0
$$

\subsubsection{Support in Literature}

We model the interference from one user as a product of a LN shadowing component, a fading (Rayleigh, Rician or none) component, a pathloss component that assumes some spatial distribution of the MU. Also, if there is PC, the transmit power is not constant, but follows a distribution that is LN (compensating for shadowing and also because of LN PC error), multiplied by a quantity proportional to the pathloss (so as to compensate for it) between a randomly located IMU and its FS. The product of all these is approximated as a LN RV. Furthermore, user activity is modelled as Bernoulli, thus the interference power from a given interferer is modelled as BLN. This is the same approximation as in [1].

\subsubsection{Theoretical Basis}

It is not accidental that the distribution of the product of many RV's approaches a $\mathrm{LN}$ distribution, particularly if some of the terms are already LN, with high variance. First of all, the product of LN RV's is also LN. Secondly, by virtue of the Central Limit Theorem for products, the (properly normalised) product of many RV's with square-integrable $p d f$ 's will approach a LN RV in distribution [60]. The Bernoulli RV does not satisfy the condition and thus cannot be absorbed in the LN approximation. Indeed, multiplying any number of Bernoulli RV's can only result in another Bernoulli RV. The Central Limit Theorem for products works quite well in our case: though only a few RV's are multiplied, there are already among them LN RV's with high variance: the shadowing (6-12 dB) and, if there is PC, another shadowing factor due to the power compensation. Thus we expect the approximation to be good (and get better, with higher shadowing). 


\subsubsection{Fitting the Distribution by Moment-Matching}

The moments of $I_{j}$ can be matched with those of the approximating distribution $F_{i}(x)$ :

$$
E\left[I_{i}^{n}\right]=p \cdot e^{n a_{i}+\frac{n^{2}}{2} b_{i}^{2}}
$$

Inverting (4.19), we obtain:

$$
\begin{aligned}
& a_{i}=2 \ln E\left[I_{i}\right]-\frac{1}{2} \ln E\left[I_{i}^{2}\right]-\frac{3}{2} \ln p \\
& =\left\{\begin{array}{c}
2 \ln \kappa_{i}(\beta)-\frac{1}{2} \ln \kappa_{i}(2 \beta)-\frac{3}{2} \ln p-\frac{1}{2} \ln \left(2-\left(1+K^{-1}\right)^{-2}\right) \\
+k_{P C}\left[\beta \ln \rho-2 \ln \left(1+\frac{1}{2} \beta\right)+\frac{1}{2} \ln (1+\beta)\right] \quad\left[k_{D L}, k_{P C}\right] \neq[0,1] \\
2 \ln \widetilde{\kappa}_{i}(\beta)-\frac{1}{2} \ln \tilde{\kappa}_{i}(2 \beta)-\frac{3}{2} \ln p-\frac{1}{2} \ln \left(2-\left(1+K^{-1}\right)^{-2}\right) \quad\left[k_{D L}, k_{P C}\right]=[0,1],
\end{array}\right. \\
& b_{i}^{2}=-2 \ln E\left[I_{i}\right]+\ln E\left[I_{i}^{2}\right]+\ln p \\
& =\left\{\begin{array}{c}
\sigma^{2}-2 \ln \kappa_{i}(\beta)+\ln \kappa_{i}(2 \beta)+\ln p+\ln \left(2-\left(1+K^{-1}\right)^{-2}\right) \\
+k_{P C}\left[\sigma^{2}+\sigma_{e}^{2}+2 \ln \left(1+\frac{1}{2} \beta\right)-\ln (1+\beta)\right] \quad\left[k_{D L}, k_{P C}\right] \neq[0,1] \\
2 \sigma^{2}+\sigma_{e}^{2}-2 \ln \tilde{\kappa}_{i}(\beta)+\ln \tilde{\kappa}_{i}(2 \beta)+\ln p+\ln \left(2-\left(1+K^{-1}\right)^{-2}\right) \quad\left[k_{D L}, k_{P C}\right]=[0,1] .
\end{array}\right.
\end{aligned}
$$

\subsubsection{Validation through Simulation}

We simulate the distribution of the interference coming from one nearby interferer (class $\mathrm{A}$ ), as well as from a distant interferer (class D) and compare the results with the theoretical LN distributions with parameters given by (4.20), (4.21). Note that we set $p=1$, since the pdf of a RV multiplied by a Bernoulli RV is just the scaled original pdf with additional mass at zero, and there is no need to simulate this. Also, when there is no PC, both UL and DL interference are the same for one user, and thus the simulated results apply equally to both cases.

The simulations are done according to the parameters in Table $4-5$, while $\sigma_{e}=1 \mathrm{~dB} \approx 0.23$. The results are plotted on Figures 4-5 to 4-10. We observe an excellent match in the higher values of 
the distributions, which can be expected, since moment-matching should give a good match to the upper tail [43], but the lower tails tend to diverge in most cases. The lower tail is wellmatched only when there is no fading, and the interfering cell is far, meaning that the pathloss will not vary overmuch, and the power is essentially LN (Figure 4-8). The results are not encouraging in the lower tail. However, we will see in subsequent sections that although this affects the results of the problem of many interferers, the error in the lower tail does not necessarily accumulate, and may be in fact be quite small.

\begin{tabular}{|c|c|c|c|c|c|c|c|c|c|}
\hline Figure & & $4-5$ & & & 4-6 & & & $4-7$ & \\
\hline$\beta$ & & 3 & & & 4 & & & 3.5 & \\
\hline$\sigma[\mathrm{dB}]$ & 6 & 9 & 12 & 6 & 9 & 12 & 6 & 9 & 12 \\
\hline $\boldsymbol{K}$ & & 0 & & & $\infty$ & & & 10 & \\
\hline Cell & & & & & Class A & & & & \\
\hline Link & & UL/DL & & & DL & & & UL & \\
\hline $\mathbf{P C}$ & & No & & & Yes & & & Yes & \\
\hline$a_{i}$ & & -2.002 & & & -3.884 & & & -3.567 & \\
\hline $\boldsymbol{b}_{i}$ & 1.837 & 2.400 & 3.017 & 2.312 & 3.181 & 4.099 & 2.290 & 3.165 & 4.086 \\
\hline Figure & & $4-8$ & & & 4-9 & & & 4-10 & \\
\hline$\beta$ & & 3.5 & & & 3 & & & 4 & \\
\hline$\sigma[\mathrm{dB}]$ & 6 & 9 & 12 & 6 & 9 & 12 & 6 & 9 & 12 \\
\hline $\boldsymbol{K}$ & & $\infty$ & & & 10 & & & 0 & \\
\hline Cell & & & & & Class D & & & & \\
\hline Link & & UL/DL & & & $\mathrm{DL}$ & & & UL & \\
\hline PC & & No & & & Yes & & & Yes & \\
\hline$a_{i}$ & & -5.767 & & & -6.430 & & & -8.685 & \\
\hline$b_{i}$ & 1.416 & 2.095 & 2.780 & 2.069 & 3.009 & 3.967 & 2.218 & 3.113 & 4.046 \\
\hline
\end{tabular}

Table 4-5 Simulation parameters for single interferer power. 


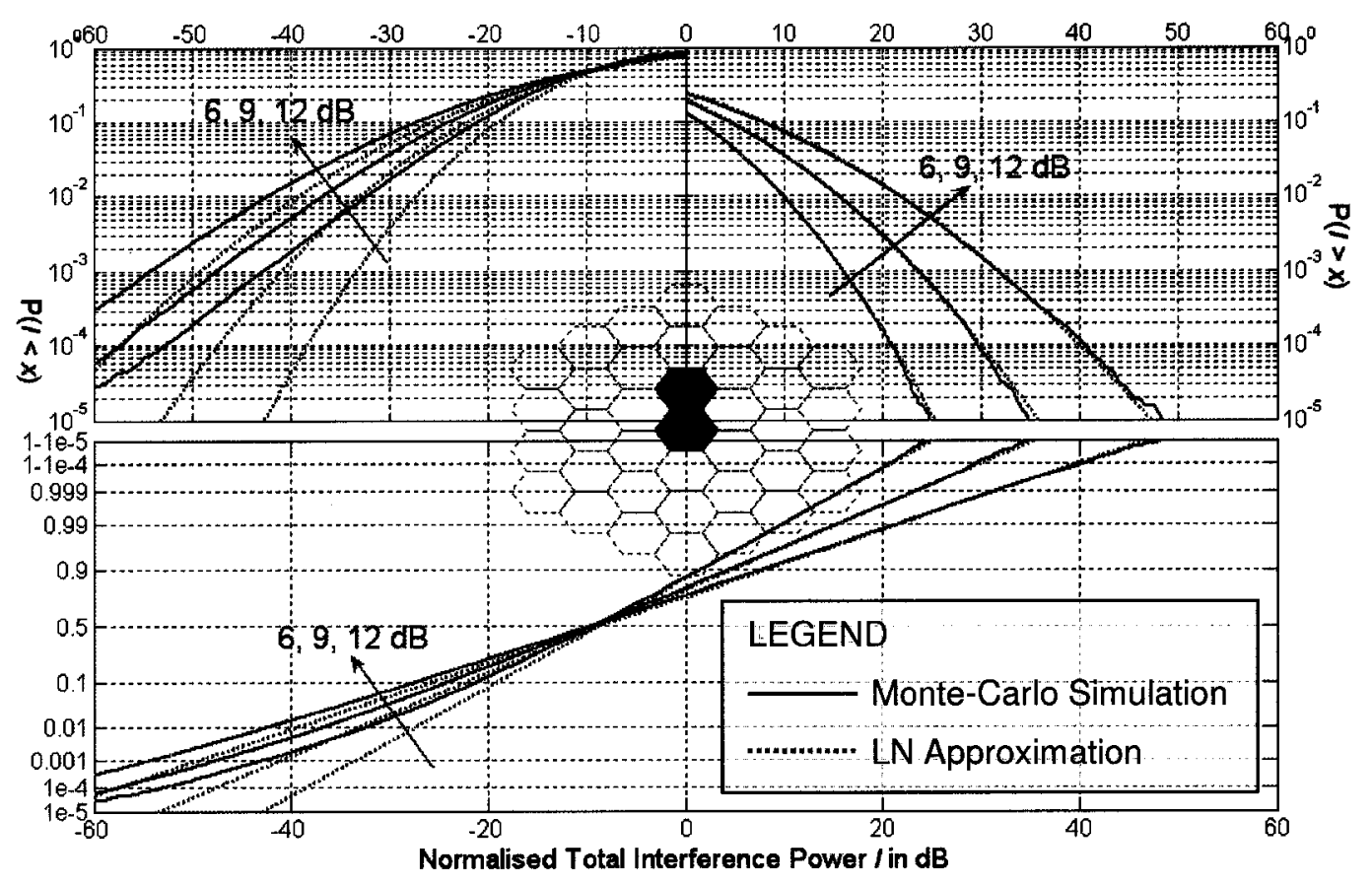

Figure 4-5 Single nearby interferer, Rayleigh fading, without power control.

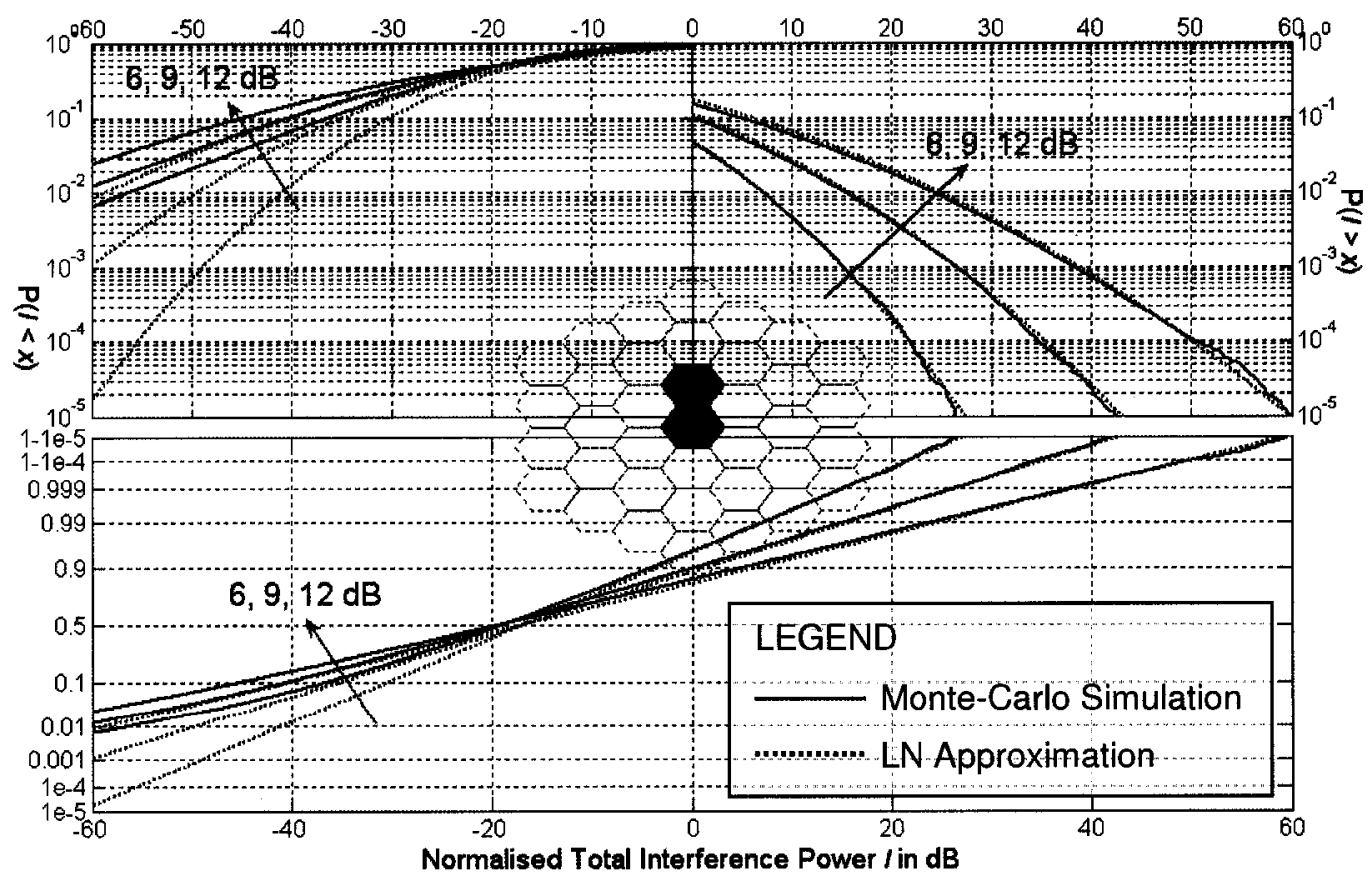

Figure 4-6 Single nearby interferer, no fading, downlink with power control. 


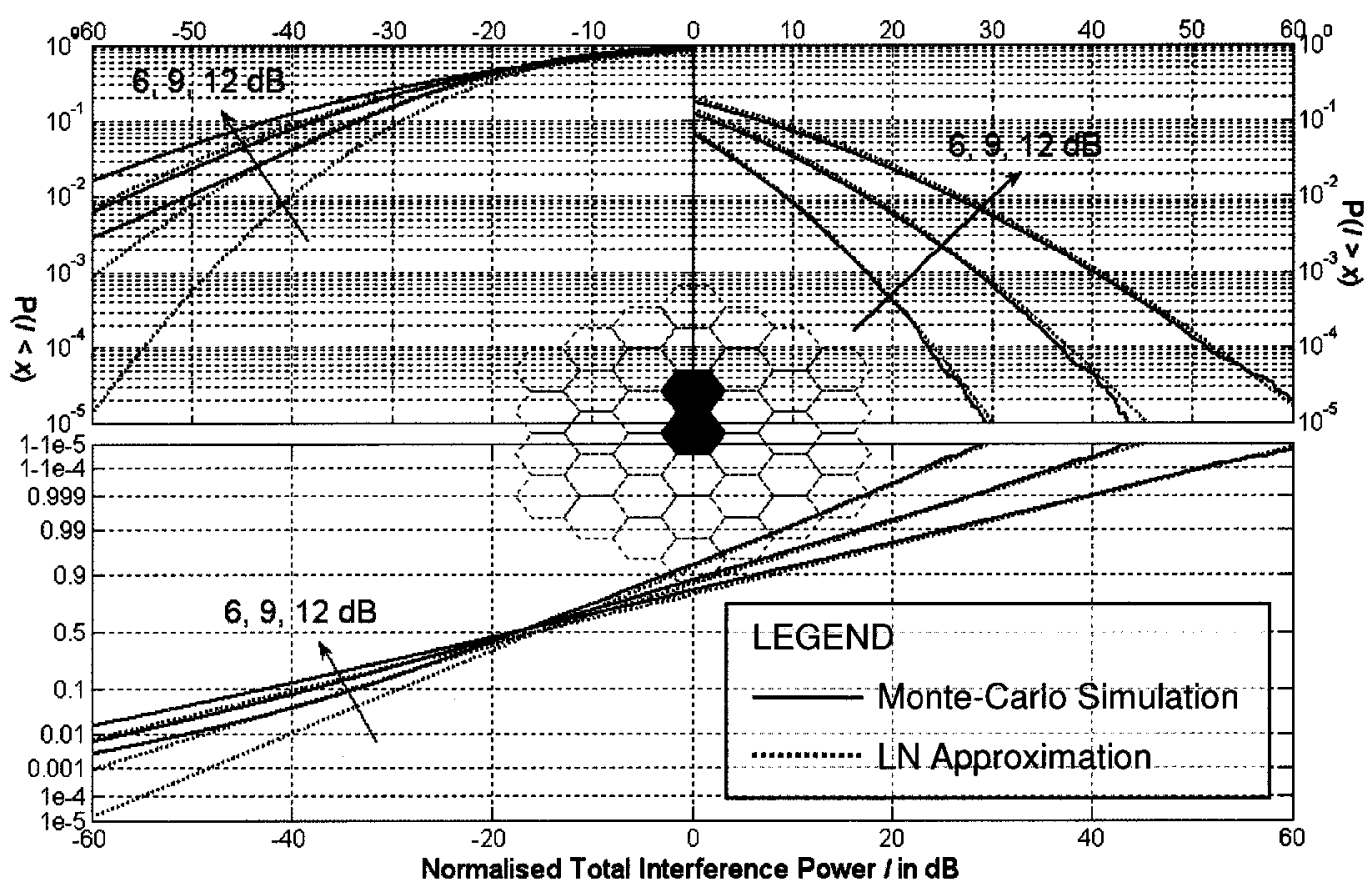

Figure 4-7 Single nearby interferer, Rician fading, uplink with power control.

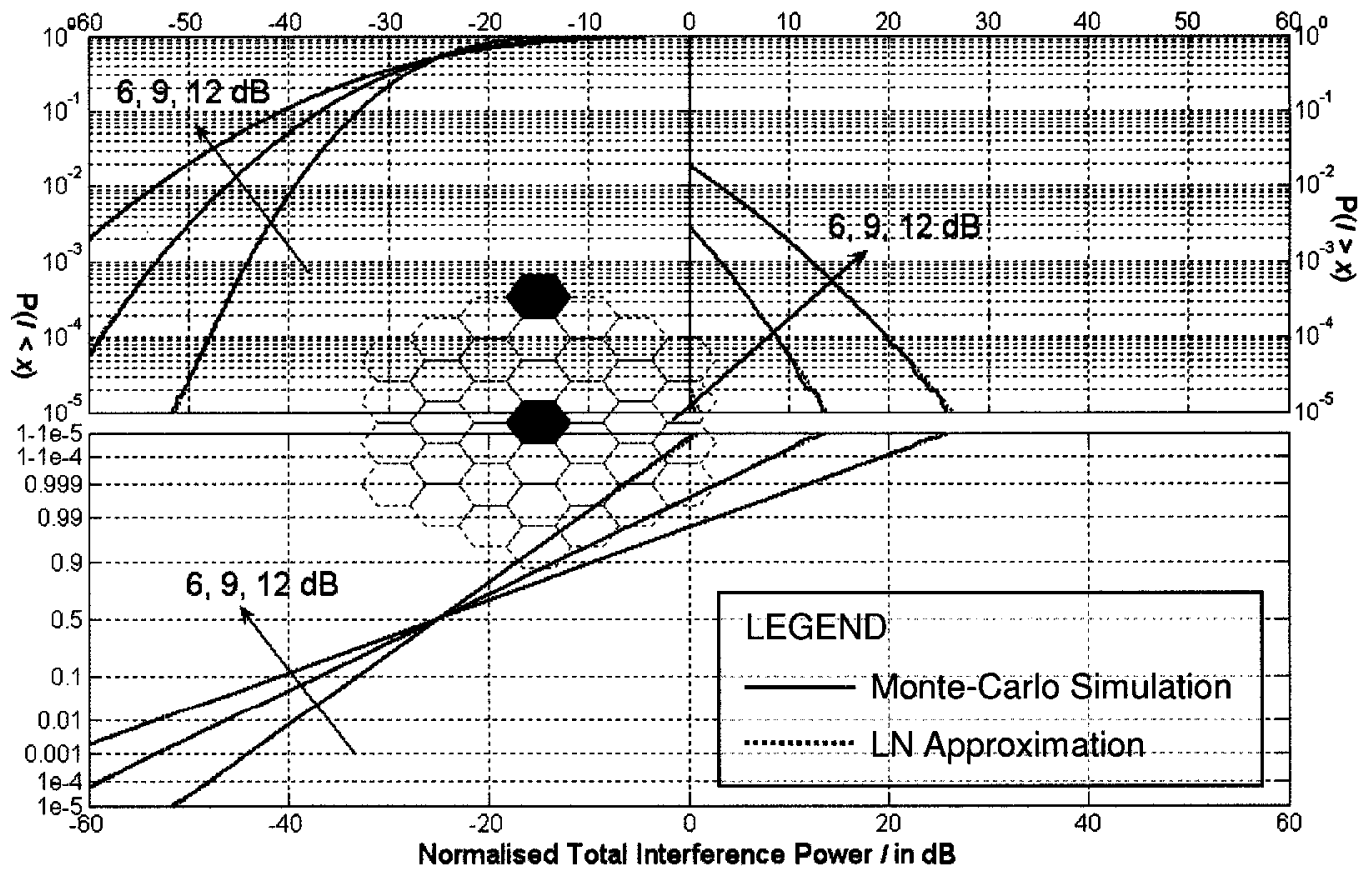

Figure 4-8 Single distant interferer, no fading, without power control. 


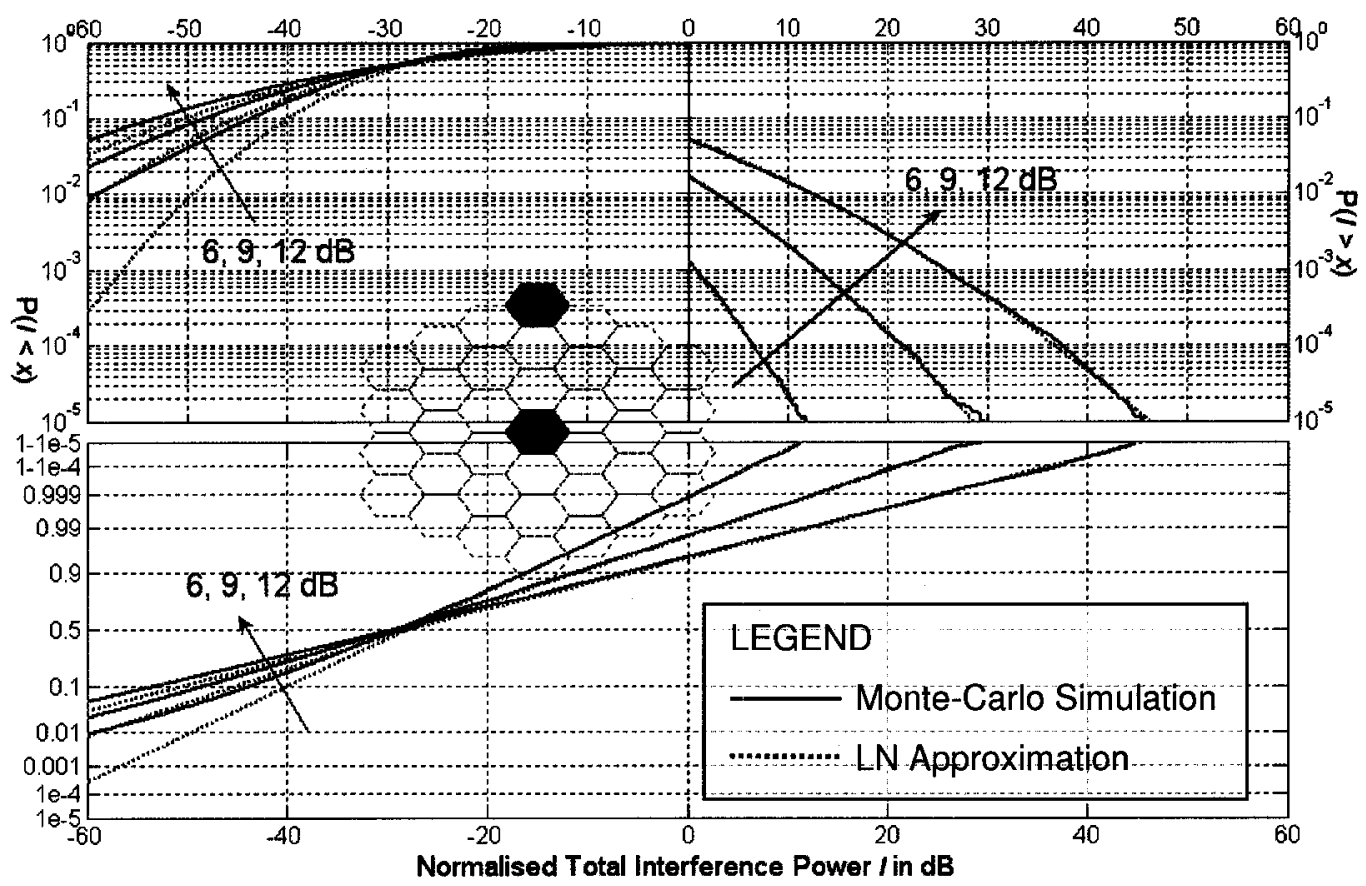

Figure 4-9 Single distant interferer, Rician fading, downlink with power control.

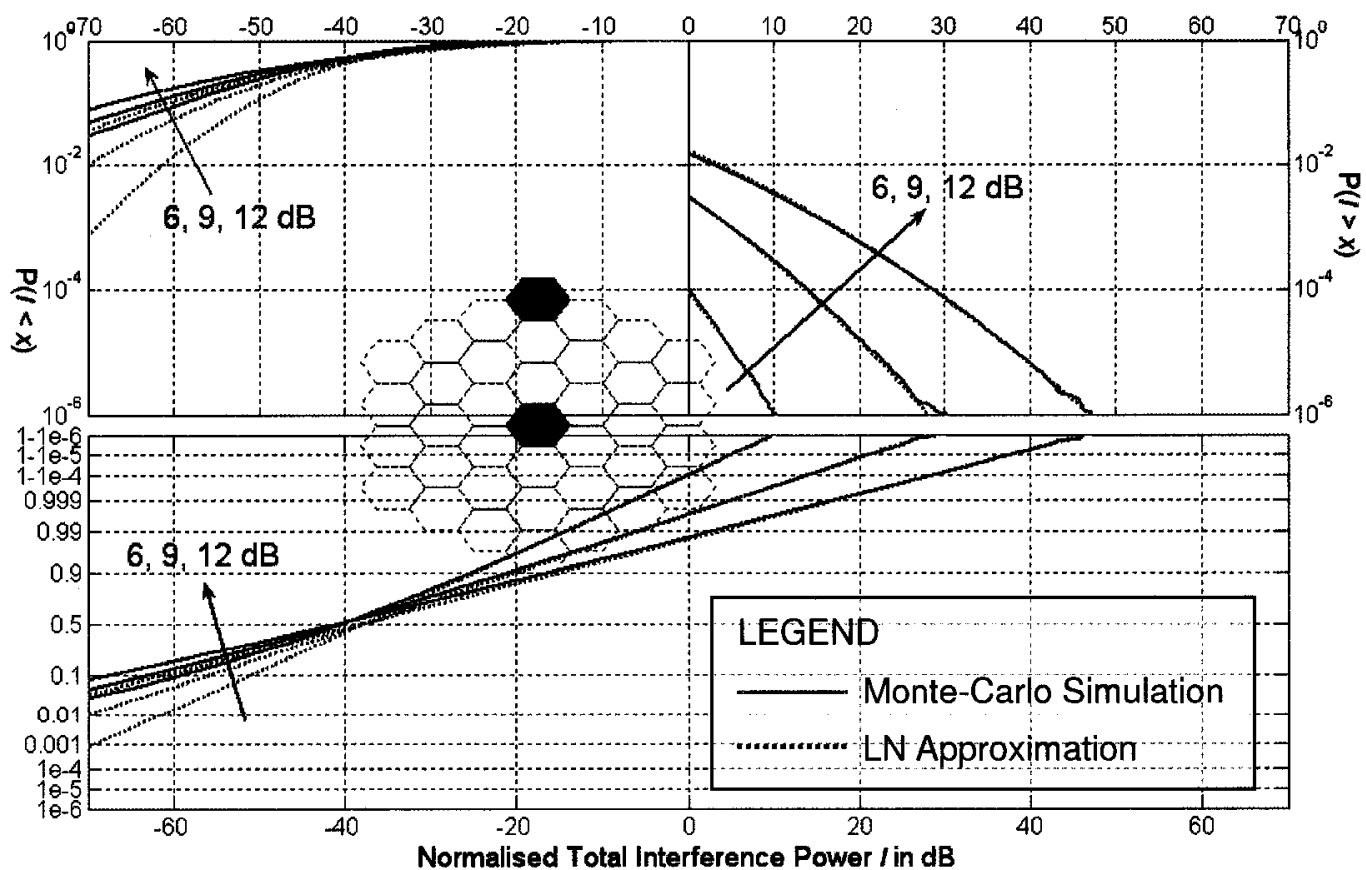

Figure 4-10 Single distant interferer, Rayleigh fading, uplink with power control. 


\subsection{Statistical Moments of Total Interference Power}

Although the moments (of natural order) do not entirely characterise a distribution [43], they nevertheless offer much useful information about it. In this section, we present both an exact expression for the first two moments of the total interference power $I$, as well as good approximations, which hold under the assumption that the shadowing variance is large enough, which we find to be the case in realistic scenarios $(6-12 \mathrm{~dB})$.

\subsubsection{First Moment}

\subsubsection{Uplink without Power Control, and Downlink}

Let us define $A_{n}$ coefficients, which are a function of the cellular layout and the propagation constant, as follows:

$$
A_{n}=E\left[\sum_{i=1}^{N} R_{i}^{n}\right]=\sum_{i=1}^{N} E\left[R_{i}^{n}\right]=\sum_{i=1}^{N} \kappa_{i}(n \beta)
$$

In the DL, the DMU receives interference from all FS's. The power transmitted by these is independent of the position of other users in the interfering cells. Similarly, in the UL - no PC case, the transmit power of each IMU is independent of its position. We thus conclude that each pathloss is independent of all other factors, and we may write:

$$
E[I]=E\left[\sum_{i=1}^{N} R_{i} Q_{i}\right]=\sum_{i=1}^{N} E\left[R_{i}\right] E\left[Q_{i}\right]=E\left[\sum_{i=1}^{N} R_{i}\right] E\left[Q_{i}\right]=A_{1} B_{1} \quad\left[k_{D L}, k_{P C}\right] \neq[0,1]
$$

\subsubsection{Uplink with Power Control}

The method needs to be modified slightly for the UL - PC case. We define: 


$$
\tilde{A}_{n}=E\left[\sum_{i=1}^{N} R_{i}^{n} L_{i}^{n}\right]=\sum_{i=1}^{N} E\left[R_{i}^{n} L_{i}^{n}\right]=\sum_{i=1}^{N} \widetilde{\kappa}_{i}(n \beta)
$$

Then:

$$
E[I]=\sum_{i=1}^{N} E\left[R_{i} L_{i}\right] E\left[\frac{Q_{i}}{L_{i}}\right]=E\left[\sum_{i=1}^{N} R_{i} L_{i}\right] E\left[\frac{Q_{i}}{L_{i}}\right]=\tilde{A}_{1} \tilde{B}_{1} \quad\left[k_{D L}, k_{P C}\right]=[0,1]
$$

\subsubsection{Second Moment}

\subsubsection{Uplink without Power Control}

$$
\begin{aligned}
& E\left[I^{2}\right]=E\left[\left(\sum_{i=1}^{N} I_{i}\right)^{2}\right]=\sum_{i=1}^{N} \sum_{j=1}^{N} E\left[I_{i} I_{j}\right]=\sum_{i=1}^{N} E\left[I_{i}^{2}\right]+\sum_{\substack{i=1 \\
j=1 \\
j \neq i}}^{N} E\left[I_{i} I_{j}\right] \\
& =\sum_{i=1}^{N} E\left[R_{i}^{2}\right] E\left[Q_{i}^{2}\right]+\sum_{i=1}^{N} \sum_{\substack{j=1 \\
j \neq i}}^{N} E\left[R_{i} R_{j}\right] E\left[Q_{i}\right] E\left[Q_{j}\right]=A_{2} B_{2}+B_{1}^{2} \sum_{\substack{i=1 \\
j}}^{N} \sum_{\substack{j \neq i \\
j \neq i}}^{N} E\left[R_{i} R_{j}\right] .
\end{aligned}
$$

In the UL, the pathlosses are independent, hence:

$$
\begin{aligned}
& E\left[I^{2}\right]=A_{2} B_{2}+\left(\sum_{i=1}^{N} \sum_{j=1}^{N} E\left[R_{i}\right] E\left[R_{j}\right]-\sum_{i=1}^{N} E\left[R_{i}^{2}\right]\right) B_{1}^{2} \\
& =A_{2} B_{2}+\left(\left(\sum_{i=1}^{N} \kappa_{i}(\beta)\right)^{2}-\sum_{i=1}^{N} \kappa_{i}(2 \beta)\right) B_{1}^{2}=A_{2} B_{2}+\left(A_{1}^{2}-A_{2}\right) B_{1}^{2} \quad k_{D L}=k_{P C}=0 .
\end{aligned}
$$

\subsubsection{Uplink with Power Control}

Analogically, we can find the moment in UL-PC:

$$
E\left[I^{2}\right]=\tilde{A}_{2} \tilde{B}_{2}+\left(\tilde{A}_{1}^{2}-\tilde{A}_{2}\right) \tilde{B}_{1}^{2} \quad\left[k_{D L}, k_{P C}\right]=[0,1]
$$

\subsubsection{Downlink}

Define: 


$$
A_{D L}=\sum_{i=1}^{N} \sum_{j=1}^{N} E\left[R_{i} R_{j}\right]=E\left[\left(\sum_{i=1}^{N} R_{i}\right)^{2}\right]
$$

In the DL, the distances between the DMU and the IFS's are not independent and $A_{D L}$ cannot be separated into a sum of terms, but must be integrated for the entire geometry as follows:

$$
A_{D L}=\frac{2}{3 \sqrt{3}} \iint_{(x, y) \in C_{0}}\left(\sum_{i=1}^{N}{\sqrt{\left(x-x_{i}\right)^{2}+\left(y-y_{i}\right)^{2}}}^{-\beta}\right)^{2} d x d y
$$

where $x_{i}, y_{i}$ and $C_{0}$ are the same as in (4.2). Then:

$$
E\left[I^{2}\right]=A_{2} B_{2}+\left(E\left[\sum_{i=1}^{N} \sum_{j=1}^{N} R_{i} R_{j}\right]-\sum_{i=1}^{N} E\left[R_{i}^{2}\right]\right) B_{1}^{2}=A_{2} B_{2}+\left(A_{D L}-A_{2}\right) B_{1}^{2} \quad k_{D L}=1
$$

\subsubsection{A Simple Approximation to Moments}

We found in our numerical examples that only the first term in the second moment expressions is significant, and that the following approximation is usually exact to several digits:

$$
E\left[I^{2}\right] \cong A_{2} B_{2} \quad \forall k_{D L}, \forall k_{P C}
$$

We conjecture that, in general,

$$
E\left[I^{n}\right] \cong A_{n} B_{n} \quad n=1,2,3 \ldots \quad \forall k_{D L}, \forall k_{P C}
$$

We believe the reason for this is that since $B_{n}$ is dominated by a LN RV factor with high variance, which has moments of the order of $e^{n^{2}}$, therefore $B_{n} \gg B_{n-1}$. If write the multinomial expansion of (4.33), we will find that there will be one term containing $B_{n}$, which is the one given by the equation, which will dominate. 
Notice that the expressions for the moments are then identical for UL and DL, which is suggested by [47].

\subsection{Modeling the Total Interference Power as Bernoulli Lognormal}

We approximate $I$ by a LN RV of parameters $\left(a_{l}, b_{I}{ }^{2}\right)$ multiplied by an independent Bernoulli RV with parameter $p_{\chi}$, giving a BLN distribution:

$$
\begin{gathered}
F_{I}(x) \cong \Phi\left(\frac{\ln x-a_{I}}{b_{I}}\right) p_{\chi}+\left(1-p_{\chi}\right), x>0, \\
p_{\chi}=P[I \neq 0]=1-(1-p)^{N} .
\end{gathered}
$$

\subsubsection{Arguments for the Choice of Distribution}

We have, according to the FW method, chosen to model a sum of (approximately) LN RV's by another LN RV. It is not self-evident that a linear combination of LN RV's with random weights (namely the Bernoulli RV's $\chi_{i}$ ), will again be LN. Nevertheless, in [1] this assumption is made. We can argue that for any realisation of the vector $\chi_{i}$, we have a sum of $\sum_{i=1}^{N} \chi_{i}$ interferers, which is itself approximately LN. The resulting $p d f$ is then the weighed sum of the $p d f$ 's for each realisation of the vector $\chi_{i}$, with the weights being the probability associated with each realisation. This is called a mixture of LN distributions; it is not known whether it is approximately LN. However we do know that because the individual distributions are smooth (LN), their sum will also be smooth, so a $\mathrm{LN}$ approximation to their sum is not inconceivable. We notice, however, that if the probability of activity $p$ is low, there is a chance of there being absolutely no interference if all $\chi_{i}=0$. This creates a discontinuity in the $c d f$ of $I$, which modifies the LN 
distribution into a BLN one in (4.34). In most cases, for many interferers and moderate to high $p$, we have $p_{\chi} \approx 1$ and the model becomes LN as in [1].

\subsubsection{Moment-Matching}

We match the moments of the interference with those of the approximating distribution:

$$
E\left[I^{n}\right]=p_{\chi} \cdot e^{n a_{I}+\frac{n^{2}}{2} b_{I}^{2}}
$$

Inverting (4.36), we obtain:

$$
\begin{aligned}
& a_{I}=2 \ln E[I]-\frac{1}{2} \ln E\left[I^{2}\right]-\frac{3}{2} \ln p_{\chi} \\
& b_{I}^{2}=-2 \ln E[I]+\ln E\left[I^{2}\right]+\ln p_{\chi}
\end{aligned}
$$

\subsubsection{Simple Approximations to the Distribution Parameters}

Applying the approximation (4.32) to equations (4.37) and (4.38), we obtain simple closed-form equations that are applicable to all cases:

$$
\begin{gathered}
a_{l} \cong 2 \ln A_{1} B_{1}-\frac{1}{2} \ln A_{2} B_{2}-\frac{3}{2} \ln \left(p_{\chi}\right) \\
=2 \ln A_{1}-\frac{1}{2} \ln A_{2}+\frac{3}{2} \ln \frac{p}{p_{\chi}}-\frac{1}{2} \ln \left(2-\left(1+K^{-1}\right)^{-2}\right), \\
+k_{P C}\left[\beta \ln \rho-2 \ln \left(1+\frac{1}{2} \beta\right)+\frac{1}{2} \ln (1+\beta)\right] \\
b_{I}^{2} \cong-2 \ln A_{1} B_{1}+\ln A_{2} B_{2}+\ln p_{\chi} \\
=\sigma^{2}-2 \ln A_{1}+\ln A_{2}-\ln \frac{p}{p_{\chi}}+\ln \left(2-\left(1+K^{-1}\right)^{-2}\right) . \\
+k_{P C}\left[\sigma^{2}+\sigma_{e}^{2}+2 \ln \left(1+\frac{1}{2} \beta\right)-\ln (1+\beta)\right]
\end{gathered}
$$

For the UL - PC case, this approximation simply treats $R_{j}$ and $L_{j}$ as independent, but with the same marginal distributions. We notice that the expressions are identical for UL and DL. A 
similar result was obtained in [47]. This is not entirely surprising, since the interfering power from each individual cell has identical distribution in UL and DL. Only in the DL case, the pathlosses $R_{j}$ are correlated. However, since these are further multiplied by i.i.d. LN RV's with high variance, the correlation between the individual powers becomes very small. In other words, because every path suffers from independent shadowing with large variance, this effectively "drowns out" the correlation between the pathlosses and makes them very nearly independent. Also, in the UL - PC case, the independent shadowing terms make the correlation between $R_{j}$ and $L_{j}$ insignificant.

\subsection{Modeling the Total Interference Power as Bernoulli Power Lognormal}

We approximate $I$ by a PLN RV of parameters $\left(m_{l}, s_{I}^{2}, t_{l}\right)$ multiplied by an independent Bernoulli $\mathrm{RV}$ with the same parameter $p_{\chi}$ as given by (4.35), resulting in a Bernoulli Power Lognormal (BPLN) distribution:

$$
F_{I}(x) \cong \Phi^{t_{I}}\left(\frac{\ln x-m_{I}}{s_{I}}\right) p_{\chi}+\left(1-p_{\chi}\right), x>0
$$

\subsubsection{Arguments for the Choice of Distribution}

Following the same reasoning as in Section 4.4.1, we modify the PLN approximation to the SLN by multiplying it by the same Bernoulli RV, resulting in the discontinuous model (4.41).

\subsubsection{Matching the Upper Tail Slope}

We have seen in Section 3.3.1.1 that we can match the slope of the upper tail (on LN paper) of the SLN and the PLN distributions, by taking the slope of the component with the highest mean from among the components of the highest variance. A few numerical examples can be used to verify that interference from the nearest cells has not only the highest mean power in $\mathrm{dB}$ (which 
is natural), but also the highest $\mathrm{dB}$ spread. This is expected since the distance from a point in cell $C_{0}$ to a point in a faraway cell cannot vary overmuch for different points in the cells. The variability is much stronger for nearby cells.

In order to match the upper tail slope, we find the $c d f$ of the interference coming from one of the nearest cells, given that the user activity is constant $(p=1)$, since the result is only valid for a sum of LN RV's, and not of BLN RV's. For $p<1$, we argue that a BLN $c d f$ is just a scaled version of a corresponding $\mathrm{LN} c d f$ in the upper tail, and thus, by Lemma 2, these have the same blf, since their ratio is finite in the tail.

\subsubsection{Uplink without Power Control, and Downlink}

We first define the convenient intermediary quantity:

$$
\begin{aligned}
& z_{I}=\ln \frac{B_{2}}{p}-2 \ln \frac{B_{1}}{p}=\sigma^{2}+\ln \left(2-\left(1+K^{-1}\right)^{-2}\right) \\
& +k_{P C}\left[\sigma^{2}+\sigma_{e}^{2}+2 \ln \left(1+\frac{1}{2} \beta\right)-\ln (1+\beta)\right] .
\end{aligned}
$$

We call $C_{1}$ a nearest cell to $C_{0}$, and we can find the logarithmic variance of the interference component coming from this nearest cell by applying (4.21) to (3.48):

$$
s_{I}^{2}=b_{1}^{2}=\ln \kappa_{1}(2 \beta)-2 \ln \kappa_{1}(\beta)+z_{I} \quad\left[k_{D L}, k_{P C}\right] \neq[0,1]
$$

\subsubsection{Uplink with Power Control}

Analogically, for UL - PC: 


$$
\begin{gathered}
\tilde{z}_{I}=\ln \frac{\tilde{B}_{2}}{p}-2 \ln \frac{\tilde{B}_{1}}{p}=2 \sigma^{2}+\sigma_{e}^{2}+\ln \left(2-\left(1+K^{-1}\right)^{-2}\right), \\
s_{I}^{2}=\ln \tilde{\kappa}_{1}(2 \beta)-2 \ln \tilde{\kappa}_{1}(\beta)+\tilde{z}_{I} \quad\left[k_{D L}, k_{P C}\right]=[0,1] .
\end{gathered}
$$

\subsubsection{Matching the Lower Tail Slope}

\subsubsection{A Simple Approximation for All Cases}

If all components are i.i.d., the PLN approximation to the SLN uses $N$ as its power parameter ,as in [44]. Even if the components have different distribution, we find that this choice of parameter gives a good fit to the lower tail slope. However, if the individual components are multiplied by Bernoulli RV's with parameter $p$, then there are, on average, only $p N$ interferers, which is the idea in [3]. We can thus write:

$$
t_{I} \approx p N, \quad t_{I} \geq p N
$$

We proceed to show in the next section that this approximation is good.

\subsubsection{Uplink without Power Control, and Downlink}

A more exact approach to finding the power parameter of the PLN is to use (3.49), which equates the power parameter with $b_{1}^{2} \sum_{i=1}^{N} \frac{1}{b_{i}^{2}}$. By applying (4.21) and multiplying by $p$, as was done in (4.46), we obtain:

$$
t_{I}=p s_{I}^{2} \sum_{i=1}^{N} \frac{1}{\ln \kappa_{i}(2 \beta)-2 \ln \kappa_{i}(\beta)+z_{I}} \quad\left[k_{D L}, k_{P C}\right] \neq[0,1]
$$

where $s_{I}^{2}$ is given by (4.43).

We rewrite (4.47) as 


$$
t_{I}=p \sum_{i=1}^{N} \frac{\ln \kappa_{1}(2 \beta)-2 \ln \kappa_{1}(\beta)+z_{I}}{\ln \kappa_{i}(2 \beta)-2 \ln \kappa_{i}(\beta)+z_{I}} \quad\left[k_{D L}, k_{P C}\right] \neq[0,1]
$$

and we observe, from numerical calculations, that:

$$
\forall i: \ln \kappa_{i}(2 \beta)-2 \ln \kappa_{i}(\beta) \leq \ln \kappa_{1}(2 \beta)-2 \ln \kappa_{1}(\beta)<z_{I}
$$

Applying (4.49) to (4.48), we obtain the approximation in (4.46).

\subsubsection{Uplink with Power Control}

Likewise, for the UL - PC case, we have:

$$
t_{I}=p s_{I}^{2} \sum_{i=1}^{N} \frac{1}{\ln \tilde{\kappa}_{i}(2 \beta)-2 \ln \widetilde{\kappa}_{i}(\beta)+\tilde{z}_{I}} \quad\left[k_{D L}, k_{P C}\right]=[0,1]
$$

where $s_{I}^{2}$ is given by (4.45).

\subsubsection{Moment-Matching}

Having matched the slopes at both tails, we have only one more degree of freedom. We match the first moment of the PLN approximation with that of the interference, given by (4.25).

\subsubsection{Uplink without Power Control, and Downlink}

Matching the moments gives:

$$
E I=e^{m_{I}} \Lambda\left(s_{I}, t_{I}\right) p_{\chi}=A_{1} B_{1} \quad\left[k_{D L}, k_{P C}\right] \neq[0,1]
$$

where, the $\Lambda$ function is given by (3.50). We proceed as in Section 3.3.1.2 to find $m_{l}$ : 


$$
\begin{gathered}
m_{I}=\ln A_{1} B_{1}-\ln \Lambda\left(s_{I}, t_{I}\right) p_{z}=\frac{1}{2} \sigma^{2}+\ln A_{1}-\ln \Lambda\left(s_{I}, t_{I}\right) \\
+\ln \frac{p}{p_{\chi}}+k_{P C}\left[\frac{1}{2} \sigma^{2}+\frac{1}{2} \sigma_{e}^{2}+\beta \ln \rho-\ln \left(1+\frac{1}{2} \beta\right)\right] \quad\left[k_{D L}, k_{P C}\right] \neq[0,1] .
\end{gathered}
$$

\subsubsection{Uplink with Power Control}

Likewise, for UL - PC:

$$
\begin{gathered}
E I=e^{m_{I}} \Lambda\left(s_{I}, t_{I}\right) p_{\chi}=\tilde{A}_{1} \tilde{B}_{1} \quad\left[k_{D L}, k_{P C}\right]=[0,1] \\
m_{I}=\ln \tilde{A}_{1} \tilde{B}_{1}-\ln \Lambda\left(s_{I}, t_{I}\right) p_{\chi} \\
=\sigma^{2}+\frac{1}{2} \sigma_{e}^{2}+\ln \tilde{A}_{1}-\ln \Lambda\left(s_{I}, t_{I}\right)+\ln \frac{p}{p_{\chi}} \quad\left[k_{D L}, k_{P C}\right]=[0,1] .
\end{gathered}
$$

\subsection{Comparison of Simulation, BLN and BPLN Methods}

\subsubsection{Cellular Layouts and their Coefficients}

Tables 4-6 to 4-10 have the geometry coefficients tabulated against $\beta$ for five different cellular layouts: a single tier of nearest interferers (1T), three tiers of interferers in a reuse 1 cellular network (3T), a reuse 3 cellular network $(R 3)$, a reuse 7 cellular network $(R 7)$, and a random layout $(R D)$ to test the robustness of our method. Only the $A_{D L}$ coefficients are really new information, and need to be integrated as per (4.30). All other coefficients can be found from Tables 4-1 to 4-4 by summing over the individual cells using (4.22) and (4.24). 


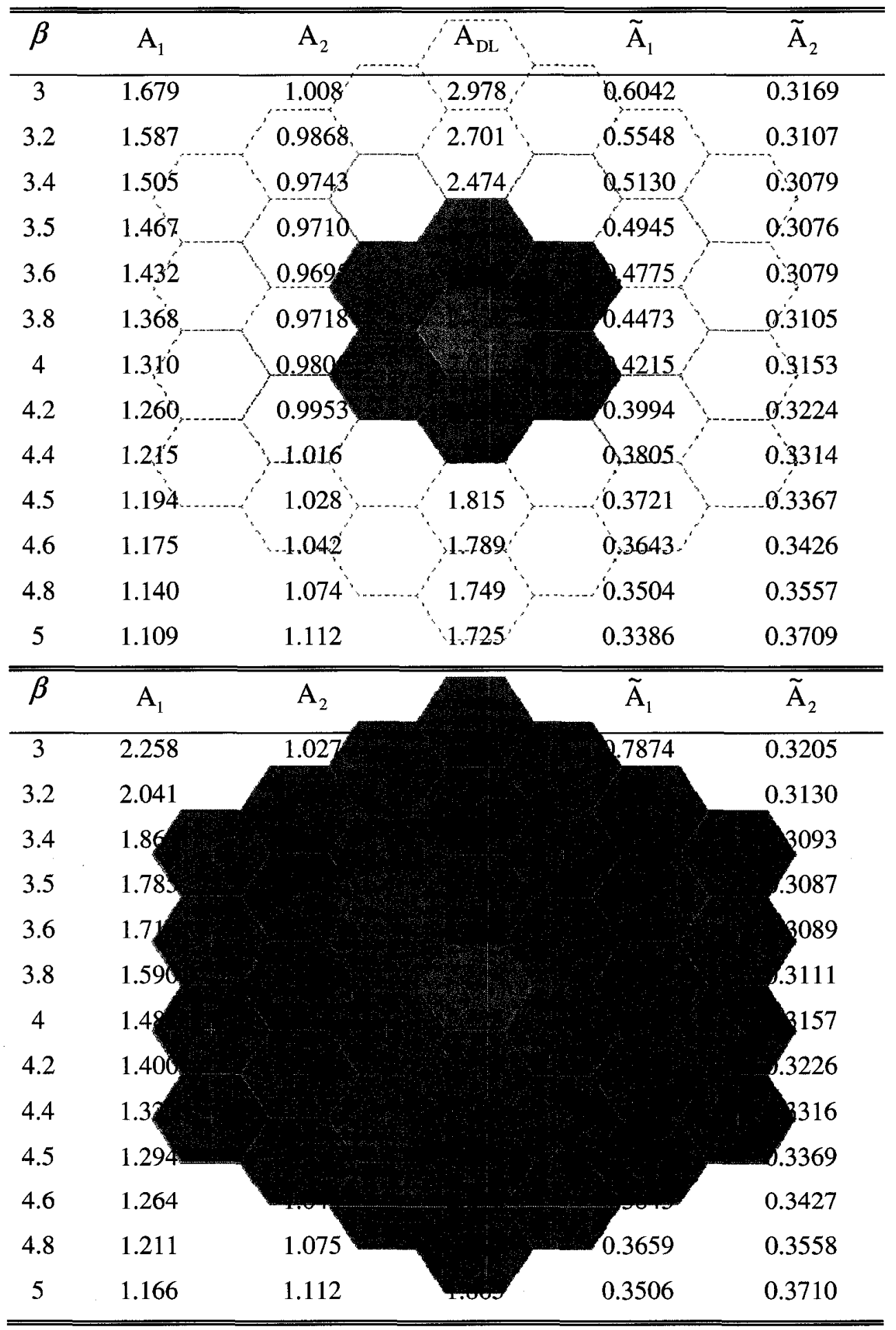

Table 4-6 Geometry parameters for various cellular layouts. 


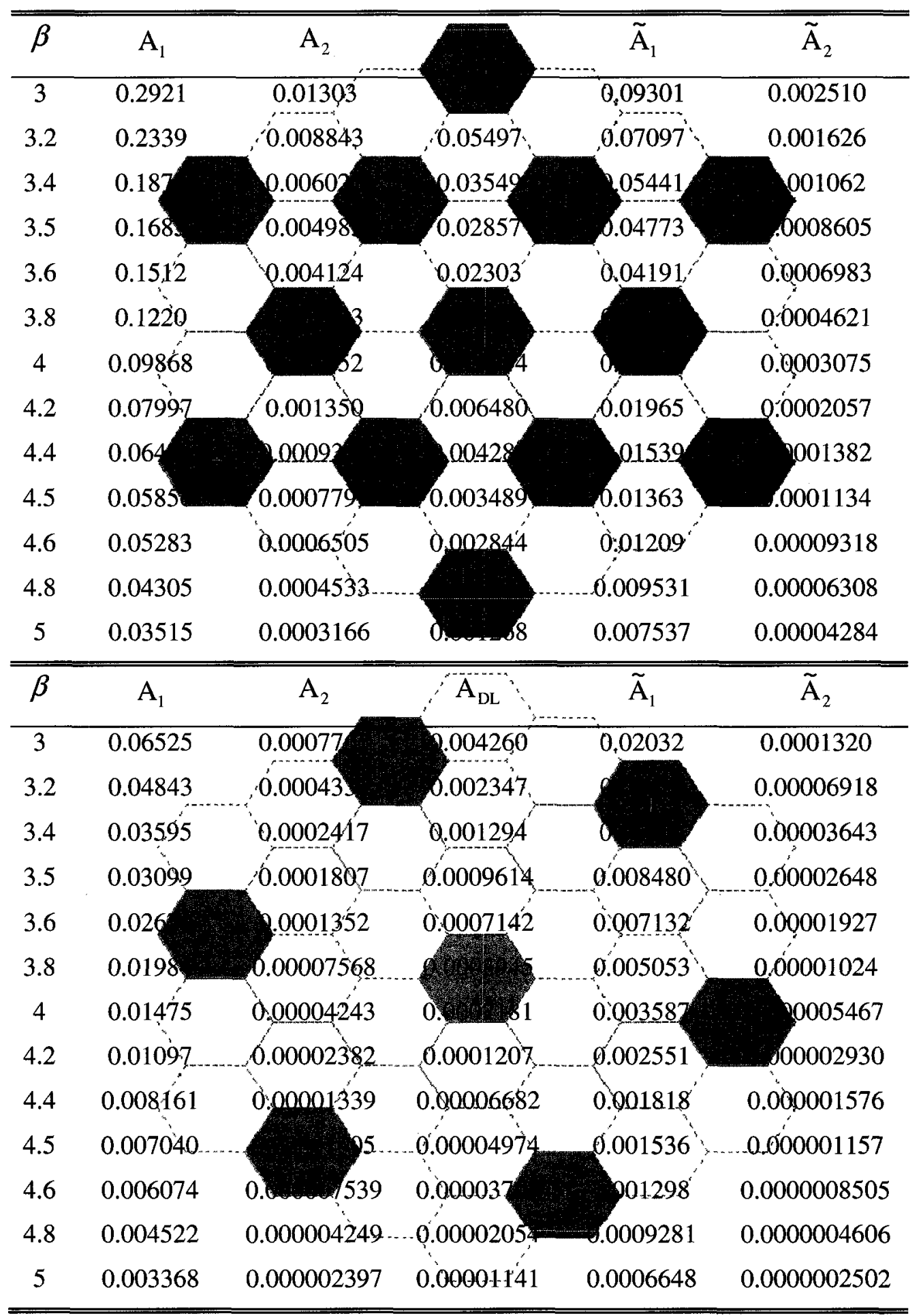

Table 4-6 (continued) 


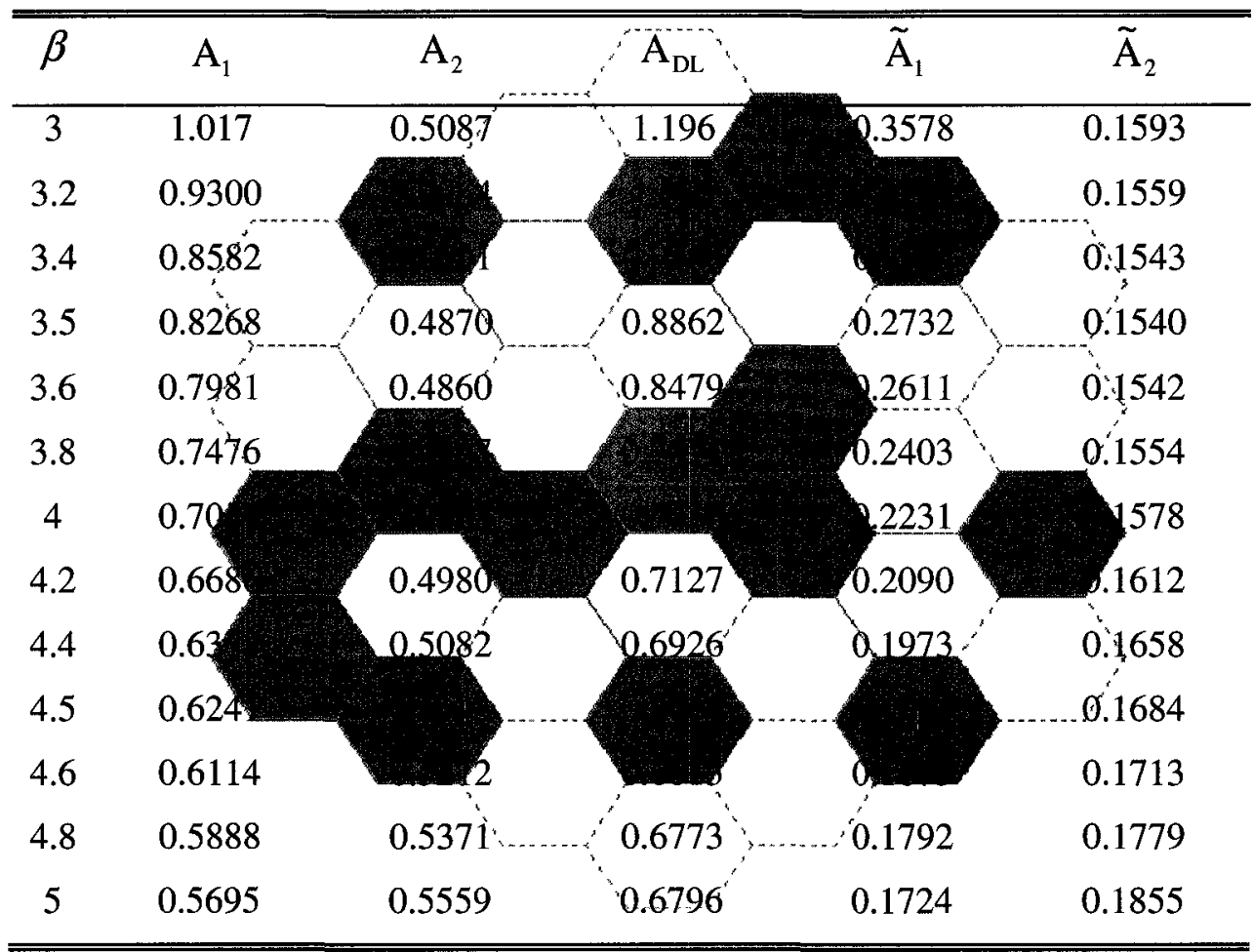

Table 4-6 (continued)

\subsubsection{Simulated and Analytical Parameters and Graphs}

We run simulations according to the algorithm in Figures 4-2 and 4-3. The input parameters are given in Table 4-7 while $\sigma_{e}=1 \mathrm{~dB} \approx 0.23$. Figures $4-11$ to $4-19$ show the results of the simulations, as well as the analytical approximations.

1. The exact BLN method uses (4.27), (4.28), or (4.31) in (4.37) and (4.38) to obtain the exact LN parameters $a_{l}$ and $b_{l}$.

2. The approximate BLN method uses (4.39) and (4.40) to obtain approximate values of these parameters.

3. Both the exact and approximate BPLN methods use (4.42) and (4.43), or (4.44) and (4.45) to find the first parameter $s_{I}$. 
4. The approximate BPLN method uses (4.46) to find $t_{1}$.

5. The exact BPLN method uses (4.42) and (4.47), or (4.44) and (4.50) to find $t_{r}$.

6. Both the exact and approximate methods use (4.52) or (4.54) to find $m_{l}$, where the integral $\Lambda\left(s_{I}, t_{I}\right)$ is calculated numerically as per (3.50).

\begin{tabular}{|c|c|c|c|c|c|c|c|c|}
\hline Figure & \multicolumn{3}{|c|}{$4-11$} & \multicolumn{2}{|c|}{$4-12$} & \multicolumn{3}{|c|}{$4-13$} \\
\hline$\beta$ & & 4 & & \multicolumn{2}{|c|}{4} & \multicolumn{3}{|c|}{3} \\
\hline$\Sigma[\mathrm{dB}]$ & & 12 & & \multicolumn{2}{|c|}{12} & 6 & 9 & 12 \\
\hline $\boldsymbol{K}$ & & 0 & & \multicolumn{2}{|c|}{0} & & 0 & \\
\hline$p$ & 0.01 & 0.1 & 1 & \multicolumn{2}{|c|}{1} & & 1 & \\
\hline Layout & & $3 T$ & & $1 T$ & $3 T$ & & $1 T$ & \\
\hline Link & & DL & & \multicolumn{2}{|c|}{ DL } & \multicolumn{3}{|c|}{ UL } \\
\hline PC & & No & & \multicolumn{2}{|c|}{ Yes } & \multicolumn{3}{|c|}{ No } \\
\hline $\boldsymbol{p}_{\chi}$ & 0.304 & 0.977 & 1 & \multicolumn{2}{|c|}{1} & \multicolumn{3}{|c|}{1} \\
\hline$a_{I}=$ & -4.665 & -2.965 & 0.455 & -1.542 & -1.293 & 0.624 & 0.680 & 0.686 \\
\hline$a_{I} \approx$ & -4.665 & -2.965 & 0.455 & -1.542 & -1.293 & 0.686 & 0.686 & 0.686 \\
\hline$b_{I}=$ & 3.308 & 3.132 & 2.744 & 4.007 & 3.976 & 1.306 & 1.995 & 2.703 \\
\hline$b_{I} \approx$ & 3.308 & 3.132 & 2.744 & 3.962 & 3.931 & 1.258 & 1.992 & 2.703 \\
\hline$m_{I}=$ & NA & -4.183 & -4.026 & -4.224 & -5.915 & -1.666 & -1.837 & -1.937 \\
\hline$m_{I} \approx$ & NA & -4.092 & -3.942 & -4.224 & -5.866 & -1.666 & -1.837 & -1.937 \\
\hline$s_{I}$ & NA & \multicolumn{2}{|c|}{3.094} & \multicolumn{2}{|c|}{4.225} & 1.837 & 2.400 & 3.017 \\
\hline$t_{I}=$ & NA & 3.960 & 39.60 & 6 & 37.83 & & 6 & \\
\hline$t_{I} \approx$ & NA & 3.6 & 36 & 6 & 36 & & 6 & \\
\hline
\end{tabular}

Table 4-7 Simulation and analytical parameters for BLN and BPLN methods. 


\begin{tabular}{|c|c|c|c|c|c|c|c|c|}
\hline Figure & $\overline{44-14}$ & \multicolumn{4}{|c|}{$4-15$} & \multicolumn{3}{|c|}{$4-16$} \\
\hline$\beta$ & 3.5 & \multicolumn{4}{|c|}{4} & \multicolumn{3}{|c|}{4} \\
\hline$\sigma[\mathrm{dB}]$ & 10 & \multicolumn{4}{|c|}{12} & \multicolumn{3}{|c|}{12} \\
\hline $\boldsymbol{K}$ & 0 & & & & & 0 & 10 & $\infty$ \\
\hline$p$ & 1 & 0.02 & 0.1 & 0.5 & 1 & & 1 & \\
\hline Layout & $R 7$ & \multicolumn{4}{|c|}{$R 3$} & \multicolumn{3}{|c|}{$I T$} \\
\hline Link & UL & \multicolumn{4}{|c|}{$\mathrm{DL}$} & \multicolumn{3}{|c|}{$\mathrm{DL}$} \\
\hline $\mathbf{P C}$ & No & \multicolumn{4}{|c|}{ Yes } & \multicolumn{3}{|c|}{ Yes } \\
\hline$p_{\chi}$ & 1 & 0.215 & 0.718 & 0.99976 & 1 & & 1 & \\
\hline$a_{I}=$ & -2.991 & -7.171 & -6.563 & -4.646 & -3.607 & -1.542 & -1.276 & -1.196 \\
\hline$a_{I} \approx$ & -2.986 & -7.171 & -6.563 & -4.646 & -3.607 & -1.542 & -1.276 & -1.196 \\
\hline$b_{I}=$ & 2.082 & 4.168 & 4.120 & 3.961 & 3.873 & 4.007 & 3.940 & 3.919 \\
\hline$b_{I} \approx$ & 2.080 & 4.126 & 4.076 & 3.916 & 3.827 & 3.962 & 3.894 & 3.874 \\
\hline$m_{I}=$ & -5.525 & NA & -6.752 & -7.076 & -7.068 & -4.224 & -3.956 & -3.876 \\
\hline$m_{I} \approx$ & -5.525 & NA & -6.744 & -7.068 & -7.061 & -4.224 & -3.956 & -3.876 \\
\hline$s_{I}$ & 2.473 & NA & & 4.119 & & 4.225 & 4.161 & 4.142 \\
\hline$t_{I}=$ & 6 & $\mathrm{NA}$ & 1.209 & 6.043 & 12.09 & & 6 & \\
\hline$t_{I} \approx$ & 6 & NA & 1.2 & 6 & 12 & & 6 & \\
\hline
\end{tabular}

Table 4-7 (continued) 


\begin{tabular}{|c|c|c|c|c|c|c|c|}
\hline Figure & \multicolumn{2}{|c|}{ 4-17 } & \multicolumn{4}{|c|}{$4-18$} & $4-19$ \\
\hline$\beta$ & 3 & 4 & \multicolumn{4}{|c|}{3.5} & 4 \\
\hline$\sigma[\mathrm{dB}]$ & 12 & 6 & \multicolumn{4}{|c|}{8} & 12 \\
\hline K & $\infty$ & 0 & \multicolumn{4}{|c|}{0} & 5 \\
\hline$p$ & \multicolumn{2}{|c|}{1} & 0.02 & 0.1 & 0.5 & 1 & 1 \\
\hline Layout & \multicolumn{2}{|c|}{$1 T$} & \multicolumn{4}{|c|}{$R 3$} & $R D$ \\
\hline Link & \multicolumn{2}{|c|}{ UL } & \multicolumn{4}{|c|}{ UL } & DL \\
\hline PC & \multicolumn{2}{|c|}{ Yes } & \multicolumn{4}{|c|}{ Yes } & $\mathrm{PC}$ \\
\hline$p_{\chi}$ & \multicolumn{2}{|c|}{1} & 0.215 & 0.718 & 0.99975 & 1 & 1 \\
\hline$a_{I}=$ & -0.394 & -1.438 & -6.431 & -5.822 & -3.906 & -2.867 & -2.224 \\
\hline$a_{I} \approx$ & -0.373 & -1.542 & -6.404 & -5.795 & -3.879 & -2.839 & -2.224 \\
\hline$b_{I}=$ & 3.891 & 2.253 & 2.983 & 2.914 & 2.686 & 2.554 & 4.022 \\
\hline$b_{I} \approx$ & 3.806 & 2.061 & 2.895 & 2.824 & 2.588 & 2.450 & 3.978 \\
\hline$m_{I}=$ & -3.073 & -4.007 & NA & -6.041 & -6.300 & -6.244 & -5.517 \\
\hline$m_{I} \approx$ & -3.073 & -4.007 & NA & -6.024 & -6.284 & -6.228 & -5.469 \\
\hline$s_{I}$ & 4.115 & 2.621 & NA & 2.922 & 2.922 & 2.922 & 4.174 \\
\hline$t_{I}=$ & \multicolumn{2}{|c|}{6} & NA & 1.221 & 6.107 & 12.21 & 14.70 \\
\hline$t_{I} \approx$ & \multicolumn{2}{|c|}{6} & NA & 1.2 & 6 & 12 & 14 \\
\hline
\end{tabular}

Table 4-7 (continued) 


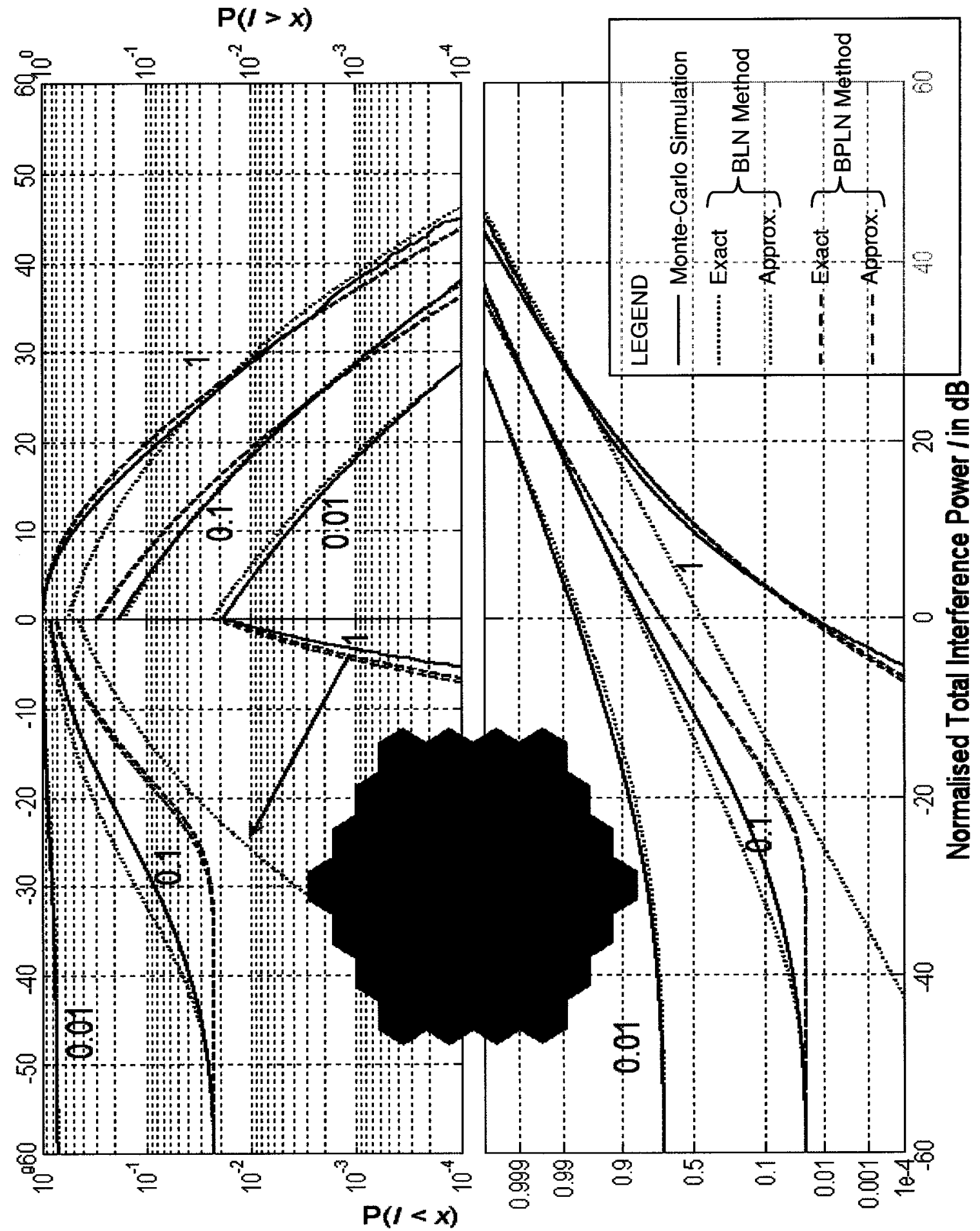

Figure 4-11 Interference in three-tier cellular network with variable activity in the downlink without power control. 


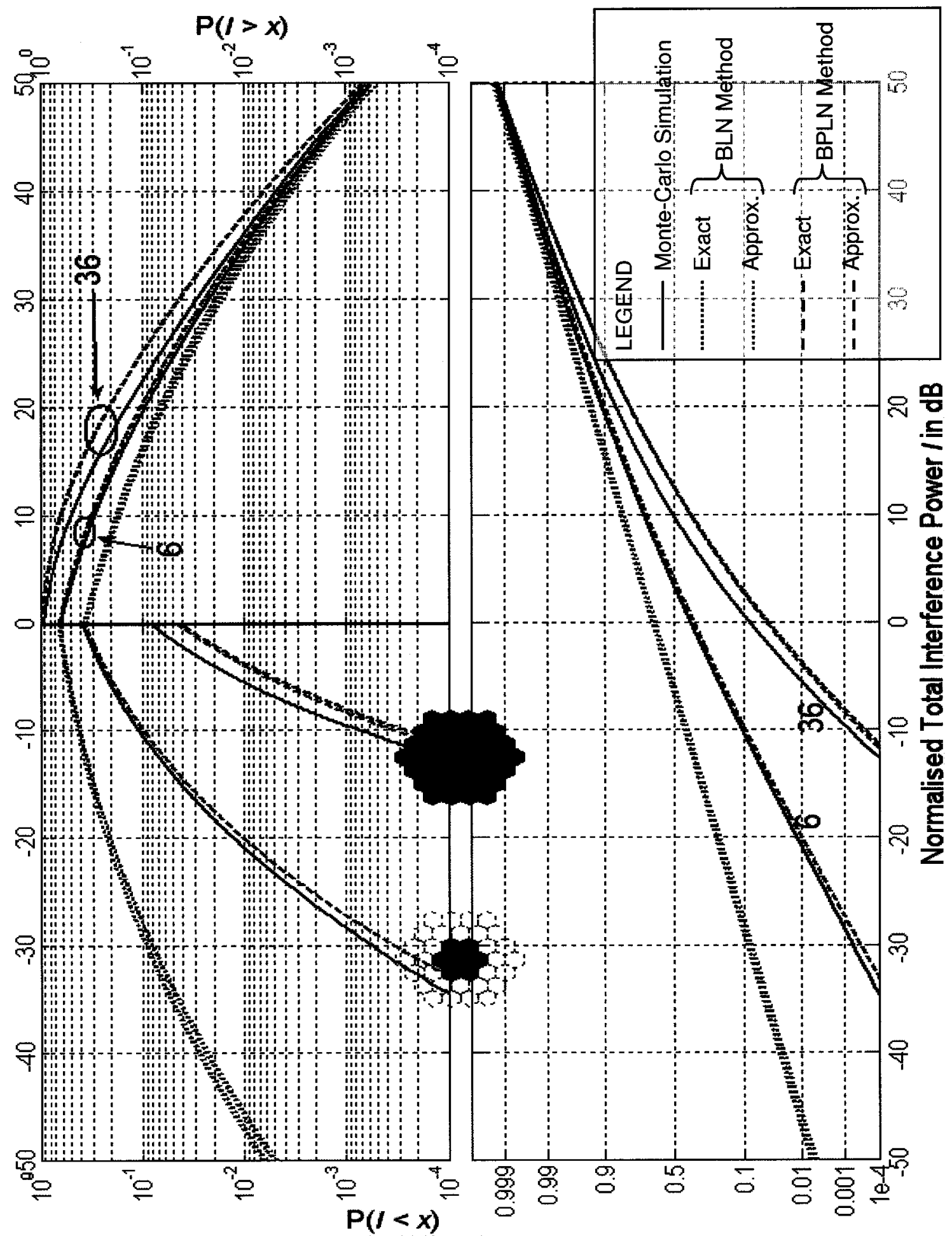

Figure 4-12 Comparison of interference from one and three tiers in the downlink with power control. 


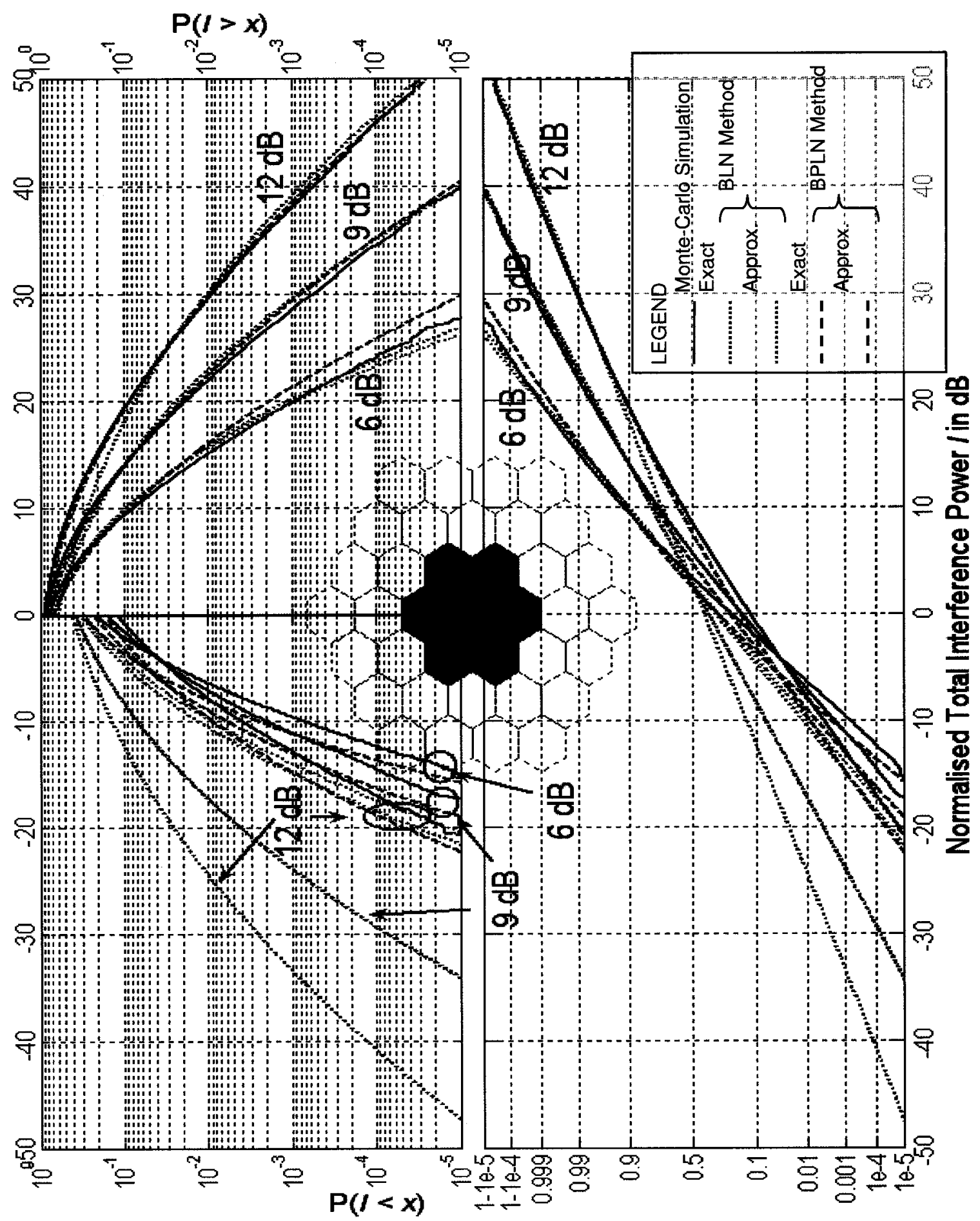

Figure 4-13 Effect of varying the shadowing parameter on interference from first tier in the uplink without power control. 


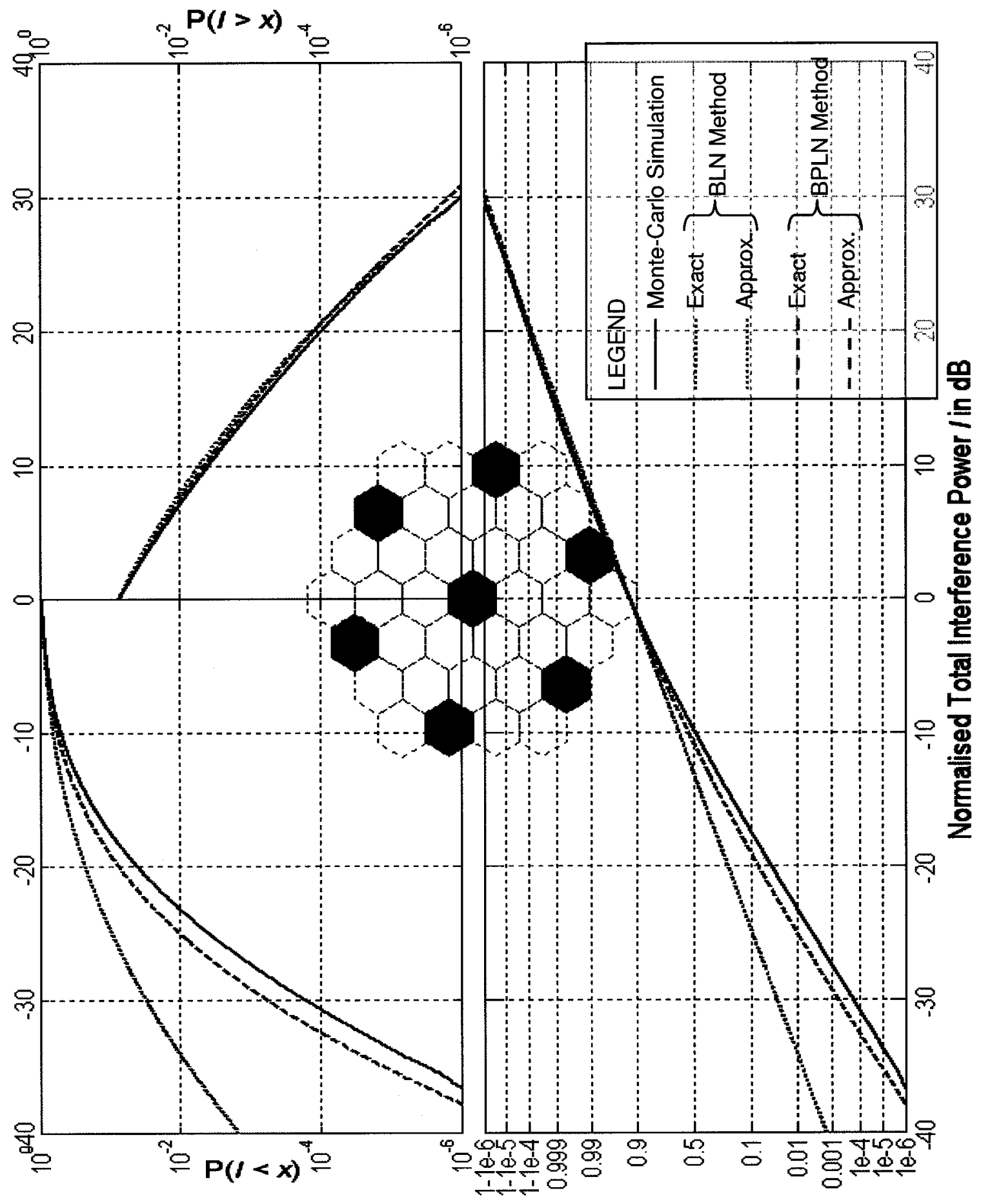

Figure 4-14 Interference in a reuse 7 cellular network in the uplink without power control. 


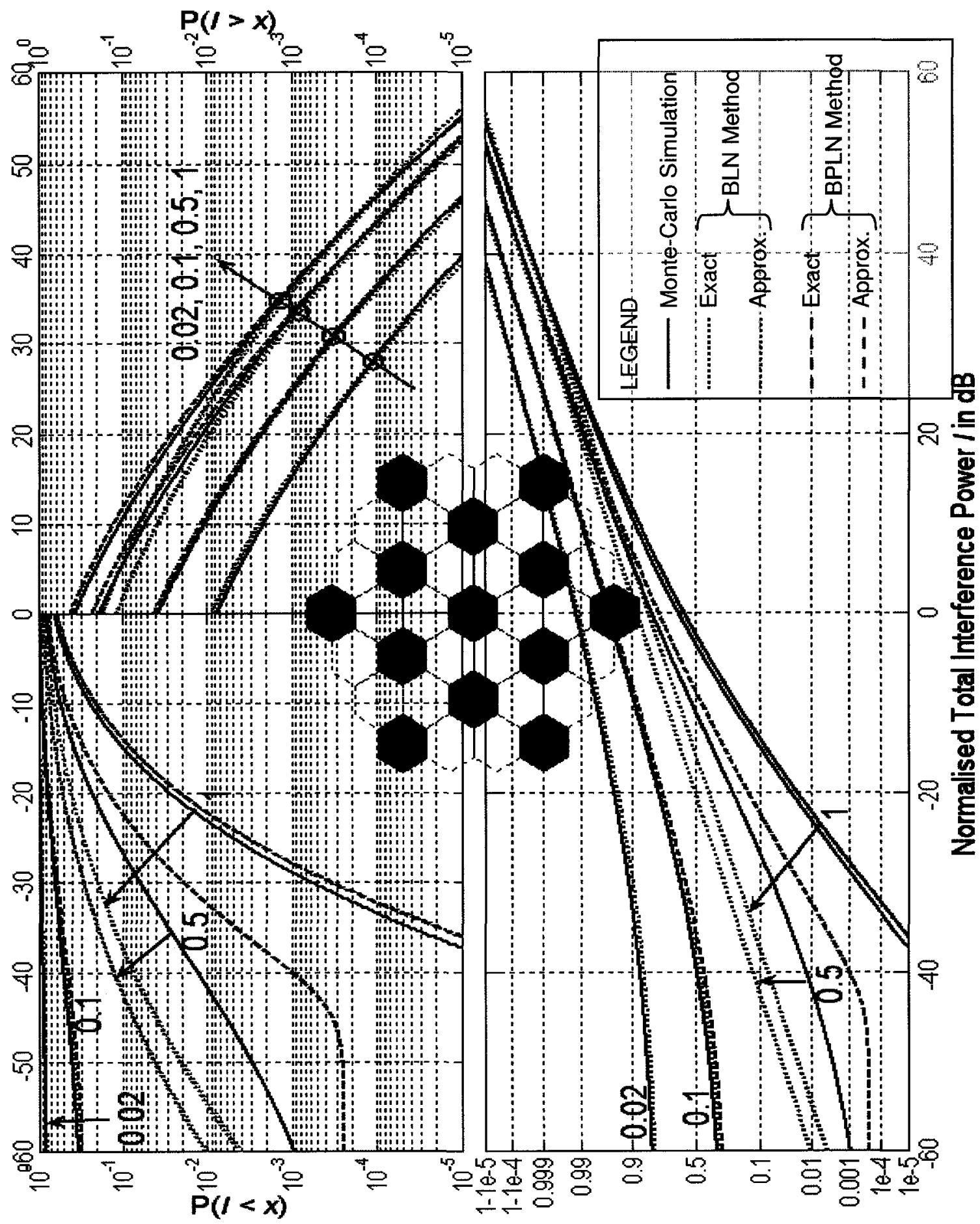

Figure 4-15 Interference in reuse 3 cellular network with variable activity level in the downlink with power control. 


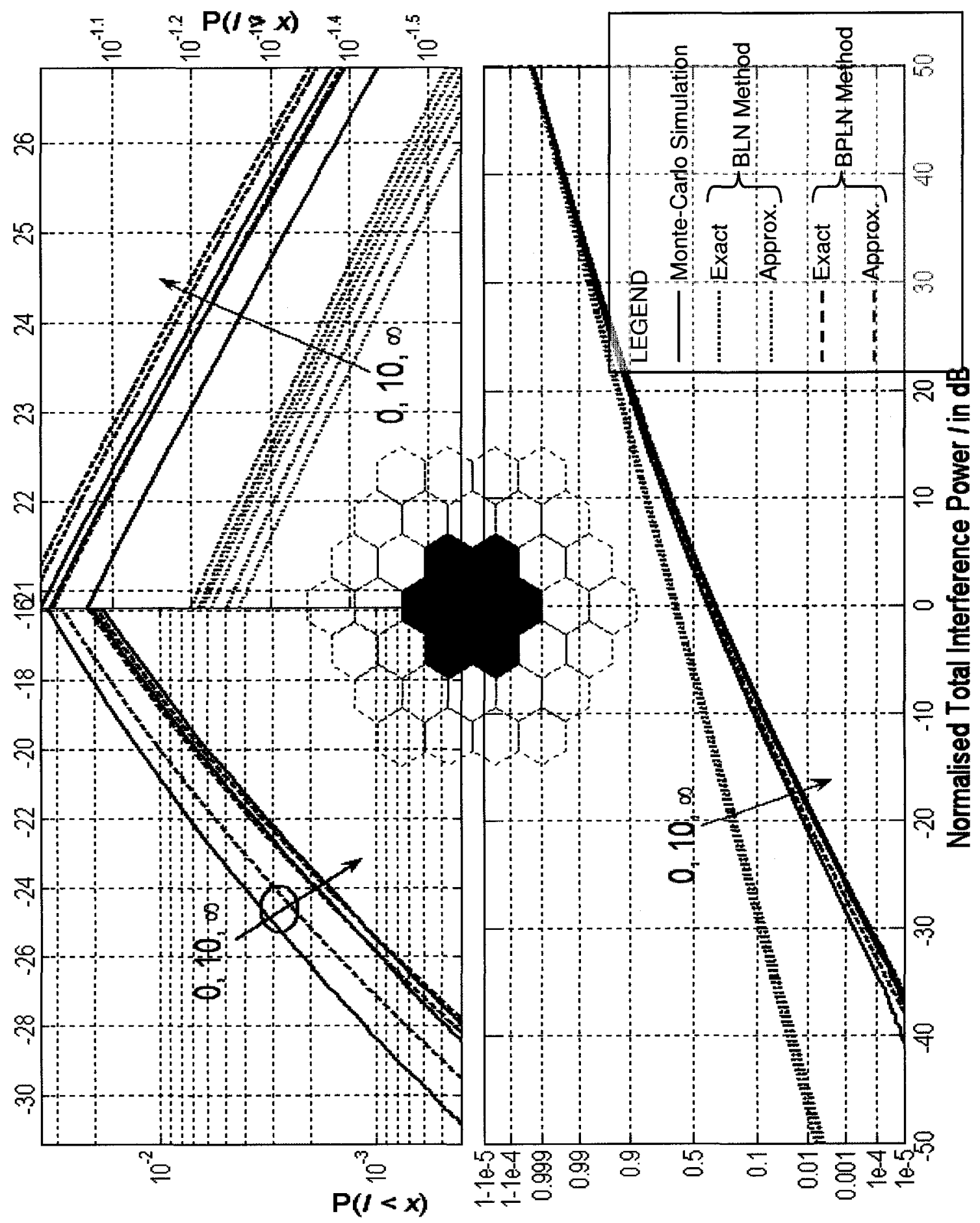

Figure 4-16 Interference from first tier with Rayleigh, Rician and no fading in the downlink with power control. 


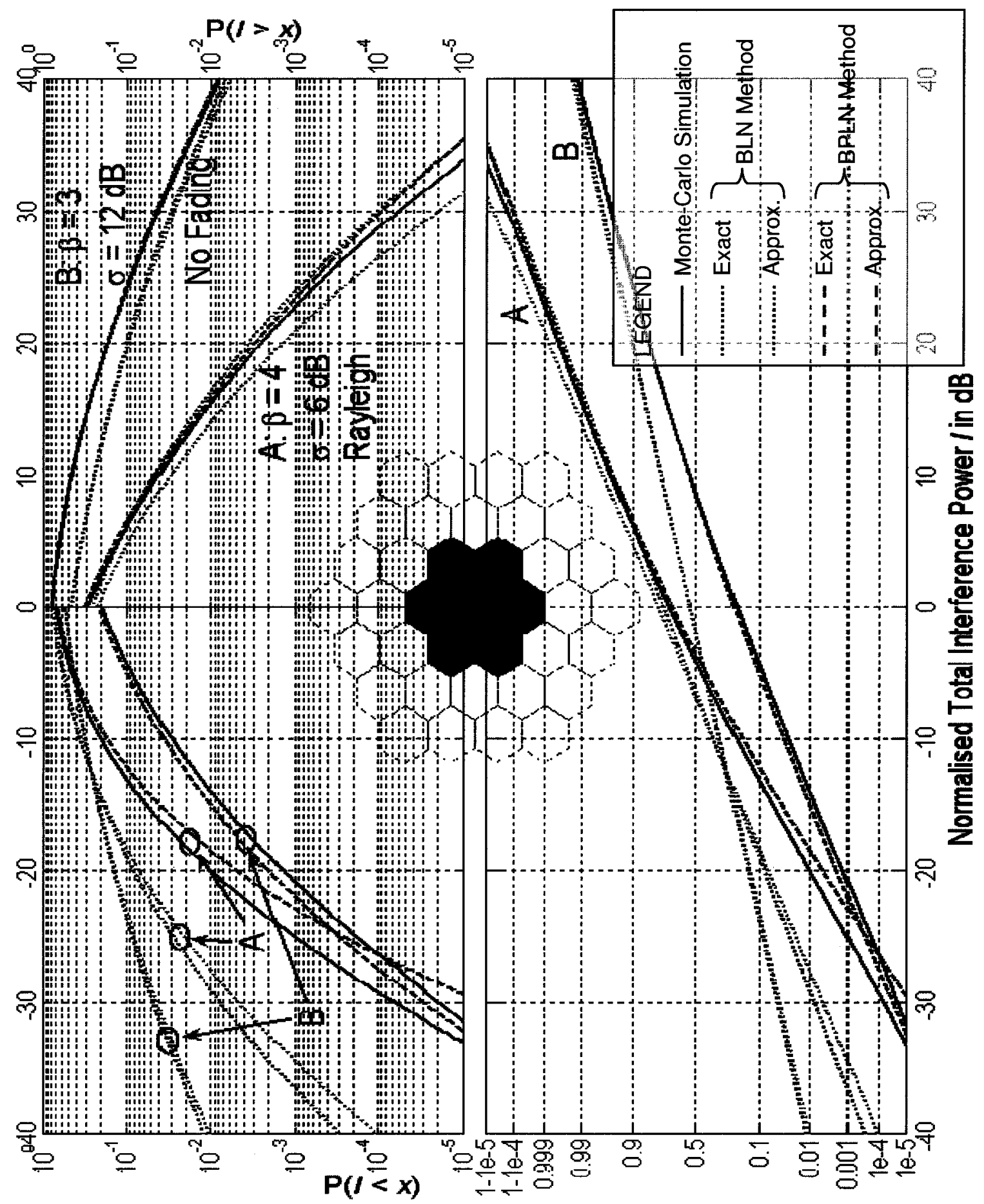

Figure 4-17 Interference from first tier while varying propagation exponent, shadowing and fading in the uplink with power control. 


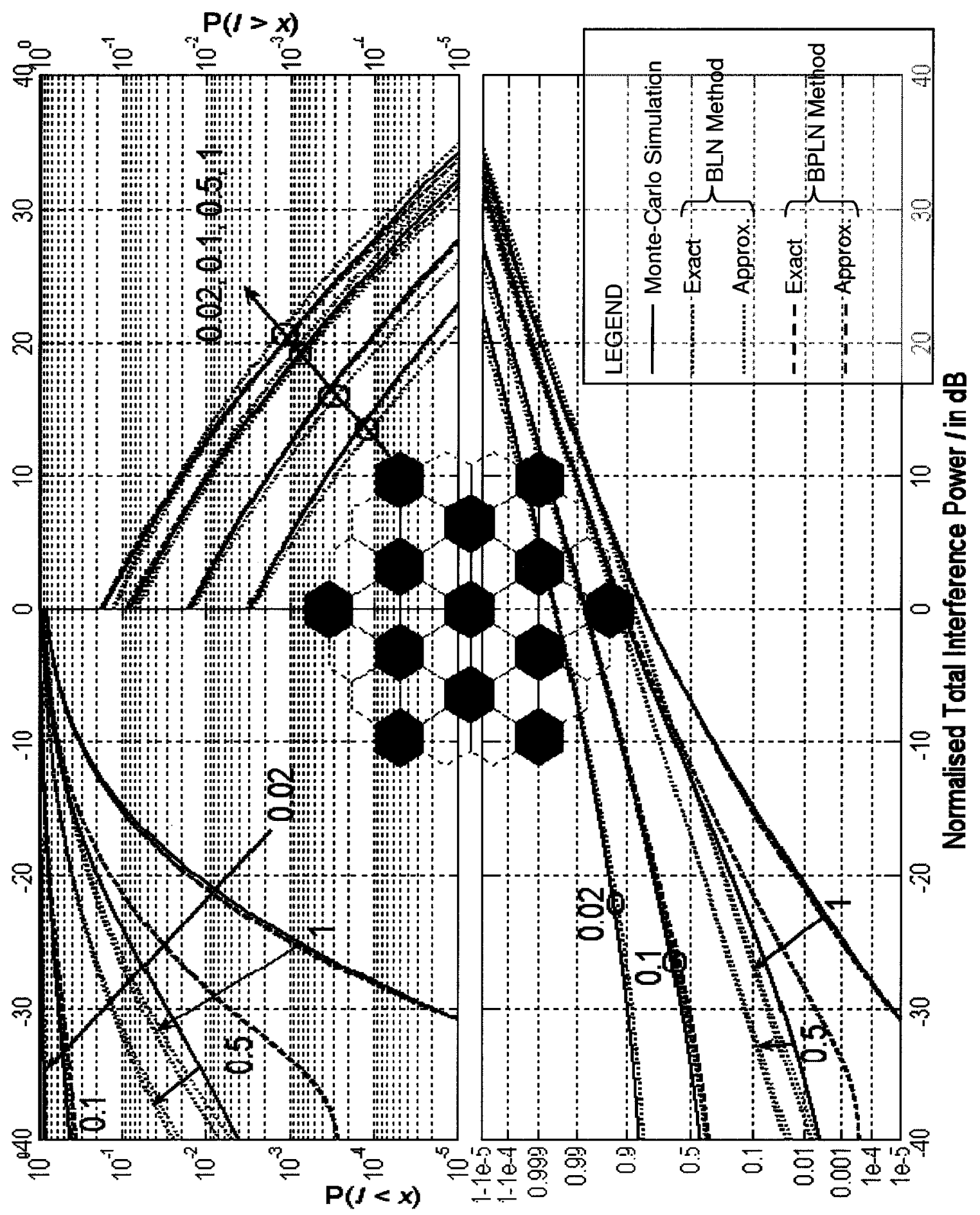

Figure 4-18 Interference in reuse 3 cellular network with variable activity level in the uplink with power control. 


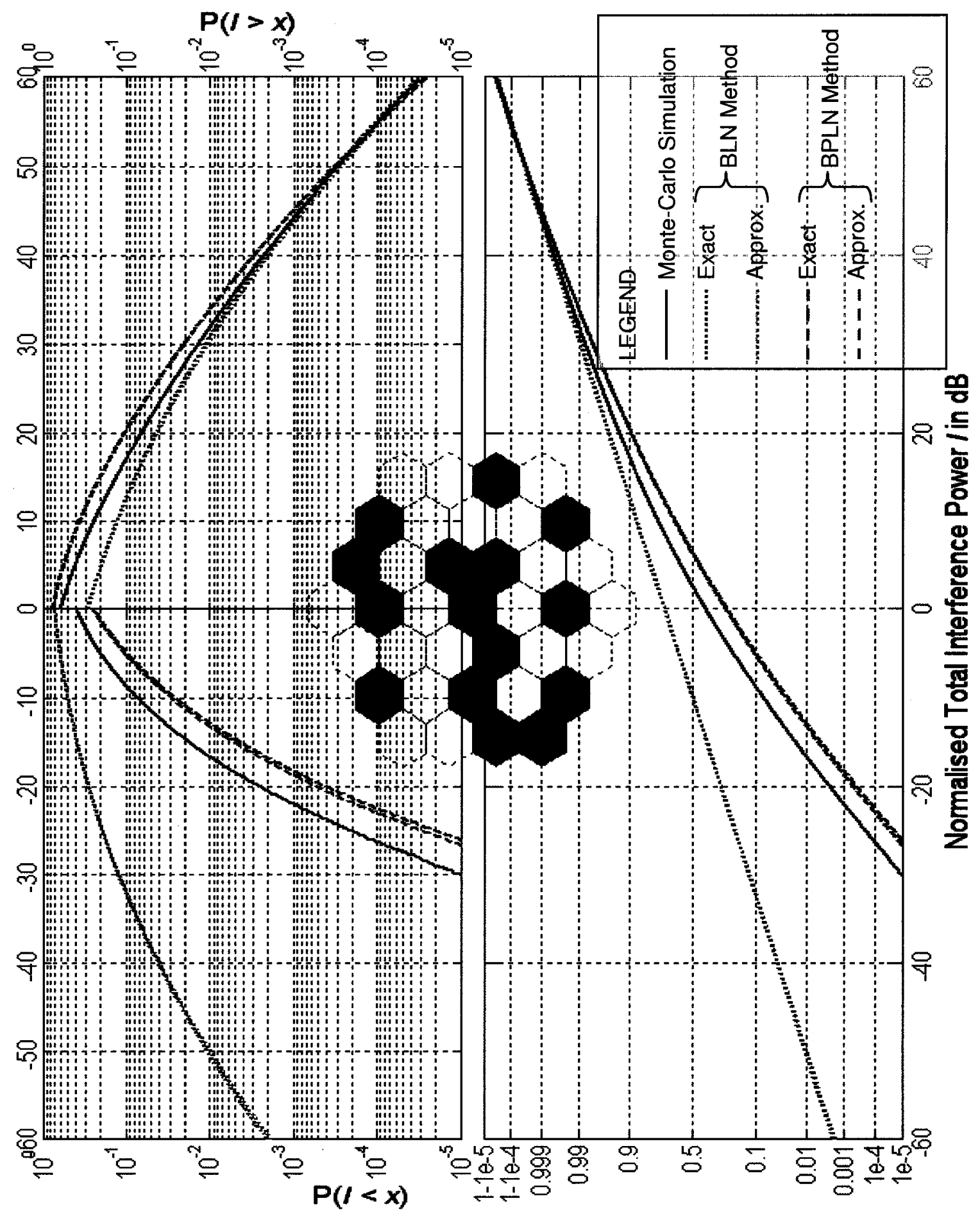

Figure 4-19 Interference from a random layout of cells in the uplink with power control. 


\subsubsection{Discussion on Observed Results}

Based on Figures 4-11 - 4-19, we are able to come to several important conclusions:

1. In most cases, the difference between the approximate (green) and the corresponding exact (blue) curves are negligible compared with the error to the simulated curve. This leads us to the conclusion that the approximations that we have made are very good. Since they are significantly simpler than the exact forms, this is a very valuable finding. We note however, in Figures 4-17 and 4-18, where we considered UL - PC, that the exact BLN curves perform much better in the upper tail than their approximate counterparts. This is because, in the approximate expression, we assumed $R_{j}$ and $L_{j}$ to be independent. We conclude that this independence assumption should not be made, and that the modified method for the UL - PC case is the proper way to proceed.

2. For all cases where there is full activity $(p=1)$, we observe that the BPLN outperforms the BLN method, particularly in the lower tail. This is to be expected: moment-matching tends to match the higher values of the distribution [43], but the lower tail cannot be simultaneously matched, because the SLN $c d f$ bends on LN paper, whereas the BLN method cannot. However, since the BPLN was designed to match the slope of the lower tail of the SLN $c d f$, we observe that this indeed happens. When the BPLN does not match the lower tail well, it nevertheless runs parallel to it. We would need more degrees of freedom in the approximating $c d f$ to always match the tail, not only in slope, but also in offset.

3. For low activity $(p \leq 0.1)$, we observe that the BLN method performs very well, while the BPLN method does not fit the lower tail properly. In the case when $p N<1$, the BPLN curve is not even defined. Incidentally, notice that in the lower tail, the probability does 
not go to 0 , but tapers off to some value. This value is $P(I=0)=1-p_{\chi}$. We can observe that multiplying our LN and PLN models by a Bernoulli RV was a judicious move.

4. The conclusion is to use the BPLN method for $p$ close to 1 , and use the BLN method for $p$ for values around 0.1 and lower. For moderate values of $p$, the problem remains open, as neither method works well in the lower tail. It would seem that in this case more work is needed, analysing the problem of a sum of Bernoulli-times-lognormal RV's separately from the SLN problem. In the mean time, it would seem that the BLN and BPLN methods give two bounds for the lower tail. Perhaps a weighed average of the two approximating $c d f$ s, with weights that are functions of $p$, would give good results.

5. In general, leaving $p$ aside, the method works well for a wide variety of shadowing, fading and propagation constants, in both UL and DL, with and without PC. It works for many practical cellular layout scenarios, and seems to perform better for regular layouts than irregular (Figure 4-19) ones. It is not clear why this is the case.

6. Observing Figures 4-16 and 4-17, we see that the performance in the lower tail degrades as the fading model becomes more Rayleigh $(K \rightarrow 0)$. This could be anticipated from the results in Section 4.2.5.4, where we found that fading causes the SLN $c d f$ to bend significantly in the lower tail. However, we are pleased that the effect does not seem to be cumulative when we add several such RV. In fact, even with the unpromising results for one interferer, the approximation to the sum of multiple interferers' powers is quite good.

In conclusion, for low loading we suggest the BLN model, and for $p=1$, the BPLN model. Notice that the BPLN model dramatically outperforms the classical moment-matching approach for constant activity and is perhaps the best known quasi-closed-form solution to the problem. 


\section{Chapter 5 \\ Conclusion and Future Work}

We conclude with recalling the contributions that were made in this thesis, and propose several ideas for improving our method, and applying it to more complicated problems.

\subsection{Achievements of This Thesis}

While we did not meet all the ambitious requirements that we set up for ourselves in Section 1.1.3, we were nevertheless able to develop a set of quite simple equations that approximate the SLN distribution and the interference distribution with better precision than other known methods of comparable complexity.

\subsubsection{Tails of Sum of Independent Lognormals}

We showed that the lower tail of the SLN distribution in the independent case can be approximated by a LN distribution with parameters given by closed-form expressions. To our knowledge, there has to date been no analytical study of the lower tail. We defined the best lognormal fit in order to formally study whether the SLN $c d f$ has LN tails, and also whether proposed approximating $c d f$ 's have this property. We propose the $b l f$ to be a design criterion for future approximating $c d f$ 's. This will ensure that the approximation remains good in the far tails, and also that moment-matching can be used.

\subsubsection{Simple Expressions for Moments of Total Interference Power}

We gave exact closed-form expressions for the first two statistical moments of the total interference power in a cellular system, not only for UL, but also for the correlated DL case. These expressions contain geometrical coefficients that have been tabulated against the 
propagation constant and can be used directly in our expressions. We also showed that the expression for the second moment can be significantly simplified without loss of precision.

\subsubsection{Approximate Interference Distributions: Two Methods}

We have found that applying the FW method to the interference problem using a BLN resulted in simple closed-form expressions and gave excellent results for low probabilities of user activity $(p)$. For fully loaded systems with continuous interference $(p=1)$, we have found that using a PLN function to approximate the interference distribution also gave good results, particularly in the lower tail, which has often been neglected.

\subsection{Ideas for Continuation of this Work}

While working on the material presented in this thesis, we have encountered many other ideas that follow the same general line of reasoning, and that could potentially give an even better solution to the problems of the SLN and interference distributions. We also show that our approach can be modified to solve for a more detailed interference model without altering the fundamentals of our method.

Time did not permit us to follow all these ideas, so we only give a sketch of them here, with the hope that we, or someone else, will develop them more fully.

\subsubsection{Improving the SLN and Interference Approximations}

We have developed two methods for analysing the interference distribution: a moment-matching method using a BLN distribution, and a novel method using a BPLN distribution. We found that the second method performs very well when $p=1$, i.e., the distribution is PLN. The fit in the upper tail and body is always excellent, but tends to somewhat diverge in the lower tail. There are a few reasons for this: 
1. The distribution of the interference $I_{i}$ coming from just one cell is not quite well approximated in the lower tail, as seen in Figures 4-5 -4-10.

2. The PLN distribution does not have a blf in the lower tail, while the SLN distribution does. Only the slopes are matched in the lower tail, as can be seen in Figures 4-11 -4-19.

3. The lower tail was calculated based on the assumption that all summands are independent. However, in the DL, the individual interference components are slightly correlated.

The second reason could be remedied by developing a better SLN method, one that matches both tails' blf, while also fitting the body. We have proposed such a method in Sections 3.2.2.2 and 3.3.2. However the initial results show a very poor match in the body of the distribution in Figures 3-5-3-8. We believe that by introducing additional free parameters to the $c d f$ it would be possible to make the curve fit in the body while retaining the tail properties. The additional parameters could be solved by moment-matching. The moments of the SLN are known in closed form. However, the moments of the new approximating distribution will probably need to be found by numerical integration. However, this integration can probably be performed simply by using a Riemann sum on the transformed integral:

$$
E\left[X^{n}\right]=\int_{0}^{+\infty} x^{n} f(x) d x=\int_{-\infty}^{+\infty} e^{x(n+1)} f\left(e^{x}\right) d x
$$

Since $f(x)$ is approximately LN in each tail, $f\left(e^{x}\right)$ will be approximately Gaussian in each tail, i.e., it will decay as $e^{-x^{2}}$, and the integral can be truncated at moderate values in both limits. 


\subsubsection{Correlated Shadowing}

We have assumed that all wireless paths suffer from independent shadowing. However, the shadowing paths are in fact correlated, with correlation coefficients depending on distance and angular separation [61]. We will briefly show how our moment-matching method can be expanded to include the correlated shadowing case.

We begin with a set of correlated LN RV's $\left\{Y_{i}\right\}_{i=1}^{N}$ where $\left\{\ln Y_{i}\right\}_{i=1}^{N}$ form a set of jointly Gaussian RV's. Then we may then write:

$$
\left[\begin{array}{c}
\ln Y_{1} \\
\ln Y_{2} \\
\vdots \\
\ln Y_{N}
\end{array}\right]=\mathbf{M}\left[\begin{array}{c}
Z_{1} \\
Z_{2} \\
\vdots \\
Z_{N}
\end{array}\right]
$$

where $\mathbf{M}$ is a matrix and $\left\{Z_{i}\right\}_{i=1}^{N}$ are i.i.d. standard Gaussian RV's. The first moment of $X=\sum_{i=1}^{N} Y_{i}$ is not affected by the correlation and can be found from (2.17). The second moment can be calculated according to (2.18), where the correlation coefficient $\rho_{i, j} \neq 0$.

Now let us examine how we can incorporate this into our analysis of the interference moments. For simplicity, let us consider a system with two interferers, no fast fading $(K=\infty)$, constant activity $(p=1)$, and no PC $\left(P_{i}=1\right)$. This can be modeled by a simplified version of $(1.1)$ :

$$
I=R_{1} \zeta_{1}+R_{2} \zeta_{2}
$$

The second moment can then be calculated as 


$$
\begin{gathered}
E\left[I^{2}\right]=\left(E\left[R_{1}^{2}\right]+E\left[R_{2}^{2}\right]\right) E\left[\zeta_{1}^{2}\right]+2 E\left[R_{1} R_{2} \zeta_{1} \zeta_{2}\right] \\
=A_{2} e^{2 \sigma^{2}}+2 E\left[R_{1} R_{2} e^{\ln \zeta_{1}+\ln \zeta_{2}}\right] \\
=A_{2} e^{2 \sigma^{2}}+2 E\left[R_{1} R_{2} e^{\left(M_{11} Z_{1}+M_{12} Z_{2}\right)+\left(M_{21} Z_{1}+M_{22} Z_{2}\right)}\right] \\
=A_{2} e^{2 \sigma^{2}}+2 E\left[R_{1} R_{2} e^{\left(M_{11}+M_{21}\right) Z_{1}+\left(M_{12}+M_{22}\right) z_{2}}\right] \\
=A_{2} e^{2 \sigma^{2}}+2 E\left[R_{1} R_{2} e^{\left(M_{11}+M_{12}\right)\left(Z_{1}+Z_{2}\right)}\right] \\
=A_{2} e^{2 \sigma^{2}}+2 E\left[R_{1} R_{2} e^{\sqrt{2}\left(M_{11}+M_{12}\right) Z_{12}}\right] \\
=A_{2} e^{2 \sigma^{2}}+2 E\left[R_{1} R_{2} e^{\sqrt{2} M_{12} Z_{12}}\right] E\left[e^{\sqrt{2} M_{11} Z_{12}}\right] .
\end{gathered}
$$

Notice that, in this case, the matrix term M12 = M21 is itself a RV, correlated with R1 and R2, while M11 = M22 is related to the shadowing variance, and Z12 is an independent standard Gaussian RV.

Now in the case of a sum of correlated LN RV's, the behaviour in the upper tail will be the same as in the independent case [21], and so will the first moment. It is in the body and lower tail that there might be a substantial difference.

\subsubsection{More Realistic User Distribution}

We have assumed in this work that the MU connects to the FS that is physically nearest. This is not always a realistic assumption, as in most cellular systems the MU connects to the FS of which it receives the strongest long-term power. In this case, while the distribution of the location of the MU's remains spatially uniform, the distribution of the location of an MU (conditioned upon being) connected to a particular FS is no longer uniform within that cell's area. Because of this, the distribution functions of $R_{i}$ and $L_{i}$ are significantly different [62], and should be modified in our analysis. It is important to note that, in that case, both the pathloss and the shadowing combine together to decide to which FS an MU will connect, and the problem becomes more challenging because the distributions of $R_{i}$ and $L_{i}$ are a function of the shadowing variance $\sigma$. 


\subsubsection{Calculating the SIR, SINR and Outage Probability}

Our analysis has not included noise. This is because we have considered flat-fading systems, which use narrowband channels (OFDMA, FH-SS). Narrowband systems are generally more affected by interference than wideband systems [2]. Much of our analysis has focused on studying the lower tail of the interference $c d f$. In fact, this lower tail would be affected by adding a noise term. One could say that the analysis of the lower tail of the $c d f$ is then of little interest. However, the amount of noise added will vary from system to system, and it is not evident how much of the lower tail is really needed. This is why a good knowledge of the lower tail is still valuable. The calculation of the Signal-to-Interference Ratio (SIR) or Signal-to-Interference-andNoise Ratio (SINR) is a problem in itself:

1. In the case of DL, no PC, the signal pathloss affecting signal strength will be correlated with the interference because the position of the MU affects both signal and interference powers. New geometrical coefficients need to be devised.

2. We can model the signal strength as LN, due to the shadowing that the signal suffers, and the other effects (fading, pathloss) can be incorporated into this LN RV similarly to what was done in Section 4.1.

3. If the interference is modeled as a $\mathrm{LN}$ as in Section 4.4, the ratio of two LN RV's is simply a LN RV with log-variances added and log-means subtracted. The Bernoulli RV does not affect this calculation.

4. If the interference is modeled as a PLN as in section 4.5, the ratio of a LN and a PLN RV is not known. The integral involved in calculating the $c d f$ of that ratio is: 


$$
\int_{-\infty}^{+\infty} e^{B y-\frac{1}{2} A y^{2}} \Phi^{t-1}(y) d y
$$

where $A$ and $B$ are two constants. It is almost identical to the integral (3.50), which gives the moments of the PLN distribution. This makes the integral even more important to solve.

The outage probability in a system is simply found by calculating the probability that the SIR or SINR is above a certain threshold, predetermined by the modulation and coding scheme. The SIR/SINR is studied in [1], [3], [7]-[9], [12], [16], [59], [63].

\subsubsection{Application to Fixed Relay Networks}

We consider a two-hop fixed relaying scenario [2]. We define an augmented cell as the combination of a central sub-cell containing a BS and its surrounding six sub-cells containing RS's. The six RS's each have a good orthogonal wireless link with their BS. There is no communication between augmented cells, and we can assume that the interference from all sub-cells within the augmented cell has been adequately managed [2] and is negligible, while all other sub-cells are considered as interferers (see Figure 5-1).

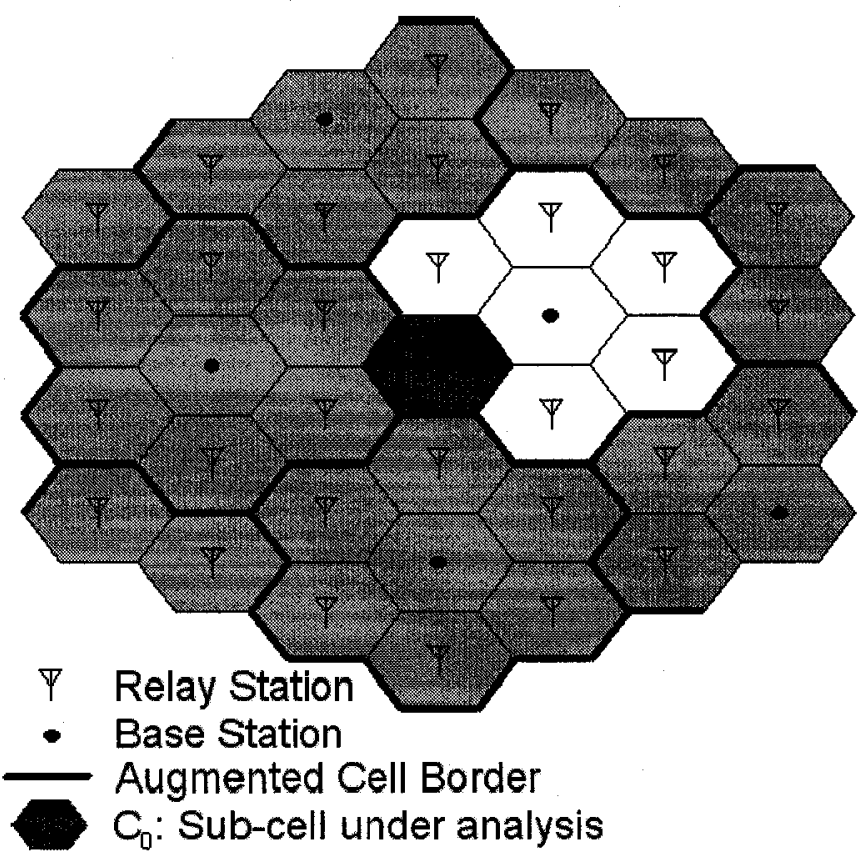

Figure 5-1 Interfering cells (in gray) in a two-hop fixed relay network. 


\subsubsection{Irregular Geometry}

As a first approximation to the problem, we can describe the set of interfering sub-cells as having the layout described by Figure 5-1, with geometry parameters given in Table 5-1.

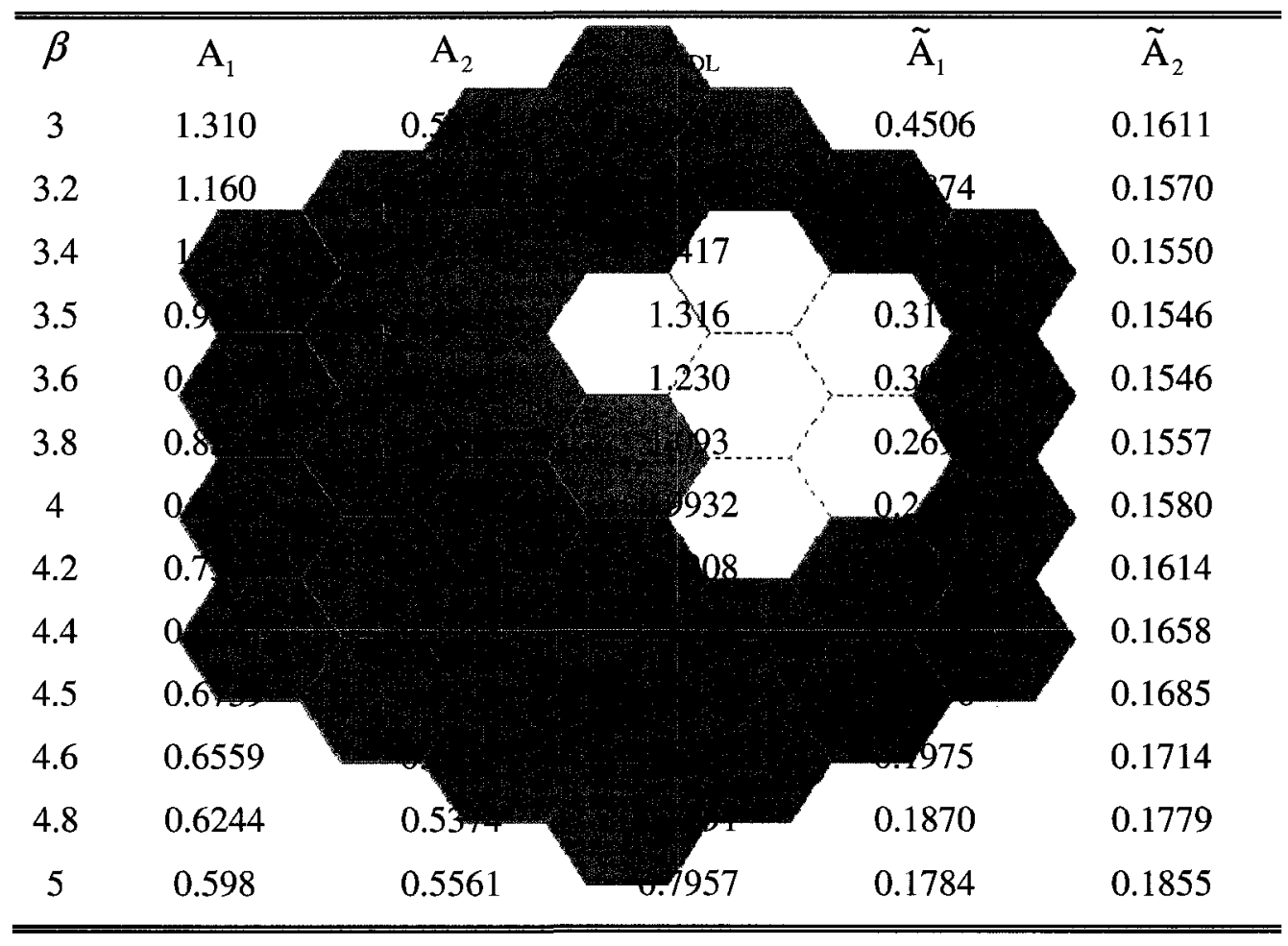

Table 5-1 Geometry parameters for three tiers of sub-cells in a two-hop fixed relay network.

\subsubsection{Lower Probability of Activity per Sub-cell}

It can be argued that the probability of activity within any sub-cell will be substantially lower than 1. Indeed, [2] defines loading for such a network to be 1 when there is one user per channel per augmented cell. This corresponds to $p \approx 0.14$. A more aggressive reuse scheme can be used, where each channel is used once per sub-cell, which corresponds to a loading of 7 and $p=1$.

In the approach proposed in this section, we have assumed that there is no interference coming from within the augmented cell. This corresponds to a scenario where the loading is 1 or less, and 
is only approximately true for higher loading. Thus we will be primarily interested in values of $p$ in the range $[0,0.14]$, where our methods perform well, as will be seen in the following section.

\subsubsection{Some Simulation Results}

We simulate the interference in the fixed relay network in the same way as we did in Section 4.6, except that the layout is different. The simulation parameters are summarized in Table 5-2, and the results are shown in Figure 5-2.

\begin{tabular}{|c|c|c|c|c|}
\hline Figure & \multicolumn{4}{|c|}{$5-2$} \\
\hline$\beta$ & \multicolumn{4}{|c|}{4} \\
\hline$\sigma[\mathrm{dB}]$ & \multicolumn{4}{|c|}{12} \\
\hline $\boldsymbol{K}$ & \multicolumn{4}{|c|}{0} \\
\hline$p$ & 0.035 & 0.14 & 0.5 & 1 \\
\hline Layout & \multicolumn{4}{|c|}{ 3-Tier Two-Hop Relay } \\
\hline Link & \multicolumn{4}{|c|}{ DL } \\
\hline $\mathbf{P C}$ & \multicolumn{4}{|c|}{ Yes $\left(\sigma_{e}=1 \mathrm{~dB}\right)$} \\
\hline$p_{\chi}$ & 0.6566 & 0.9892 & $1-9.3 \cdot 10^{-10}$ & 1 \\
\hline$a_{I}=$ & -6.601 & -5.136 & -3.243 & -2.203 \\
\hline$a_{I} \approx$ & -6.601 & -5.136 & -3.243 & -2.203 \\
\hline$b_{1}=$ & 4.394 & 4.281 & 4.131 & 4.046 \\
\hline$b_{1} \approx$ & 4.353 & 4.239 & 4.088 & 4.002 \\
\hline$m_{1}=$ & -5.976 & -6.38098 & -6.38056 & -6.369 \\
\hline$m_{I} \approx$ & -5.921 & -6.32710 & -6.32711 & -6.316 \\
\hline$s_{I}$ & \multicolumn{4}{|c|}{4.225} \\
\hline$t_{I}=$ & 1.108 & 4.434 & 15.83 & 31.67 \\
\hline$t_{I} \approx$ & 1.05 & 4.2 & 15 & 30 \\
\hline
\end{tabular}

Table 5-2 Simulation and analytical parameters for a two-hop fixed relay network. 


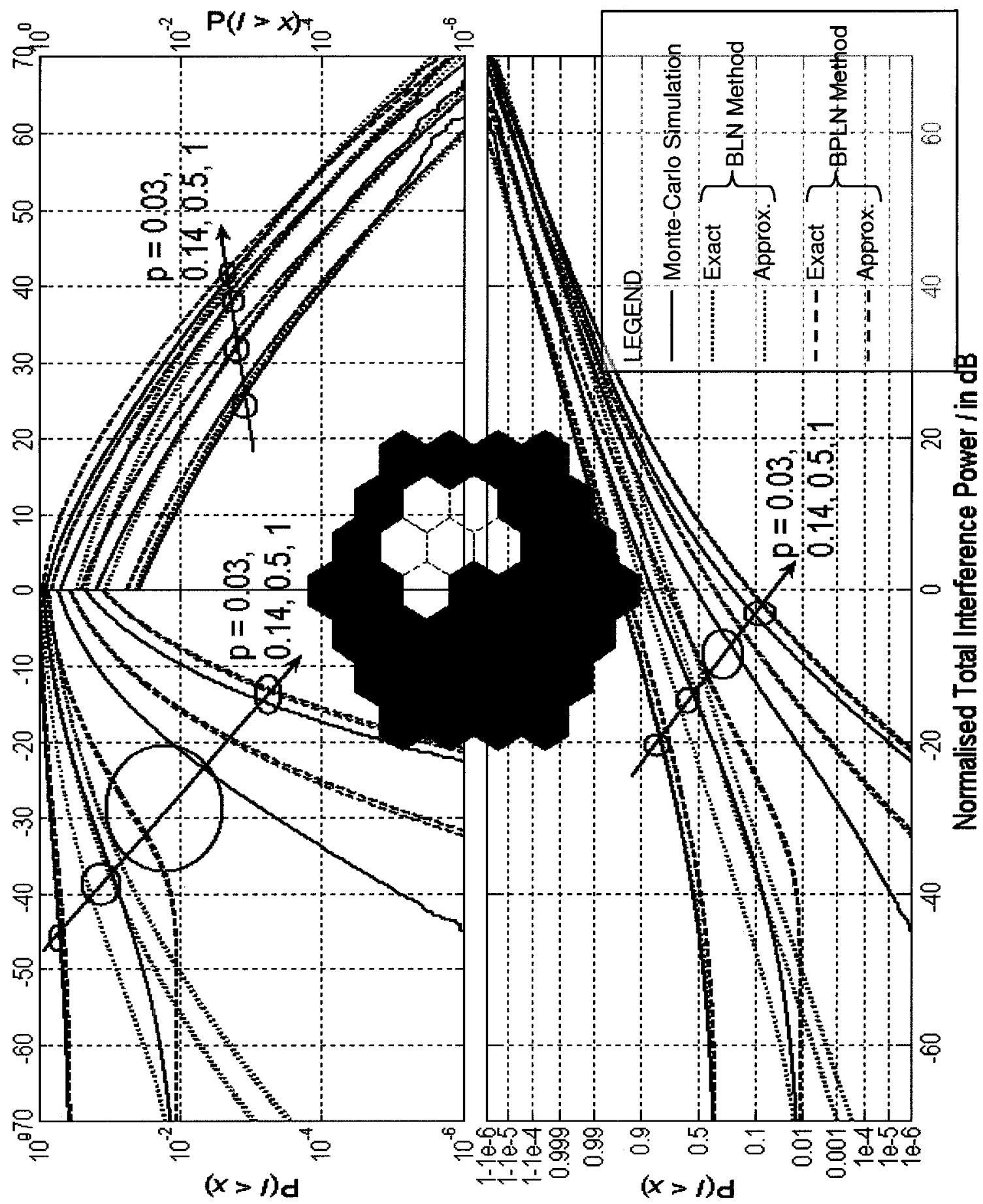

Figure 5-2 Interference from a fixed relay network in the downlink with power control. 


\subsubsection{Evolution of Interference in Time}

Throughout this work, we have investigated what is the distribution of the total interference power at any given instant. However, in order to get useful knowledge about such performance statistics as word or packet error rates, quality of service, etc., it is important to understand how the total interference power evolves in time, which would require studying the interference as a random process. This has already been done in various ways in literature [11]-[13]. We hope to expand our method to include the evolution of interference in time, so as to obtain very precise long-term performance statistics.

\subsubsection{CDMA Systems}

It would be interesting to apply our method to broadband CDMA networks; however there are several necessary considerations:

1. The channel is no longer flat-fading.

2. The number of users in each cell is no longer one, but can be quite large. This has at least two important consequences:

3. The number of summands in the SLN method needs to be large. Large values of $N$ have not typically been analysed. We are only aware of the LL method proving robust for as much as 1000 summands (Figure 6 in [27]). Incidentally, our PLN method does not perform very well for $N=1000$ (we do not show the simulations here).

4. The correlation between shadowing paths needs to be studied. In the UL, many users will be located within the same narrow angular sector, which implies high shadowing correlation [61]. The independence assumption will no longer be acceptable. 


\section{Bibliography}

[1] Z. Kostic, I. Maric, and X. Wang, "Fundamentals of dynamic frequency hopping in cellular systems," IEEE Journal on Selected Areas in Communications, vol. 19, pp. 2254-2266, November 2001.

[2] O. Mubarek, H. Yanikomeroglu, and S. Periyalwar, "Dynamic frequency hopping in cellular fixed relay networks," IEEE Vehicular Technology Conference, Spring 2005, vol. 5, pp. 3112-3116, May 2005.

[3] M. Chiani, A. Conti, and O. Andrisano, "Outage evaluation for slow frequency-hopping mobile radio systems," IEEE Transactions on Communications, vol. 47, pp. 1865-1874, December 1999.

[4] M. Pratesi, F. Santiccu, and F. Graziosi, "Generalized moment matching for the linear combination of lognormal RVs: application to outage analysis in wireless systems," IEEE Transactions on Wireless Communications, vol. 5, pp. 1122-1132, May 2006.

[5] T. Liu, D. Everitt, “Analytical approximation of other-cell interference in the uplink of CDMA cellular systems," IEEE Vehicular Technology Conference, Spring 2006, vol. 2, pp. 693-697, 2006.

[6] R. Prasad and A. Kegel, "Improved assessment of interference limits in cellular radio performance," IEEE Transactions on Vehicular Technology, vol. 40, pp. 412-419, May 1991.

[7] C.-C. Lee and R. Steele, "Signal-to-interference calculations for modern TDMA cellular communication systems," IEE Proceedings on Communications, vol. 142, pp. 21-30, February 1995. 
[8] A. Annamalai, C. Tellambura, and V.K. Bhargava, "Simple and accurate methods for outage analysis in cellular mobile radio systems - a unified approach," IEEE Transactions on Communications, vol. 49, pp. 303-316, February 2001.

[9] M. Pratesi, F. Santucci, F. Graziosi, and M. Ruggieri, "Outage analysis in mobile radio systems with generically correlated log-normal interferers," IEEE Transactions on Communications, vol. 48, pp. 381-385, March 2000.

[10] M.-S. Kwok and H.-S. Wang, "Adjacent cell interference analysis of reverse-link in CDMA cellular radio systems," IEEE International Symposium on Personal, Indoor and Mobile Radio Communications, PIMRC'95, vol. 2, pp. 446-450, September 1995.

[11] K. Navaie, S. Valaee, A. R. Sharafat, and E. S. Sousa, "On the downlink interference in heterogeneous wireless DS-CDMA networks," IEEE Transactions on Wireless Communications, vol. 5, pp. 384-393, February 2006.

[12] F. Graziosi, L. Fuciarelli, and E. Santucci, "Second order statistics of the SIR for cellular mobile networks in the presence of correlated co-channel interferers," IEEE Vehicular Technology Conference Spring 2001, vol. 4, pp. 2499-2503, May 2001.

[13] C. Fischione, F. Graziosi, and F. Santucci, "Approximation for a sum of on-off log-normal processes with wireless applications," IEEE International Conference on Communications, vol. 1, pp. 306-310, June 2004.

[14] L.-C. Wang and C.-T. Lea, "Co-channel interference analysis of shadowed Rician channels," IEEE Communications Letters, vol. 2, pp. 67-69, March 1998. 
[15] Q. T. Zhang, “Co-channel interference analysis for mobile radio suffering lognormal shadowed Nakagami fading," IEE Proceedings on Communications, vol. 146, pp. 49-54 February 1999.

[16] M. Pratesi, F. Santucci, and F. Graziosi, "Generalized moment-matching for the linear combination of lognormal RVs - application to outage analysis in wireless systems," The $17^{\text {th }}$ Annual IEEE International Symposium on Personal, Indoor and Mobile Radio Communications (PIMRC'06), pp. 1 - 5, September 2006.

[17] H. Chang and S. S. Sapatnekar, "Full-chip analysis of leakage power under process variations, including spatial correlations," 42nd Annual ACM IEEE Design Automation Conference, pp. 523-528, 2005

[18] M. Romeo, V. Da Costa, and F. Bardou, "Broad distribution effects in sums of lognormal random variables," The European Physical Journal B, vol. 32, pp. 513-525, 2003.

[19] R. Barakat, "Sums of independent lognormally distributed random variables," Journal of the Optical Society of America, vol. 66, pp. 211-216, 1976.

[20] M. Milevsky and S. Posner, "Asian options, the sum of lognormals and the reciprocal Gamma distribution," Journal of Financial and Quantitative Analysis, vol. 33, pp. 409-422, 1998.

[21] S. Asmussen and L. Rojas-Nandayapa, "Sums of dependent lognormal random variables: asymptotics and simulation", December 2005.

[22] R. B. Leipnik, “On lognormal random variables: I-the characteristic function," Journal of the Australian Mathematical Society Ser. B, vol. 32, pp. 327-347, 1991 
[23] E. M. Healy and T. J. Young, "Determining traffic asymmetry of future mobile services," Telecompetition, Inc., March 2004.

[24] A. Iyer, C. Rosenberg, and A. Karnik, "What is the Right Model for Wireless Channel Interference?," The Third International Conference on Quality of Service in Heterogeneous Wired/Wireless Networks (QShine 2006), Waterloo, Canada, August 2006.

[25] J. Lin and W. Wu, "Performance analysis of LDPC on Rician fading channels," Front. Electr. Electron. Eng. China, vol. 1, pp. 120-124, 2006, Translated from Chinese from Journal of Beijing University of Posts and Telecommunications, vol. 27, pp. 48-53, 2004.

[26] L. Fenton, "The sum of log-normal probability distributions in scatter transmission systems," IEEE Transactions on Communications, vol. 8, pp. 57-67, March 1960.

[27] C. L. J. Lam and T. Le-Ngoc, "Log shifted gamma approximation to lognormal sum distributions," IEEE ICC 2005, vol. 1, pp. 495-499, May 2005.

[28] Q. T. Zhang and S. H. Song, "Model Selection and Estimation for Lognormal Sums in Pearson's Framework," IEEE Vehicular Technology Conference Spring 2006, vol. 6, pp. 2823-2827, 2006.

[29] L. Zhao and J. Ding, “A strict approach to approximating lognormal sum distributions", IEEE CCECE 2006, pp.286-289, May 2006.

[30] N. C. Beaulieu and F. Rajwani, "Highly accurate simple closed-form approximations to lognormal sum distributions and densities," IEEE Communications Letters, vol.8, pp. 709_ 711, December 2004.

[31] K.-I. Sato, “Convolution of unimodal distributions can produce any number of modes," The Annals of Probability, vol. 21, pp. 1543-1549, 1993. 
[32] C.-L. J. Lam and T. Le-Ngoc, "Estimation of typical sum of lognormal random variables using log shifted Gamma approximation", IEEE Communications Letters, vol. 10, pp. 234235, April 2006.

[33] K. Sigman, “Appendix: A primer on heavy-tailed distributions," Queueing Systems, vol. 33, pp. 261-275, 1999.

[34] J. L. Geluk and C. G. De Vries, "Weighted sums of subexponential random variables and asymptotic dependence between returns on reinsurance equities," submitted, July 2004.

[35] N. C. Beaulieu and Q. Xie, "An optimal lognormal approximation to lognormal sum distributions," IEEE Transactions on Vehicular Technology, vol. 53, pp. 479-489, March 2004.

[36] J. Wu, N. B. Mehta, and J. Zhang, "Flexible lognormal sum approximation method," IEEE GLOBECOM 2005, vol. 6, pp. 3413-3417, November 2005.

[37] S. C. Schwartz and Y. S. Yeh, "On the distribution function and moments of power sums with lognormal components," Bell System Technical Journal, vol. 61, pp. 1441-1462, 1982.

[38] P. Cardieri and T. S. Rappaport, "Statistics of the sum of lognormal variables in wireless communications," IEEE Vehicular Technology Conference Spring 2000, vol. 3, pp. 18231827, May 2000.

[39] D. Schleher, "Generalized Gram-Charlier series with application to the sum of log-normal variates," IEEE Transactions on Information Theory, vol. 23, pp. 275-280, March 1977.

[40] J. C. S. Santos Filho, M. D. Yacoub, and P. Cardieri, "Highly accurate range-adaptive lognormal approximation to lognormal sum distributions," IEE Electronics Letters, vol. 42, pp. 361-362, March 2006. 
[41] F. Berggren, “An error bound for moment matching methods of lognormal sum distributions," March 2005.

[42] W. A. Janos, "Tail of the distribution of sums of lognormal variates," IEEE Transactions on Information Theory, vol. IT-16, pp. 299-302, May 1970.

[43] B. G. Lindsay and P. Basak, "Moments determine the tail of a distribution (but not much else)," The American Statistician, vol. 54, November 2004.

[44] S. Ben Slimane, "Bounds on the distribution of a sum of independent lognormal random variables," IEEE Transactions on Communications, vol. 49, pp. 975-978, June 2001.

[45] N. C. Beaulieu, A. A. Abu-Dayya, and P. J. McLane, "Comparison of methods of computing lognormal sum distributions and outages for digital wireless applications," IEEE International Conference on Communications ICC'94, vol. 3, pp. 1270-1275, May 1994.

[46] F. Berggren and S. Ben Slimane, "A simple bound on the outage probability with lognormally distributed interferers," IEEE Communications Letters, vol. 8, pp. 271-273, May 2004.

[47] Y. S. Yeh and S. C. Schwartz, "Outage Probability in Mobile Telephony Due to Multiple Log-Normal Interferers," IEEE Transactions on Communications, vol. 32, pp. 380-388, April 1984.

[48] P. Pirinen, "Statistical power sum analysis for nonidentically distributed correlated lognormal signals," The 2003 Finnish Signal Processing Symposium (FINSIG'03), pp. 254258, Tampere, Finland, May 2003. 
[49] A. Safak, "Statistical analysis of the power sum of multiple correlated log-normal components," IEEE Transactions on Vehicular Technology, vol. 42, pp. 58-61, February 1993.

[50] C. L. J. Lam and T. Le-Ngoc, "Outage probability with correlated lognormal interferers using log shifted gamma approximation," Fifth International Conference on Information, Communications and Signal Processing, pp. 618-622, December 2005.

[51] N. B. Mehta, A. F. Molischm, J. Wu, and J. Zhang, "Approximating the sum of correlated lognormal or, lognormal-Rice random variables," IEEE International Conference on Communications 2006, vol. 4, pp. 1605-1610, June 2006.

[52] J. A. Gubner, "A new formula for lognormal characteristic functions," IEEE Transactions on Vehicular Technology, vol. 55, pp. 1668-1671, September 2006.

[53] N. C. Beaulieu, "A simple integral form of lognormal characteristic functions convenient for numerical computation", IEEE Globecom 2006.

[54] J. C. S. Santos Filho, P. Cardieri, and M. D. Yacoub, "Simple Accurate Lognormal Approximation to Lognormal Sums," IEE Electronics Letters, vol. 41, pp. 1016-1017, September 2005.

[55] M. A. Lifshits, "On the lower tail probabilities of some random series," The Annals of Probability, vol. 25, pp. 424-442, 1997.

[56] N. M. Temme, "Uniform asymptotic expansions of the incomplete gamma functions and the incomplete beta function," Mathematics of Computation, vol. 29, pp. 1109-1114, October 1975. 
[57] H. E. Fettis, "A stable algorithm for computing the inverse error function in the "tailend" region," Mathematics of Computation, vol. 28, pp. 585-587, April 1974.

[58] W. Nelson and N. Doganaksoy, "A computer program POWNOR for fitting the PowerNormal and -Lognormal Models to life or strength data from specimens of various sizes," U.S. Department of Commerce, Technology Administration, National Institute of Standards and Technology, Center for Computing and Applied Mathematics, Statistical Engineering Division, March 1992.

[59] J. Sijbers, A. J. den Dekker, P. Scheunders, and D. Van Dyck, "Maximum-likelihood estimation of Rician distribution parameters," IEEE Transactions on Medical Imaging, vol. 17, pp. 357-361, June 1998.

[60] G. Rempała and J. Wesołowski, "Asymptotics for products of sums and $U$-statistics," Electronic Communications in Probability, vol. 7, pp. 47-54, 2002.

[61] Thomas Klingenbrunn and Preben Mogensen, "Modelling cross-correlated shadowing in network simulations," IEEE Vehicular Technology Conference Fall 1999, vol. 3, pp. 14071411, September 1999.

[62] V. M. Jovanovic and J. Gazzola, "Capacity of present narrowband cellular systems: interference-limited or blocking-limited?," IEEE Personal Communications, vol. 4, pp. 42 51, December 1997.

[63] A. Persson, T. Ottosson, and E. G. Strom, "Analysis of the downlink BER performance for coded OFDMA with fading co-channel interference," 2004. 


\section{Papers that have resulted from our thesis work:}

[64] S. S. Szyszkowicz, H. Yanikomeroglu, E. Fituri, and S. Periyalwar, "Analytical modeling of interference in cellular fixed relay networks," IEEE CCECE 2006, pp. 376-379, May 2006

[65] S. S. Szyszkowicz and H. Yanikomeroglu, "On the tails of the distribution of lognormal sums," IEEE ICC 2007, under review.

These last two papers can be obtained from our website: www.sce.carleton.ca/ sz . 